\title{
Associations of occupational standing with musculoskeletal symptoms - a systematic review with meta-analysis
}

\section{Authors}

Pieter Coenen

School of Physiotherapy and Exercise Science, Curtin University, Perth, Australia

\section{Lisa Willenberg}

Centre for International Health, Burnet Institute, Melbourne, Australia

Sharon Parry

School of Physiotherapy and Exercise Science, Curtin University, Perth, Australia.

Joyce W Shi

Monash Health, Melbourne, Victoria, Australia

Lorena Romero

Alfred Hospital, Melbourne, Australia

Diana M Blackwood

Faculty of Health Sciences, Curtin University, Perth, Australia

\section{Christopher Maher}

Musculoskeletal Division, The George Institute for Global Health, Sydney Medical School, The University of Sydney, Sydney, Australia

\section{Genevieve N Healy}

The University of Queensland, School of Public Health, Brisbane, Australia

Baker IDI Heart and Diabetes Institute, Melbourne, Australia;

School of Physiotherapy and Exercise Science, Curtin University, Perth, Australia.

\section{David W Dunstan}

The University of Queensland, School of Public Health, Brisbane, Australia

Baker IDI Heart and Diabetes Institute, Melbourne, Australia;

School of Public Health and Preventive Medicine, Monash University, Melbourne, Australia

School of Exercise and Nutrition Sciences, Deakin University, Geelong, Australia

Department of Medicine, Monash University, Melbourne, Australia

School of Sport Science, Exercise and Health, The University of Western Australia, Perth, Australia

Mary MacKillop Institute for Health Research, Australian Catholic University, Melbourne, Victoria, Australia

Leon M Straker

School of Physiotherapy and Exercise Science, Curtin University, Perth, Australia.

\section{Word count}

Abstract: 245

Manuscript: 3965

Number of figures: 2

Number of tables: 2

Number of appendices: 14

No. of references: 79 


\section{ABSTRACT}

Objective: Given the high exposure to occupational standing in specific occupations, and recent initiatives to encourage intermittent standing among white-collar workers, a better understanding of the potential health consequences of occupational standing is required. We aimed to review and quantify the epidemiological evidence on associations of occupational standing with musculoskeletal symptoms.

Design: A systematic review was performed. Data from included articles were extracted and described, and meta-analyses conducted when data were sufficiently homogenous.

Data sources: Electronic databases were systematically searched (up to February 2015) Eligibility criteria: Peer-reviewed articles on occupational standing and musculoskeletal symptoms from epidemiological studies were identified.

Results: Of the 11,750 articles screened, 50 articles reporting 49 studies were included (45 cross-sectional and 5 longitudinal; n=88,158 participants) describing the associations of occupational standing with musculoskeletal symptoms, including low-back (39 articles), lower extremity (14 articles) and upper extremity (18 articles) symptoms. In the metaanalysis, 'excessive' (>4 hours/workday) occupational standing was associated with the occurrence of low-back symptoms (pooled odds ratio [95\% Cl] 1.31[1.10 1.56]). Evidence on lower and upper extremity symptoms was too heterogeneous for meta-analyses. The majority of included studies reported statistically significant detrimental associations of occupational standing with lower extremity, but not with upper extremity symptoms. Conclusions: The evidence suggests that 'excessive' occupational standing is associated with the occurrence of low-back and (inconclusively) lower extremity symptoms, but there may not be such an association with upper extremity symptoms. Only limited evidence from high quality, longitudinal studies using objectively measured standing was found. 
Key words: Musculoskeletal symptoms - occupational standing - systematic review - meta analysis

\section{WHAT IS ALREADY KNOWN:}

- There are high exposures to occupational standing in specific occupations and recent initiatives encouraging intermittent standing among white-collar workers.

- In light of these (shifting) working styles, some concerns have been expressed that occupational standing may expose workers to risks of health consequences such as musculoskeletal symptoms.

- The association of occupational standing with musculoskeletal symptoms is yet unclear, with no current systematic review quantifying this association.

\section{WHAT ARE THE NEW FINDINGS:}

- We systematically reviewed literature and identified substantial evidence (50 articles from $n=88,158$ participants) describing associations of occupational standing with low-back, lower extremity and upper extremity symptoms.

- We found evidence (including pooled data from a meta-analysis) for associations between occupational standing and the occurrence of low-back and (inconclusively) lower extremity symptoms, however not for upper extremity symptoms.

- We found only limited evidence from high quality, longitudinal studies using objectively measured standing was found.

- Such information is needed to provide more definitive evidence to inform good work design for both blue- and white-collar workers. 


\section{INTRODUCTION}

Exposure to extended periods of occupational standing is traditionally common among specific occupational groups, such as in the retail, food, healthcare, education, and manufacturing industries. For example, in a population of Australian workers, $62 \%$ reported working in a job that usually involved standing[1]; a finding consistent with that observed in a study conducted with a Canadian working population[2]. In a study with objectively measured standing (using thigh and hip worn accelerometers), it was shown that Danish blue-collar workers stood on average 2.2 (SD 1.3) hours per workday with subgroups standing for up to $3.7(0.7)$ hours per workday[3].

A recent and growing body of evidence suggests that excessive sitting is associated with several adverse health outcomes including poor cardio-metabolic health and premature mortality[4-6]. Consequently, there is a growing interest in workplace initiatives to reduce the amount of sitting time for sedentary workers[7], with expert recomendations advising workers to replace some sitting periods with standing and light activity at work[8], specifically by advocating regular postural changes (i.e. shifting between sitting, standing and moving). Replacing sitting with alternatives such as standing has been shown to be feasible[8 9] and is rapidly being implemented in many workplaces, such as through the introduction of sit/stand office workstations[10]. However, some concerns have been expressed that alternatives to sitting, such as standing, may expose workers to new hazards and/or other health consequences[11].

In light of shifting working styles, a sound understanding of the health consequences of occupational standing is urgently needed to inform healthy work practice for both blueand white-collar workers. Adverse health outcomes of standing have been reported 
before[12], with standing being associated with venous disorders of the lower extremities[13 14], perinatal health complications such as pre-term delivery and preeclampsia[15], and musculoskeletal symptoms such as low-back and lower limb symptoms[16 17]. Despite this body of evidence, the association of occupational standing with musculoskeletal symptoms (e.g. self-reported pain, discomfort or complaints in regions of the musculoskeletal system) is yet unclear, with no current systematic review quantifying this association. The aims of this study were to systematically review the epidemiological evidence on the associations of occupational standing with non-specific musculoskeletal symptoms, and to quantify this association by means of meta-analyses.

\section{METHODS}

\section{Search strategy}

To identify relevant publications, we performed systematic searches of the literature in the following bibliographic databases: Health \& Safety Science Abstracts (Proquest); CINAHL (EBSCO); EBM Reviews - Cochrane Central Register of Controlled Trials (Ovid); Embase (Ovid); Medline (Ovid); Psyclnfo (Ovid). Searches were performed from database inception to 10 February 2015 with search terms including controlled key terms as well as free text terms. Search terms expressing 'standing' were used in combination with search terms for 'work-related' (Appendix 1-6). No specific terms for 'health outcomes' were used as this study is part of larger review aimed at assessing the association of occupational standing and multiple health outcomes.

Two reviewers independently screened all potentially relevant titles and abstracts for eligibility. If necessary, full-text articles were checked for eligibility. Differences in judgment were resolved through a consensus procedure. Studies were included if they met the 
following criteria: the article was an original epidemiological study published in a peerreviewed journal (i.e., excluding reviews, editorials or letters, theses and conference proceedings); it was published in English; and it reported on the association between occupational standing and any health problem. Only articles that described a general adult working population were included (e.g., studies selecting workers with chronic disorders and non-adult populations were excluded). Only studies quantifying the association of occupational standing with health outcomes were included (thereby excluding qualitative research). Studies in which occupational standing was not a main exposure variable of interest (e.g., standing was only part of a certain condition/trial such as 'lifting during standing'), or in which occupational standing was only used as a confounding variable, were excluded.

All eligible full-text articles were classified on exposure (work-related vs non-work related), outcome (musculoskeletal or other outcomes) and study design (e.g., laboratory study, cross-sectional study or longitudinal study). For the current study, only articles describing epidemiological cross-sectional (case-control or cross-sectional observational studies) or longitudinal observational studies on occupational standing and their association with musculoskeletal symptoms (i.e., self-reported pain, discomfort and/or complaints in any region of the musculoskeletal system) were included. Full-text versions of the selected articles were obtained for data extraction and quality assessment. In cases where full-text articles could not be found through online and/or offline databases, authors were contacted. Reference lists of selected articles were screened to identify additional potentially eligible articles.

Data extraction and quality assessment 
Two reviewers independently assessed all selected articles for methodological quality and data extraction. In cases of disagreement, consensus was reached during a meeting. Methodological quality was evaluated using an adapted version of a published scoring system $^{[18]}$, based on eleven criteria for the reporting of study methods (description of recruitment, participants, allocation, measures, sample size) and results (description of variance, confounding, detail of results), with answer categories being 'yes', 'partial', 'no' and 'not applicable' (Appendix 7). Summary scores (ranging from 0 to 1 ) were calculated with:

Summary score $=\Sigma[($ number of 'yes' $\times 2)+($ number of 'partial' $\times 1)] / \Sigma[22-($ number of ' $\left.\left.N / A^{\prime}\right) \times 2\right]$

Studies with a summary score $\geq 0.75$ were considered to be of high methodological quality[18].

For data extraction, the following data from each included article were obtained: first author and year of publication, study name, study design, sample description (i.e., number of participants, age, gender, occupation and country), confounders, exposure (assessment and operationalisation of occupational standing), outcome (assessment and operationalisation of musculoskeletal symptoms) and exposure-outcome estimates (e.g., odds ratios [ORs], relative risks [RR]).

\section{Data-analysis}

All included studies were described according to their methodological quality and extracted data. Included articles were categorised into body areas regarding their outcomes; i.e., lowback symptoms, lower extremity symptoms, upper extremity symptoms and symptoms in any body area. 
In each of the four body areas, quantitative analyses of homogenous studies (with sufficient overlap in definitions of exposure, outcome, study population and study design for which small differences in definition of exposure and outcome were accepted[19]) were performed if possible. To be able to combine information from different studies, occupational standing was treated as a dichotomous variable for which a cut-off value of 4 hours/workday was adopted (i.e., durations of standing below this threshold were considered 'not excessive' while durations of standing above this threshold were considered 'excessive' standing). In the absence of any known 'threshold' for excessive standing, we selected this cut-off value based on the data provided in the identified studies with 4 hours/workday being the most often reported (with sensitivity analysis examining other thresholds). The cut-off is also consistent with the recent recommendations for those occupations which are predominately desk-based whereby the eventual progression to a total accumulation of up to 4 hours/workday is advocated to offset the health hazards associated with excessive sitting[8]. Model parameters (i.e., unadjusted ORs; or crosstabulations of participants exposed to not excessive and excessive standing, with and without musculoskeletal symptoms) were retrieved from the original studies. If needed, exposure categories from studies reporting multiple occupational standing categories were collated. In instances where data provided in the published articles were insufficient, corresponding authors were contacted and asked for additional information.

Review Manager (RevMan) version 5.3 was used to conduct meta-analyses and generate forest plots, using a random-effects model due to the heterogeneity of the studies. We report on ORs with $95 \%$ confidence intervals $(\mathrm{Cl})$ depicting each individual study as well as pooled exposure-outcome associations of excessive occupational standing and musculoskeletal symptoms, unadjusted for confounders. P-values $<0.05$ (two-sided) were 
considered statistically significant. Heterogeneity was assessed using $l^{2}$ statistics and visual inspection of the forest plots were performed, while subgroup analyses were conducted using $\chi^{2}$ statistics. Funnel plots were generated to assess publication bias (through visual inspection).

Sensitivity analyses were conducted to test the robustness of pooled-exposure associations. The sensitivity of the cut-off value for excessive occupational standing (4 hours/workday) was tested by comparing those studies for which we could estimate exposure-outcome associations with a 4 hours/workday cut-off value to those for which we could estimate exposure-outcome associations with a 2 hours/workday cut-off value (performing subgroup analysis). Due to a lack of sufficient homogeneous data, we were not able to test for the effect of other cut-off values. In a second sensitivity analysis, we compared exposure-outcome associations unadjusted for confounders to exposure-outcome associations adjusted for confounders (e.g., gender, age, other physical or mental work demands). We also tested for differences in exposure-outcome associations of studies reporting on generic samples of workers (i.e., random samples of a general working population or samples of mixed occupational groups) compared to studies on samples of specific occupational groups (e.g., only hospital staff, only construction workers). In a final sensitivity analysis we tested for differences in exposure-outcome associations of studies with low compared to high methodological quality.

\section{RESULTS}

\section{Data-description}

The flow chart of the search and selection process is presented in Figure 1. Our search strategy yielded a total of 15,857 search hits. After removing duplicates, 11,750 individual 
articles remained that were screened on their titles for inclusion. After excluding 10,951 records, a total of 799 abstracts were screened after which 356 abstracts did not meet the inclusion criteria. A total of 11 full-text articles could not be retrieved (even after contacting corresponding authors), providing a total of 432 full-text articles that were screened on eligibility, of which 218 met the criteria of describing outcomes of occupational standing. A total of 44 of these articles specifically addressed the association of occupational standing and musculoskeletal symptoms using an epidemiological study design. After screening the reference lists of these articles, six more articles were added, resulting in a final total of 50 articles (reporting 49 studies) included in the current review and used for methodological quality assessment and data-extraction (see Table 1 for a summary of findings)[17 20-68]. 
Table 1. Summary of findings from all identified evidence describing the number of studies ( $N$ ) and number of participants ( $n$ ) in each of the outcome (body area), study design, exposure assessment and study findings by categories.

\begin{tabular}{|c|c|c|c|c|c|c|c|c|c|c|c|c|c|}
\hline & & \multirow[t]{2}{*}{ Total } & \multicolumn{3}{|c|}{ Study design } & \multicolumn{3}{|c|}{ Exposure assessment } & \multicolumn{5}{|c|}{ Study findings } \\
\hline & & & $\begin{array}{c}\text { Cross- } \\
\text { sectional } \\
\text { case- } \\
\text { control }\end{array}$ & $\begin{array}{l}\text { Cross-sectional } \\
\text { observational }\end{array}$ & $\begin{array}{l}\text { Longitudinal } \\
\text { observational }\end{array}$ & $\begin{array}{l}\text { Self- } \\
\text { reports }\end{array}$ & Observations & Objective & $\begin{array}{c}\text { Meta- } \\
\text { analysis }\end{array}$ & $\begin{array}{c}\text { Positive }^{1} \\
\text { (significant) }\end{array}$ & $\begin{array}{c}\text { Negative }^{2} \\
\text { (significant) }\end{array}$ & $\begin{array}{c}\text { Non- } \\
\text { significant }\end{array}$ & $\begin{array}{l}\text { No data } \\
\text { reported }\end{array}$ \\
\hline \multirow[t]{2}{*}{ Low-back } & $\mathrm{N}$ & 39 & 4 & 31 & 4 & 37 & 1 & 1 & $16^{3}$ & 9 & - & 8 & 6 \\
\hline & $\mathrm{n}$ & 82,291 & 9,210 & 6,364 & 9,435 & 81,863 & 401 & 27 & $54,392^{3}$ & 9,239 & - & 7,330 & 11,330 \\
\hline \multirow[t]{2}{*}{ Lower extremity } & $\mathrm{N}$ & 14 & 3 & 9 & 2 & 13 & 1 & - & - & 8 & 1 & 4 & 4 \\
\hline & $\mathrm{n}$ & 31,924 & 5,081 & 24,147 & 2,696 & 31,903 & 21 & - & - & 24,502 & 759 & 6,192 & 2,749 \\
\hline \multirow[t]{2}{*}{ Upper extremity } & $\mathrm{N}$ & 18 & 5 & 14 & 1 & 18 & 1 & 1 & - & 4 & 1 & 9 & 10 \\
\hline & $\mathrm{n}$ & 25,708 & 1,783 & 22,427 & 1,498 & 25,251 & 401 & 56 & - & 2,602 & 529 & 4,647 & 21,882 \\
\hline \multirow[t]{2}{*}{ All areas } & $\mathrm{N}$ & 8 & 1 & 6 & 1 & 8 & - & - & - & 3 & 1 & 2 & 2 \\
\hline & $\mathrm{n}$ & 3,114 & 12 & 1,604 & 1,498 & 3,114 & - & - & - & 1,960 & 433 & 231 & 490 \\
\hline \multirow[t]{2}{*}{ Total $^{4}$} & $\mathrm{~N}$ & 49 & 7 & 37 & 5 & 45 & 2 & 2 & $\mathrm{n} / \mathrm{a}$ & $n / a$ & $\mathrm{n} / \mathrm{a}$ & $\mathrm{n} / \mathrm{a}$ & $\mathrm{n} / \mathrm{a}$ \\
\hline & $\mathrm{n}$ & 88,158 & 12,632 & 64,893 & 10,633 & 87,653 & 422 & 83 & $\mathrm{n} / \mathrm{a}$ & $n / a$ & $n / a$ & $n / a$ & $\mathrm{n} / \mathrm{a}$ \\
\hline
\end{tabular}

Excessive being associated with higher prevalence of symptoms

${ }^{2}$ Excessive being associated with lower prevalence of symptoms

${ }^{3}$ Pooled OR (with $95 \% \mathrm{Cl}$ ) of $1.31[1.101 .56]$.

${ }^{4}$ Two articles reported on data from the same study, for which the $\mathrm{n}$ in the article with the highest number of participants was used.

$\mathrm{N}=$ number of studies

$\mathrm{n}=$ number of participants 
The methodological quality of all included articles is shown in Appendix 8. The average methodological quality of the included articles was 0.79 (SD: 0.16 ) out of 1 , ranging from 0.23 to 1.00 , with 32 articles describing a study considered to have high $(\geq 0.75)$ methodological quality. Data extracted from the selected articles are presented in Appendix 9. Seven articles reported on a cross-sectional case-control study[20 2931324445 52], 38 a cross-sectional observational study[21-28 30 34-36 38-43 46-51 53-57 59-66 68] and five articles a longitudinal observational study[17 333758 67]. Forty-six articles reported occupational standing assessed by self-reports[17 20 22-31 33-46 48-55 57-68], two articles described occupational standing assessed by observations[47 56] and two other articles described objectively measured (using accelerometers) occupational standing[21 32]. A total of 46 articles reported on samples of workers recruited from a general (not exclusively worker) population and/or a work population[17 20-23 26-43 45-51 53-68], while four others reported on data from samples of workers that were recruited through a clinical setting[24 2544 52]. A total of 21 articles reported about random samples of the general (working) population or samples of mixed occupational groups[17 24-26 29323337 43-45 4748505255 58-61 66] and 29 articles reported about specific occupational groups, including health care workers[21-23 2731 34-36 39-41 4649536364 67], factory and assembly workers[28 3056 57], teachers[54 68], farmers[51 65] and construction workers[38].

\section{Low-back symptoms}

A total of 39 articles ( $n=82,229$ participants in total) reported on the association of occupational standing with back symptoms, of which 33 focused on low-back symptoms[17 21 23-27 29 33-35 38-40 44-46 4950 54-62 64-68] and six on back symptoms in general[22 
28303143 63] (all referred to as low-back symptoms from here). Sixteen articles (13 crosssectional studies[28 3839 44-46 50 59-62 64 66] and three longitudinal studies[33 58 67], $\mathrm{n}=54,392$ participants in total) provided sufficiently homogenous information to conduct a meta-analysis, pooling exposure-outcome associations on excessive occupational standing and low-back symptoms. This resulted in a pooled OR (with 95\% Cl) of 1.31[1.10 1.56], with $\mathrm{I}^{2}=90 \%$ heterogeneity (Figure 2 ; Table 2). Associations found in studies with longitudinal study designs (1.17[0.64 2.14]) were not statistically significant and the point estimate was slightly weaker compared to studies with cross-sectional study designs (1.32[1.09 1.59]).

Table 2. Summary of findings from meta-analyses describing the association of occupational standing and low-back symptoms. The upper rows show the findings of the main model (Figure 2 ) while the remaining rows show the findings of the sensitivity analyses (Appendices 10 13).

\begin{tabular}{|c|c|c|c|c|c|}
\hline Subgroup factor & Subgroups & $\begin{array}{l}\text { Odds Ratio } \\
\text { (with 95\% Cl) }\end{array}$ & \multicolumn{2}{|c|}{ Test for subgroup difference } & Reference \\
\hline \multirow[t]{2}{*}{ Study design } & Cross-sectional & 1.32 [1.09 1.59] & & & \\
\hline & Total & $1.31[1.101 .56]$ & 0.14 & 0.71 & Figure 2 \\
\hline \multirow[t]{2}{*}{ Cut-off value for excessive standing } & 2 hours & 1.34 [1.08 1.65] & & & \\
\hline & 4 hours & 1.31 [1.06 1.61] & & & \\
\hline \multirow[t]{3}{*}{ Adjustment for confounders } & Unadjusted & $1.32[1.09$ 1.59] & & & \\
\hline & Adjusted & $1.23[1.021 .47]$ & & & \\
\hline & Total & $1.29\left[\begin{array}{lll}1.13 & 1.48\end{array}\right]$ & 0.31 & 0.58 & Appendix 11 \\
\hline \multirow[t]{2}{*}{ Study population } & General study population & $1.40[1.201 .62]$ & & & \\
\hline & Specific study population & $1.24[0.861 .78]$ & & & \\
\hline
\end{tabular}

$\mathrm{Cl}=$ Confidence interval

Sensitivity analyses showed that the association of excessive occupational standing and low-back symptoms did not statistically differ when the threshold for excessive occupational standing was set at 2 hours/workday compared to 4 hours/workday $\left(\chi^{2}=0.02\right.$, $p=0.88 ;$ Table 2, Appendix 10). Exposure-outcome associations unadjusted for confounders showed apparently stronger associations (1.32[1.09 1.59]) than those adjusted for confounders (1.23[1.02 1.47]), (Table 2, Appendix 11). Exposure-outcome associations from 
studies on samples of workers in general appeared stronger (1.40[1.20 1.62]) than from those on samples of specific occupational groups (1.24[0.86 1.78]), (Table 2, Appendix 12). Exposure-outcome association from studies with high methodological quality showed apparently stronger associations $(1.38[1.161 .64])$ than those from studies with low methodological quality (1.25[0.85 1.82]), (Table 2, Appendix 13). Regarding publication bias, visual inspection of the funnel plot (Appendix 14) suggested some degree of asymmetry with some larger studies reporting lower ORs than smaller studies.

The remaining 23 studies ( $n=27,899$ participants) that reported on the association between occupational standing and low-back symptoms could not be used in our metaanalysis as insufficient homogeneous evidence was provided. Nine studies reported on significant positive (i.e., excessive standing being associated with the occurrence of low-back symptoms) associations[17 21243035404963 68], eight studies reported on comparable but non-significant associations[22 2527293455 56 65] while in another six studies associations of occupational standing and low-back symptoms were assessed, but the outcomes of these associations were not reported (nor provided by the authors upon request)[23 26 54] [31 43 57]. These studies varied substantially in their definitions for exposure, outcome and exposure-outcome associations. Regarding variation in definitions of exposure for example, one study showed that there was a significantly higher prevalence of low-back symptoms for workers who reported that their 'work was hampered by standing' compared to workers who reported that their 'work was hampered by sitting' (OR with 95\% $\mathrm{Cl} ; 3.07[1.885 .01][31]$. In comparison, Hill and colleagues showed that the prevalence of low-back symptoms was higher by every hour of occupational standing, though confidence intervals were wide (OR with 95\% Cl; 2.96[0.73 12.10])[34]. Substantial heterogeneity existed in the definitions of low-back symptoms (e.g., with studies reporting on pain, 
discomfort and complaints, acute and chronic and even low-back symptoms exacerbated by occupational standing)[24 25]. Finally, some studies reported on exposure-outcome associations using metrics other than ORs, such as correlations[40], prevalence rates (PRs)[29 56] and hazard ratios (HR)[17].

\section{Lower extremity symptoms}

A total of 14 articles ( $n=31,924$ participants) reported on the association between occupational standing and lower extremity symptoms. Three articles were on lower extremity symptoms in general[29 31 43], one on hip/knee/feet symptoms combined[17], two on hip symptoms[52 65], six on knee symptoms[37 46485057 65], two on feet symptoms[47 48], two on upper leg symptoms[28 48] and three on lower leg symptoms[28 $3548]$.

Eight studies reported that excessive occupational standing was significantly associated with a higher prevalence of lower extremity symptoms (with point estimate ORs ranging from 1.23 to 3.95)[17 28313543 46-48]. Four studies reported comparable but non-statistically significant exposure-outcome associations (with point estimate ORs ranging from 1.10 to 1.70)[29 3752 65]. One study found that excessive occupational standing was associated with a significantly lower prevalence of lower extremity symptoms [65]. There were an additional four studies in which the association of occupational standing and lower extremity symptoms was assessed, but the outcomes of these associations were not reported[47 50 57] [17].

\section{Upper extremity symptoms}


A total of 18 articles ( $n=25,708$ participants) reported on the association of occupational standing and upper extremity symptoms, of which three articles focused on upper extremity symptoms in general[29 31 43], five on neck/shoulder symptoms[17 273256 68], 10 on neck symptoms[22 2834354043465763 65], seven on shoulder symptoms[22 28343550 57 63], seven on forearm/hand and/or finger symptoms[17 2228345056 65] and one on arm-only symptoms[28].

Four studies reported excessive occupational standing to be significantly associated with a higher prevalence of upper extremity symptoms[17 3132 40], nine studies reported comparable but non statistically significant exposure-outcome associations[22 28293446 565763 68] while one study found that excessive occupational standing was associated with a lower prevalence of upper extremity symptoms[28]. There were another ten studies in which the association of occupational standing and upper extremity symptoms was assessed, but the outcomes of these associations were not reported[17 27283135434650 $5765]$.

\section{Symptoms in any body area}

Eight articles ( $n=3,114$ participants) reported on the association of occupational standing with musculoskeletal symptoms in any body area[17 203641424651 53]. Three studies reported that excessive occupational standing was significantly associated with a higher prevalence of symptoms. For example, significant associations for excessive standing with symptoms were found for standing more compared to less than 30 minutes/hour (HR with $95 \% \mathrm{Cl}) 1.6[1.22 .3])[17]$ and for standing more or less than 4 hours/workday (OR with 95\% Cl) 3.67[1.88 7.17])[51]. One study reported excessive occupational standing to be associated with a reduced prevalence of symptoms compared to rarely standing (OR; 
$0.56[0.340 .94])[36]$. Two studies reported on non-significant associations of occupational standing and symptoms in any body area[41 53]. Two studies in which the association of occupational standing and lower extremity symptoms was assessed reported no outcomes of these associations [46] [42].

\section{DISCUSSION}

Meta-analysis evidence from 16 articles with 54,392 participants suggested a statistically significant association between excessive occupational standing and the occurrence of lowback symptoms with a pooled OR (with $95 \% \mathrm{Cl}$ ) of 1.31[1.10 1.56]. The association remained whether the cut-off value for excessive occupational standing was 2 or 4 hours/workday, however due to insufficient data we could not explore other cut-off values (e.g., 6 hours/workday). Therefore, at present we cannot draw conclusions on the dose-response association of excessive standing and low-back symptoms (i.e., how much standing should be considered excessive). Also evidence from studies not analysed in the meta-analysis (23 articles with $n=27,899$ participants) indicated an association of excessive occupational standing with the occurrence of low-back symptoms. Our findings are broadly in line with what has been reported in a (non-systematic) review previously[12]. Further evidence (from high quality, longitudinal studies using objectively measured occupational standing) and data from laboratory studies is needed to help determine the exposure-outcome relationship, understand the mechanisms (e.g., muscle fatigue[69] and postural changes[70 71]), and provide evidence for thresholds of excessive standing.

Although we were not able to perform a meta-analysis for the association of occupational standing and lower limb symptoms, the available evidence suggests (although inconclusively) an association between excessive occupational standing and the occurrence 
of lower extremity symptoms. These findings are in line with what has been reported before[16 66]. Similar to the associations for occupational standing with low-back symptoms, future epidemiological and laboratory research may help to explain the association between excessive occupational standing and lower extremity symptoms, may provide evidence for thresholds of excessive standing and should be explored to understand the mechanisms (e.g. muscle fatigue[72] and other non-musculoskeletal vascular mechanisms such as swelling[73] due to blood pooling in the lower limbs[69]). Evidence to date does not indicate a significant association of occupational standing and upper limb symptoms (either positive or negative).

\section{Methodological considerations}

Substantial evidence on the association of occupational standing with musculoskeletal symptoms was found (with data from $n=88,158$ participants). However due to the large heterogeneity between studies, data were difficult to synthesise. Sources of heterogeneity included: differences in the definitions of the exposure (i.e., occupational standing) and outcome (i.e., musculoskeletal symptoms); differences in study designs and study samples; and, the methodological quality of the identified studies.

The majority of the articles described studies that were based on cross-sectional designs, and thus inferences in regards to causality - including the direction of the associations - cannot be determined. While the occurrence of musculoskeletal symptoms as a result of exposure to occupational standing is discussed in most of the identified evidence (taking a traditional ergonomics perspective), it could also be possible that participants with symptoms adopt different activity behaviours than participants without symptoms [74], with variation in posture often seen as a strategy for relief from pain[75]. In the subset of studies 
that used a longitudinal design a weaker and not statistically significant association was found, reinforcing the need for caution in interpreting the cross-sectional findings.

The vast majority of the studies assessed occupational standing by self-report measures. Objective measures of occupational exposure are preferred[76], and are better able to detect true exposure-outcome associations[77]. Moreover, objective measures (e.g. using posture based monitors) allow for a more detailed assessment of activities (such as standing) that include not just the total amount of activities, but also patterns of activities. For example, a single four-hour period of static standing is likely to have different musculoskeletal consequences than four hours of standing accrued in short (e.g. $<20$ minute) bouts throughout the workday. There was also substantial variation in the operationalisation of occupational standing (e.g., standing in hours/day, hours/week, minutes/hour or even years of exposure). Similarly, the variation in the definitions of musculoskeletal symptoms were substantial, with variation in period (e.g., symptoms in the last 7 days, last month or last year), operationalisation of the symptoms (e.g., defining the intensity of symptoms or the yes/no presence of symptoms), and body area of symptoms. Harmonizing certain definitions would enhance synthesis of the evidence.

We found stronger associations in studies with high methodological quality compared to those with low methodological quality, supporting the value of high quality studies. It should be noted though that the methodological quality scale did not distinguish cross-sectional from longitudinal studies. One of the issues around methodological quality was the adjustment for confounders (or not). Heterogeneity in adjustments for confounding (i.e., in the variables -if any- used) was too great to allow meta-analysis on associations adjusted for confounders. However, sensitivity analysis showed weaker associations among evidence from studies that adjusted for confounders. This suggests the importance of 
considering confounding or mediating variables (including gender, age, other physical or mental work demands and previous musculoskeletal symptoms) that could explain or modify the association of occupational standing with musculoskeletal symptoms. These findings suggest that the current results (that are mainly based on unadjusted associations) should be interpreted with care, and that influencing variables should be considered in future research.

Studies with samples that included various occupations (i.e., a combination of various occupations or random samples of a general working population) had stronger exposureoutcome associations than those that were reported from specific occupational groups (e.g., health care, factory or construction workers), although no significant subgroup differences were observed. These specific occupational groups are likely to have a similar occupational exposure (i.e., either standing a lot, or sitting a lot) and a lack of within-group variation in occupational standing and symptoms may explain the weaker exposure-outcome associations found[78]. The 'healthy-worker-effect', in which workers without symptoms are more likely to remain in physically demanding jobs[79], may also play a role in these findings.

With some level of asymmetry in the funnel plot from studies that were included in the meta-analysis and a number of studies not reporting on (potentially non-significant) associations, publication bias might be present. Current results should therefore be interpreted with caution.

\section{CONCLUSION}

This systematic review found evidence for associations between 'excessive' occupational standing and the occurrence of low-back symptoms (pooled OR of 1.31[1.10 1.56]) and 
(inconclusively) lower extremity symptoms. The evidence did not support a significant or meaningful association of occupational standing with upper extremity symptoms. More information from high quality, longitudinal studies using objectively measured occupational standing and well-characterised symptom outcomes is needed in order to provide more definitive evidence to inform good work design.

\section{COMPETING INTEREST}

There were no conflicts of interest reported by the authors.

\section{ACKNOWLEDGEMENTS}

GNH was supported by a NHMRC Career Development Fellowship (NHMRC \#108029). DD was supported by a NHMRC Senior Research Fellowship (NHMRC \#1078360). LS was supported by a NHMRC Senior Research Fellowship (NHMRC \#1019980). CGM was supported by a NHMRC Principal Research Fellowship (NHMRC \#1103022). We are grateful for the financial assistance from the Victorian Government's OIS Program.

\section{CONTRIBUTORSHIP}

PC, LW, SP and JWS conducted literature screening and data extraction of all included papers. LR and DMB conducted the literature search in electronic data bases. All authors (PC, LW, SP, JWS, LR, DMB, CM, GNH, DWD and LMS) analysed the data and reviewed the manuscript for important intellectual content. LMS is guarantor. 
Figure 1. Flow chart, depicting the procedure of selecting articles.

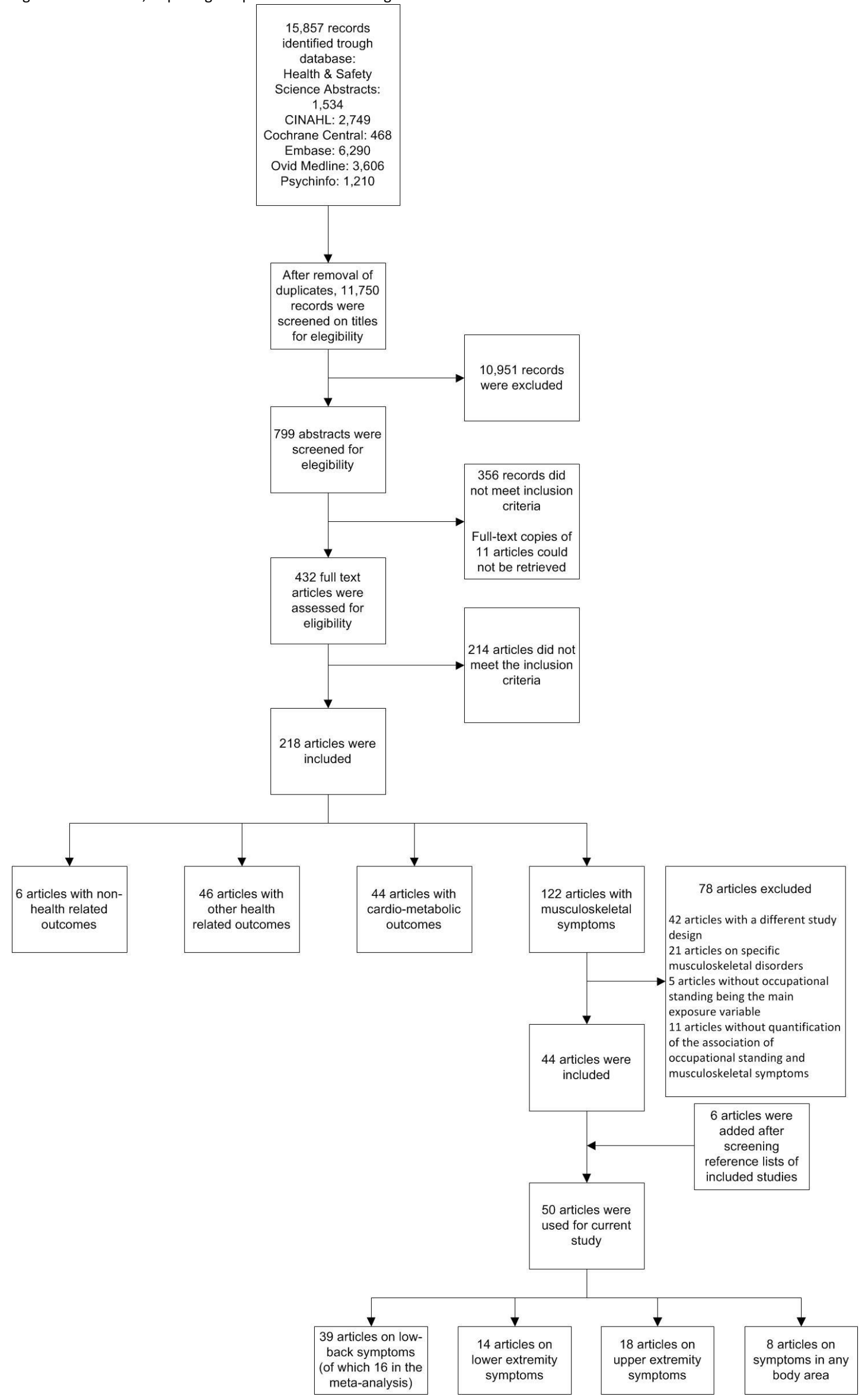


Figure 2. Forest plot of the unadjusted association of occupational standing (not-excessive standing versus excessive standing, adopting a 4 hours/workday cut-off value when possible) with low-back symptoms. Individual study, as well as pooled exposure-outcome, associations are presented. Data from cross-sectional (upper panel) as well as longitudinal (lower panel) study designs are shown. SE = standard error; $\mathrm{Cl}=$ confidence interval; IV = inverse variance.

Odds Ratio

1.1.1 Cross-sectiona

Chandraskan 2003

Kaneda 2001

Levangie 1999

Li 2012

Mehrdad 2012

Nahit 2001

Svensson 1983

Svensson 1989

Tissot 2009

Tomita 2010

Vahdati 2014

Xu 1997

Subtotal $(95 \% \mathrm{Cl})$

log[Odds Ratio]
Karahan 2009

SE Weight IV, Random, $95 \% \mathrm{Cl}$

$\begin{array}{llll}0.095 & 0.194 & 6.3 \% & 1.10[0.75,1.61]\end{array}$

$\begin{array}{llll}-0.186 & 0.052 & 8.8 \% & 0.83[0.75,0.92]\end{array}$

$\begin{array}{llll}0.557 & 0.13 & 7.6 \% & 1.75[1.35,2.25]\end{array}$

$\begin{array}{llll}0.013 & 0.255 & 5.2 \% & 1.01[0.61,1.67]\end{array}$

$\begin{array}{llll}0.488 & 0.051 & 8.8 \% & 1.63[1.47,1.80]\end{array}$

$\begin{array}{llll}0.538 & 0.288 & 4.7 \% & 1.71[0.97,3.01]\end{array}$

$\begin{array}{llll}0.279 & 0.178 & 6.7 \% & 1.32[0.93,1.87]\end{array}$

$\begin{array}{llll}0.429 & 0.176 & 6.7 \% & 1.54[1.09,2.17]\end{array}$

$\begin{array}{llll}0.346 & 0.135 & 7.5 \% & 1.41[1.08,1.84]\end{array}$

$\begin{array}{llll}0.273 & 0.054 & 8.7 \% & 1.31[1.18,1.46]\end{array}$

$\begin{array}{llll}1.359 & 0.766 & 1.2 \% & 3.89 \\ 0.87,17.47]\end{array}$

$\begin{array}{llll}-2.885 & 1.062 & 0.7 \% & 0.06[0.01,0.45]\end{array}$

$\begin{array}{llll}0.361 & 0.065 & 8.6 \% & 1.43[1.26,1.63]\end{array}$

$81.3 \% \quad 1.32[1.09,1.59]$

Heterogeneity: $\mathrm{Tau}^{2}=0.08 ; \mathrm{Chi}^{2}=115.56, \mathrm{df}=12(\mathrm{P}<0.00001) ; \mathrm{I}^{2}=90 \%$

Test for overall effect: $Z=2.90(P=0.004)$

1.1.2 Longitudinal

Harkness 2003

Sterud 2013

Yip 2004

Subtotal $(95 \% \mathrm{Cl})$

$\begin{array}{rrr}0.09 & 0.179 & 6.6 \% \\ 0.664 & 0.079 & 8.4 \%\end{array}$

$\begin{array}{rrr}0.528 & 0.367 \quad 3.6 \%\end{array}$

$18.7 \%$

$1.09[0.77,1.55]$

$1.94[1.66,2.27]$

$0.59[0.29,1.21]$

$1.17[0.64,2.14]$

Heterogeneity: Tau $^{2}=0.24 ; \mathrm{Chi}^{2}=17.23, \mathrm{df}=2(\mathrm{P}=0.0002) ; \mathrm{I}^{2}=88 \%$

Test for overall effect: $Z=0.51(P=0.61)$

Total $(95 \% \mathrm{Cl})$

$100.0 \% \quad 1.31[1.10,1.56]$

Heterogeneity: Tau $^{2}=0.09 ; \mathrm{Chi}^{2}=147.25, \mathrm{df}=15(\mathrm{P}<0.00001) ; \mathrm{I}^{2}=90 \%$

Test for overall effect: $Z=3.00(P=0.003)$

Test for subqroup differences: $\mathrm{Chi}^{2}=0.14, \mathrm{df}=1(\mathrm{P}=0.71), \mathrm{I}^{2}=0 \%$
Odds Ratio
IV, Random, $95 \% \mathrm{Cl}$

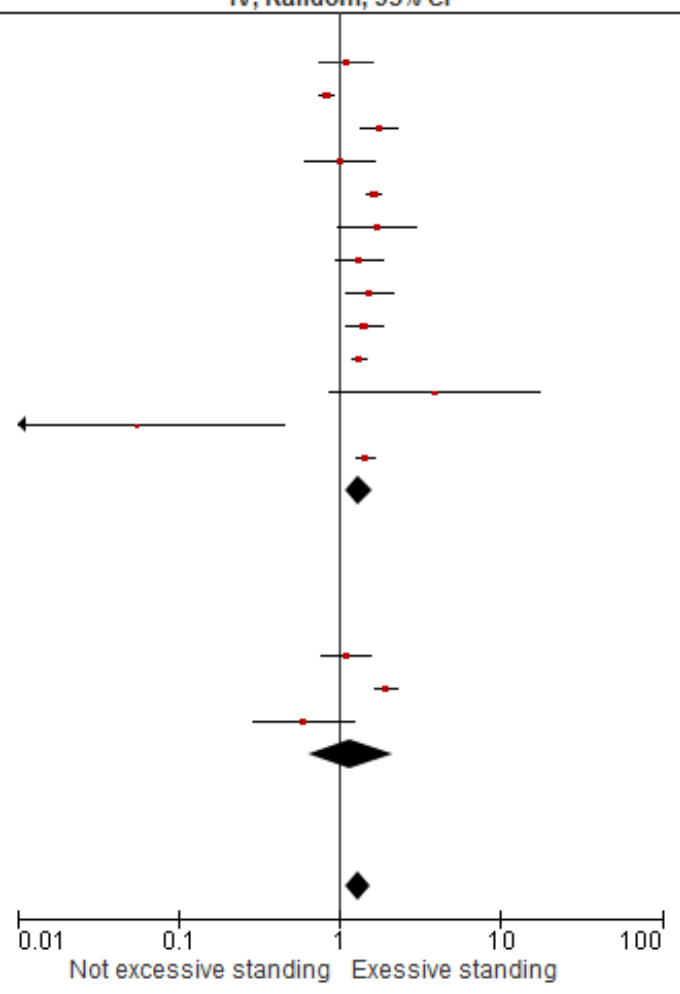


Appendix 1. Search strategy in Health \& Safety Science Abstracts (ProQuest)

\begin{tabular}{|c|c|c|}
\hline \# & Search & Results \\
\hline 1 & $\begin{array}{l}\text { (((TI,AB,SU(Standing) AND TI,AB,SU(work* OR job* OR occupation* OR employee* OR staff* OR personnel OR ergonomic*)) } \\
\text { OR (TI,AB,SU(posture* OR postural) AND TI,AB,SU(work* OR job* OR occupation* OR employee* OR staff* OR personnel } \\
\text { OR ergonomic*)) OR (TI,AB,SU(stood OR stand) NEAR/3 TI,AB,SU(work* OR job* OR occupation* OR employee* OR staff* } \\
\text { OR personnel OR ergonomic*)) OR (TI,AB,SU(stood OR stand OR standing) NEAR/4 TI,AB,SU(prolonged)) OR } \\
\text { (TI,AB,SU(upright OR posture* OR stance) NEAR/3 TI,AB,SU(prolonged)) OR (TI,AB,SU(standing OR stand OR posture* OR } \\
\text { stance) NEAR/2 TI,AB,SU(continuous)) OR (TI,AB,SU(stood OR stand OR standing) NEAR/4 TI,AB,SU(period*1)) OR } \\
\text { (TI,AB,SU(stood OR stand OR standing) NEAR/2 TI,AB,SU(time*1 OR duration)) OR (TI,AB,SU(stood OR stand OR standing) } \\
\text { NEAR/4 TI,AB,SU(hour*1)) OR (TI,AB,SU(stood OR stand OR standing) NEAR/4 TI,AB,SU(day))) }\end{array}$ & \\
\hline 2 & TI,AB,SU(trial OR trials OR study OR studies)) & \\
\hline 3 & 1 and 2 & \\
\hline 4 & $\begin{array}{l}\text { (((TI,AB,SU(Standing) AND TI,AB,SU(work* OR job* OR occupation* OR employee* OR staff* OR personnel OR ergonomic*)) } \\
\text { OR (TI,AB,SU(posture* OR postural) AND TI,AB,SU(work* OR job* OR occupation* OR employee* OR staff* OR personnel } \\
\text { OR ergonomic*)) OR (TI,AB,SU(stood OR stand) NEAR/3 TI,AB,SU(work* OR job* OR occupation* OR employee* OR staff* } \\
\text { OR personnel OR ergonomic*)) OR (TI,AB,SU(stood OR stand OR standing) NEAR/4 TI,AB,SU(prolonged)) OR } \\
\text { (TI,AB,SU(upright OR posture* OR stance) NEAR/3 TI,AB,SU(prolonged)) OR (TI,AB,SU(standing OR stand OR posture* OR } \\
\text { stance) NEAR/2 TI,AB,SU(continuous)) OR (TI,AB,SU(stood OR stand OR standing) NEAR/4 TI,AB,SU(period*1)) OR } \\
\text { (TI,AB,SU(stood OR stand OR standing) NEAR/2 TI,AB,SU(time*1 OR duration)) OR (TI,AB,SU(stood OR stand OR standing) } \\
\text { NEAR/4 TI,AB,SU(hour*1)) OR (TI,AB,SU(stood OR stand OR standing) NEAR/4 TI,AB,SU(day))) }\end{array}$ & \\
\hline 5 & (TI,AB(random* OR quasirandom* OR placebo) OR TI,AB(single-blind OR double-blind OR triple-blind OR treble-blind))) & \\
\hline 6 & 4 and 5 & \\
\hline 7 & 3 or 6 & 468 \\
\hline
\end{tabular}


Appendix 2. Search strategy in CINAHL Plus (EBSCO)

\begin{tabular}{|c|c|c|}
\hline \# & Search & Results \\
\hline 34 & $\begin{array}{l}\text { S28 AND S32 } \\
\text { LIMIT: English Language }\end{array}$ & 2,746 \\
\hline 33 & S28 AND S32 & 2,970 \\
\hline 32 & S29 OR S30 OR S31 & $1,142,504$ \\
\hline 31 & ( TI (study or studies) ) OR ( AB (study or studies) ) & 786,316 \\
\hline 30 & $\begin{array}{l}\text { (MH "Prospective Studies+") OR (MH "Case Control Studies+") OR (MH "Correlational Studies") OR (MH "Cross Sectional } \\
\text { Studies") OR (MH "Double-Blind Studies") OR (MH "Panel Studies+") OR (MH "Single-Blind Studies") OR (MH "Triple- } \\
\text { Blind Studies") OR (MH "Quasi-Experimental Studies+") OR (MH "Multicenter Studies") OR (MH "Qualitative Studies+") } \\
\text { OR (MH "Multimethod Studies") OR (MH "Field Studies") }\end{array}$ & 519,587 \\
\hline 29 & $\begin{array}{l}\text { (MH "Clinical Trials+") OR (MH "Quantitative Studies") OR PT Clinical Trial OR TI (clinical trial*) OR AB (clinical trial*) OR } \\
\text { TI random* or AB random* }\end{array}$ & 281,256 \\
\hline 28 & S8 OR S10 OR S18 OR S27 & 6,128 \\
\hline 27 & S19 OR S20 OR S21 OR S22 OR S23 OR S24 OR S25 OR S26 & 406 \\
\hline 26 & ((stood or stand or standing) N3 (period or periods) & 68 \\
\hline 25 & (standing N2 (day or time or duration)) & 183 \\
\hline 24 & ((stood or stand or standing) N4 (hour or hours)) & 42 \\
\hline 23 & ((longterm or long-term or sustained) N0 standing) & 2 \\
\hline 22 & (prolonged N2 (upright or posture)) & 29 \\
\hline 21 & (prolonged NO (orthosta* or stance)) & 7 \\
\hline 20 & (continuous* N1 (stand or standing or posture*)) & 14 \\
\hline 19 & (prolonged N4 (stand or standing)) & 99 \\
\hline 18 & S11 OR S12 OR S13 OR S14 OR S15 OR S16 OR S17 & 771 \\
\hline 17 & ((occupation* or profession* or job* or employee* or staff* or personnel) N3 (posture* or postural*)) & 83 \\
\hline 16 & ((profession or professions) N2 standing) & 7 \\
\hline 15 & (work* N1 stand) & 36 \\
\hline 14 & (work* N3 stood) & 2 \\
\hline 13 & (work* N7 posture*) & 453 \\
\hline 12 & ((work* or job* or occupation*) N2 upright) & 3 \\
\hline 11 & ((work* or job* or occupation*) N6 standing) & 244 \\
\hline 10 & S7 AND S9 & 1,888 \\
\hline 9 & TI standing or AB standing & 8,598 \\
\hline 8 & S1 AND S7 & 3,976 \\
\hline 7 & S2 OR S3 OR S4 OR S5 OR S6 & 981,422 \\
\hline 6 & (MH "Ergonomics") OR (MH "Task Performance and Analysis") & 13,274 \\
\hline 5 & $\begin{array}{l}\text { (MH "Absenteeism") OR (MH "Sick Leave") OR (MH "Retirement") OR (MH "Job Satisfaction") OR (MH "Job } \\
\text { Performance") }\end{array}$ & 28,005 \\
\hline 4 & $\begin{array}{l}\text { (MH "Work") OR (MH "Work Environment") OR (MH "Work Capacity Evaluation") OR (MH "Work Experiences") OR (MH } \\
\text { "Workload Measurement") OR (MH "Workload") OR (MH "Shiftwork") OR (MH "Women, Working+") OR (MH } \\
\text { "Workforce") OR (MH "Shift Workers") }\end{array}$ & 46,833 \\
\hline 3 & $\begin{array}{l}\text { (MH "Occupations and Professions") OR (MH "Health Occupations+") OR (MH "Named Groups by Occupation+") OR } \\
\text { (MH "Employment") OR (MH "Employment of Women") OR (MH "Employment of Older Workers") OR (MH } \\
\text { "Employment Status") OR (MH "Part Time Employment") }\end{array}$ & 914,979 \\
\hline 2 & $\begin{array}{l}\text { (MH "Occupational Diseases") OR (MH "Occupational-Related Injuries") OR (MH "Occupational Exposure") OR (MH } \\
\text { "Accidents, Occupational") OR (MH "Occupational Hazards") OR (MH "Occupational Health") OR (MH "Occupational } \\
\text { Health Services") OR (MH "Occupational Medicine") OR (MH "Occupational Safety") OR (MH "Occupational Science") }\end{array}$ & 53,568 \\
\hline 1 & (MH "Standing+") OR (MH "Posture") OR (MH "Balance, Postural") & 18,531 \\
\hline
\end{tabular}


Appendix 3. Search strategy in EBM Reviews - Cochrane Central Register of Controlled Trials

\begin{tabular}{|c|c|c|}
\hline \# & Search & Results \\
\hline 1 & postural balance/ or posture/ & 3,766 \\
\hline 2 & $\begin{array}{l}\text { occupational diseases/ or occupational exposure/ or occupational health/ or occupational medicine/ or Occupational } \\
\text { Injuries/ or Occupational Health Services/ or Accidents, Occupational/ }\end{array}$ & 1,473 \\
\hline 3 & Health Occupations/ or Occupations/ or exp occupational groups/ or exp Employment/ & 6,697 \\
\hline 4 & $\begin{array}{l}\text { work/ or work schedule tolerance/ or workload/ or workplace/ or Women, Working/ or Work Capacity Evaluation/ or } \\
\text { Work Simplification/ }\end{array}$ & 1,062 \\
\hline 5 & Absenteeism/ or Sick Leave/ or Retirement/ or Job Satisfaction/ & 818 \\
\hline 6 & Human Engineering/ or ergonomic*.tw. & 513 \\
\hline 7 & 2 or 3 or 4 or 5 or 6 & 8,967 \\
\hline 8 & 1 and 7 & 150 \\
\hline 9 & standing.ti. or standing.ab. /freq=2 & 1,286 \\
\hline 10 & 7 and 9 & 36 \\
\hline 11 & $\begin{array}{l}\text { ((standing adj4 (posture* or position)) and (work* or job* or occupation* or employee* or staff* or ergonomic* or } \\
\text { personnel)).mp. }\end{array}$ & 50 \\
\hline 12 & ((work* or job* or occupation*) adj7 standing).tw. & 58 \\
\hline 13 & ((work* or job* or occupation*) adj3 upright).tw. & 8 \\
\hline 14 & (work* adj8 posture*).tw. & 90 \\
\hline 15 & (work* adj4 stood).tw. & 0 \\
\hline 16 & (work* adj2 stand).tw. & 13 \\
\hline 17 & (profession*1 adj3 standing).tw. & 7 \\
\hline 18 & ((occupation* or profession* or job* or employee* or staff* or personnel) adj4 (posture* or postural*)).tw. & 19 \\
\hline 19 & 12 or 13 or 14 or 15 or 16 or 17 or 18 & 169 \\
\hline 20 & (prolonged adj5 (stand or standing)).tw. & 28 \\
\hline 21 & (continuous* adj2 (stand or standing or posture*)).tw. & 8 \\
\hline 22 & (prolonged adj (orthosta* or stance)).tw. & 3 \\
\hline 23 & (prolonged adj3 (upright or posture)).tw. & 7 \\
\hline 24 & ((longterm or long-term or sustained) adj standing).tw. & 0 \\
\hline 25 & ((stood or stand or standing) adj5 hour*1).tw. & 85 \\
\hline 26 & (standing adj2 (day or time or duration)).tw. & 97 \\
\hline 27 & ((stood or stand or standing) adj4 period*1).tw. & 75 \\
\hline 28 & 20 or 21 or 22 or 23 or 24 or 25 or 26 or 27 & 287 \\
\hline 29 & 8 or 10 or 11 or 19 or 28 & 581 \\
\hline 30 & limit 29 to english language & 468 \\
\hline
\end{tabular}


Appendix 4, search strategy in Embase (Ovid)

\begin{tabular}{|c|c|c|}
\hline \# & Search & Results \\
\hline 1 & body posture/ or standing/ & 63,339 \\
\hline 2 & $\begin{array}{l}\text { occupational disease/ or occupational health/ or occupational exposure/ or occupational hazard/ or occupational } \\
\text { health service/ or occupational safety/ or occupational accident/ or occupational medicine/ or industrial medicine/ }\end{array}$ & 190,792 \\
\hline 3 & $\begin{array}{l}\text { occupation/ or medical profession/ or nursing as a profession/ or paramedical profession/ or exp named groups by } \\
\text { occupation/ or exp employment/ }\end{array}$ & $1,327,430$ \\
\hline 4 & $\begin{array}{l}\text { work/ or work schedule/ or working time/ or workload/ or work capacity/ or work environment/ or work experience/ } \\
\text { or workplace/ }\end{array}$ & 113,731 \\
\hline 5 & absenteeism/ or job satisfaction/ or medical leave/ or retirement/ & 48,686 \\
\hline 6 & ergonomics/ & 8,684 \\
\hline 7 & 2 or 3 or 4 or 5 or 6 & $1,534,465$ \\
\hline 8 & 1 and 7 & 7,055 \\
\hline 9 & standing.ti. or standing.ab. /freq=2 & 16,406 \\
\hline 10 & 7 and 9 & 1,458 \\
\hline 11 & $\begin{array}{l}\text { ((standing adj4 (posture* or position)) and (work* or job* or occupation* or employee* or staff* or ergonomic* or } \\
\text { personnel)).mp. }\end{array}$ & 692 \\
\hline 12 & ((work* or job* or occupation*) adj7 standing).tw. & 1,069 \\
\hline 13 & ((work* or job* or occupation*) adj3 upright).tw. & 37 \\
\hline 14 & (work* adj8 posture*).tw. & 1,752 \\
\hline 15 & (work* adj4 stood).tw. & 22 \\
\hline 16 & (work* adj2 stand).tw. & 97 \\
\hline 17 & (profession*1 adj3 standing).tw. & 28 \\
\hline 18 & ((occupation* or profession* or job* or employee* or staff* or personnel) adj4 (posture* or postural*)).tw. & 249 \\
\hline 19 & 12 or 13 or 14 or 15 or 16 or 17 or 18 & 2,942 \\
\hline 20 & (prolonged adj5 (stand or standing)).tw. & 438 \\
\hline 21 & (continuous* adj2 (stand or standing or posture*)).tw. & 92 \\
\hline 22 & (prolonged adj (orthosta* or stance)).tw. & 65 \\
\hline 23 & (prolonged adj3 (upright or posture)).tw. & 129 \\
\hline 24 & ((longterm or long-term or sustained) adj standing).tw. & 27 \\
\hline 25 & ((stood or stand or standing) adj5 hour*1).tw. & 389 \\
\hline 26 & (standing adj2 (day or time or duration)).tw. & 1,018 \\
\hline 27 & ((stood or stand or standing) adj4 period*1).tw. & 477 \\
\hline 28 & 20 or 21 or 22 or 23 or 24 or 25 or 26 or 27 & 2,431 \\
\hline 29 & 8 or 10 or 11 or 19 or 28 & 12,029 \\
\hline 30 & limit 29 to (clinical trial or randomized controlled trial or controlled clinical trial or multicenter study) & 552 \\
\hline 31 & (random* or quasirandom* or trial or trials or placebo).tw. or clinical trial*.mp. & $2,003,888$ \\
\hline 32 & cohort analysis/ or case control study/ or longitudinal study/ or prospective study/ or retrospective study/ & 896,544 \\
\hline 33 & observational study/ or quasi experimental study/ or clinical study/ or intervention study/ or prevention study/ & 199,113 \\
\hline 34 & crossover procedure/ or controlled study/ or randomization/ & $4,565,179$ \\
\hline 35 & ((single or double or triple or treble) adj (blind* or mask*)).tw. & 170,885 \\
\hline 36 & (study or studies).tw. & 805,8649 \\
\hline 37 & 31 or 32 or 33 or 34 or 35 or 36 & $1,103,7922$ \\
\hline 38 & 29 and 37 & 7,344 \\
\hline 39 & 30 or 38 & 7,344 \\
\hline 40 & exp animal/ not human.sh. & $4,480,661$ \\
\hline 41 & 39 not 40 & 6,923 \\
\hline 42 & limit 41 to english language & 6,290 \\
\hline
\end{tabular}


Appendix 5. Search strategy in Ovid MEDLINE.

\begin{tabular}{|c|c|c|}
\hline \# & Search & Results \\
\hline 1 & postural balance/ or posture/ & 66,510 \\
\hline 2 & $\begin{array}{l}\text { occupational diseases/ or occupational exposure/ or occupational health/ or occupational medicine/ or Occupational } \\
\text { Injuries/ or Occupational Health Services/ or Accidents, Occupational/ }\end{array}$ & 164,970 \\
\hline 3 & Health Occupations/ or Occupations/ or exp occupational groups/ or exp Employment/ & 500,223 \\
\hline 4 & $\begin{array}{l}\text { work/ or work schedule tolerance/ or workload/ or workplace/ or Women, Working/ or Work Capacity Evaluation/ or } \\
\text { Work Simplification/ }\end{array}$ & 50,324 \\
\hline 5 & Absenteeism/ or Sick Leave/ or Retirement/ or Job Satisfaction/ & 37,021 \\
\hline 6 & Human Engineering/ or ergonomic*.tw. & 12,042 \\
\hline 7 & 2 or 3 or 4 or 5 or 6 & 675,628 \\
\hline 8 & 1 and 7 & 3,706 \\
\hline 9 & standing.ti. or standing.ab. /freq=2 & 12,325 \\
\hline 10 & 7 and 9 & 579 \\
\hline 11 & $\begin{array}{l}\text { ((standing adj4 (posture* or position)) and (work* or job* or occupation* or employee* or staff* or ergonomic* or } \\
\text { personnel)).mp. }\end{array}$ & 435 \\
\hline 12 & ((work* or job* or occupation*) adj7 standing).tw. & 734 \\
\hline 13 & ((work* or job* or occupation*) adj3 upright).tw. & 30 \\
\hline 14 & (work* adj8 posture*).tw. & 1,239 \\
\hline 15 & (work* adj4 stood).tw. & 16 \\
\hline 16 & (work* adj2 stand).tw. & 71 \\
\hline 17 & (profession*1 adj3 standing).tw. & 17 \\
\hline 18 & ((occupation* or profession* or job* or employee* or staff* or personnel) adj4 (posture* or postural*)).tw. & 173 \\
\hline 19 & 12 or 13 or 14 or 15 or 16 or 17 or 18 & 2,058 \\
\hline 20 & (prolonged adj5 (stand or standing)).tw. & 291 \\
\hline 21 & (continuous* adj2 (stand or standing or posture*)).tw. & 67 \\
\hline 22 & (prolonged adj (orthosta* or stance)).tw. & 51 \\
\hline 23 & (prolonged adj3 (upright or posture)).tw. & 104 \\
\hline 24 & ((longterm or long-term or sustained) adj standing).tw. & 18 \\
\hline 25 & ((stood or stand or standing) adj5 hour*1).tw. & 280 \\
\hline 26 & (standing adj2 (day or time or duration)).tw. & 739 \\
\hline 27 & ((stood or stand or standing) adj4 period*1).tw. & 346 \\
\hline 28 & 20 or 21 or 22 or 23 or 24 or 25 or 26 or 27 & 1,749 \\
\hline 29 & 8 or 10 or 11 or 19 or 28 & 7,017 \\
\hline 30 & (controlled clinical trial or randomized controlled trial).pt. & 467,024 \\
\hline 31 & (random* or quasirandom* or trial or trials or placebo).tw. or clinical trial*.mp. & $1,395,354$ \\
\hline 32 & $\begin{array}{l}\text { case-control studies/ or retrospective studies/ or cohort studies/ or longitudinal studies/ or follow-up studies/ or } \\
\text { prospective studies/ or cross-sectional studies/ or epidemiologic studies/or intervention studies/ }\end{array}$ & $1,693,552$ \\
\hline 33 & control groups/ or cross-over studies/ or double-blind method/ or random allocation/ or single-blind method/ & 239,123 \\
\hline 34 & $\begin{array}{l}\text { ((case-control or cross-sectional or cohort* or (follow-up or followup or observational or longitudinal or prospective or } \\
\text { retrospective or epidemiologic* or intervention* or incidence or prevalence)) adj (study or studies)).tw. }\end{array}$ & 566,533 \\
\hline 35 & ((single or double or triple or treble) adj (blind* or mask*)).tw. & 124,949 \\
\hline 36 & case reports/ or comparative study/ or evaluation studies/ or multicenter study/ or twin study/ or validation studies/ & $3,709,309$ \\
\hline 37 & (comparative study or evaluation studies or multicenter study or observational study or validation studies).pt. & $2,021,391$ \\
\hline 38 & (study or studies).tw. & $5,922,027$ \\
\hline 39 & 30 or 31 or 32 or 33 or 34 or 35 or 36 or 37 or 38 & $9,419,258$ \\
\hline 40 & 29 and 39 & 4,408 \\
\hline 41 & exp animals/ not humans.sh. & $3,982,927$ \\
\hline 42 & 40 not 41 & 4,103 \\
\hline 43 & limit 42 to english language & 3,606 \\
\hline
\end{tabular}


Appendix 6. Search strategy in PsycINFO. (Ovid)

\begin{tabular}{|c|c|c|}
\hline$\#$ & Searches & Results \\
\hline 1 & posture/ & 4,286 \\
\hline 2 & $\begin{array}{l}\text { occupational exposure/ or occupational health/ or occupational safety/ or work related illnesses/ or industrial } \\
\text { accidents/ }\end{array}$ & 5,846 \\
\hline 3 & exp personnel/ or occupations/ or working women/ or exp employment status/ & 344,148 \\
\hline 4 & $\begin{array}{l}\text { working conditions/ or work scheduling/ or work load/ or workday shifts/ or working space/ or job characteristics/ or } \\
\text { work rest cycles/ }\end{array}$ & 26,709 \\
\hline 5 & $\begin{array}{l}\text { job satisfaction/ or job performance/ or employee productivity/ or employee characteristics/ or productivity/ or } \\
\text { employee efficiency/ or Employee Absenteeism/ }\end{array}$ & 36,744 \\
\hline 6 & Human Factors Engineering/ or ergonomic*.tw. & 7,220 \\
\hline 7 & 2 or 3 or 4 or 5 or 6 & 382,120 \\
\hline 8 & 1 and 7 & 441 \\
\hline 9 & standing.ti. or standing.ab. /freq=2 & 2,209 \\
\hline 10 & 7 and 9 & 198 \\
\hline 11 & $\begin{array}{l}\text { ((standing adj4 (posture* or position)) and (work* or job* or occupation* or employee* or staff* or ergonomic* or } \\
\text { personnel)).mp. }\end{array}$ & 89 \\
\hline 12 & ((work* or job* or occupation*) adj7 standing).tw. & 412 \\
\hline 13 & ((work* or job* or occupation*) adj3 upright).tw. & 3 \\
\hline 14 & (work* adj8 posture*).tw. & 379 \\
\hline 15 & (work* adj4 stood).tw. & 9 \\
\hline 16 & (work* adj2 stand).tw. & 53 \\
\hline 17 & (profession*1 adj3 standing).tw. & 12 \\
\hline 18 & ((occupation* or profession* or job* or employee* or staff* or personnel) adj4 (posture* or postural*)).tw. & 61 \\
\hline 19 & 12 or 13 or 14 or 15 or 16 or 17 or 18 & 885 \\
\hline 20 & (prolonged adj5 (stand or standing)).tw. & 41 \\
\hline 21 & (continuous* adj2 (stand or standing or posture*)).tw. & 12 \\
\hline 22 & (prolonged adj (orthosta* or stance)).tw. & 5 \\
\hline 23 & (prolonged adj3 (upright or posture)).tw. & 15 \\
\hline 24 & ((longterm or long -term or sustained) adj standing).tw. & 4 \\
\hline 25 & ((stood or stand or standing) adj5 hour*1).tw. & 33 \\
\hline 26 & (standing adj2 (day or time or duration)).tw. & 189 \\
\hline 27 & ((stood or stand or standing) adj4 period*1).tw. & 56 \\
\hline 28 & 20 or 21 or 22 or 23 or 24 or 25 or 26 or 27 & 334 \\
\hline 29 & 8 or 10 or 11 or 19 or 28 & 1,659 \\
\hline 30 & $\begin{array}{l}\text { clinical trials/ or cohort analysis/ or followup studies/ or longitudinal studies/or retrospective studies/ or prospective } \\
\text { studies/ or experimentation/ or interdisciplinary research/ or qualitative research/ or quantitative methods/ or causal } \\
\text { analysis/ or exp experimental methods/ or exp experimental design/ }\end{array}$ & 118,841 \\
\hline 31 & random sampling/ or experiment controls/ & 1,406 \\
\hline 32 & (random* or quasirandom* or trial or trials or placebo).tw. or clinical trial*.mp. & 240,824 \\
\hline 33 & ((single or double or triple or treble) adj (blind* or mask*)).tw. & 20,454 \\
\hline 34 & (study or studies).tw. & $1,442,328$ \\
\hline 35 & 30 or 31 or 32 or 33 or 34 & $1,608,930$ \\
\hline 36 & 29 and 35 & 923 \\
\hline 37 & $\begin{array}{l}\text { limit } 29 \text { to ("0200 clinical case study" or "0400 empirical study" or "0430 followup study" or "0450 longitudinal study" or } \\
\text { "0451 prospective study" or "0453 retrospective study" or "0600 field study" or } 1400 \text { nonclinical case study or } 1600 \\
\text { qualitative study or } 1800 \text { quantitative study or } 2200 \text { twin study) }\end{array}$ & 1,080 \\
\hline 38 & 36 or 37 & 1,245 \\
\hline 39 & limit 38 to english language & 1,210 \\
\hline
\end{tabular}


Appendix 7. Methodological quality scale

$$
\text { Criteria }
$$

1. $\quad$ Question / objective sufficiently described?

2. $\quad$ Study design evident and appropriate?

3. Method of subject/comparison group selection or source of information/input variables described and appropriate?

4. $\quad$ Subject (and comparison group, if applicable) characteristics sufficiently described?

5. Outcome and (if applicable) exposure measure(s) well defined and robust to measurement / misclassification bias? Means of assessment reported?

6. $\quad$ Sample size appropriate?

7. $\quad$ Analytic methods described/justified and appropriate?

$8 . \quad$ Some estimate of variance is reported for the main results?

$9 . \quad$ Controlled for confounding?

10. $\quad$ Results reported in sufficient detail?

11. $\quad$ Conclusions supported by the results?

Summary score

Note, N/A is not a response option for items for items $1,2,4 \mathrm{~m} 10$ and 11 . The summary score was calculated as: total sum[(number of ' $y$ es' $\times 2)+($ number of 'partial' $\times 1)] /$ total possible sum[22 - (number of ' $N / A^{\prime} \times$ 2)], with a maximum possible total score of 1. 
Appendix 8. Methodological quality scores of included studies.

\begin{tabular}{|c|c|c|c|c|c|c|c|c|c|c|c|c|c|}
\hline & First author (Year) & Item 1 & Item 2 & Item 3 & Item 4 & Item 5 & Item 6 & Item 7 & Item 8 & Item 9 & Item 10 & Item 11 & Sum score \\
\hline 1 & Abd Rahman (2010) & 2 & 2 & 1 & 0 & 0 & 0 & 0 & 0 & 0 & 0 & 0 & 0.23 \\
\hline 2 & Andersen (2007) & 2 & 2 & 2 & 1 & 2 & 2 & 2 & 2 & 2 & 2 & 0 & 0.86 \\
\hline 3 & Babiolakis (2015) & 2 & 2 & 2 & 2 & 2 & 1 & 2 & 2 & 0 & 1 & 2 & 0.82 \\
\hline 4 & Barghout (2011) & 2 & 2 & 2 & 2 & 1 & 2 & 1 & 0 & 0 & 1 & 2 & 0.68 \\
\hline 5 & Bejia (2005) & 2 & 2 & 2 & 2 & 1 & 2 & 2 & 1 & 0 & 2 & 2 & 0.82 \\
\hline 6 & Bener (2013) & 2 & 2 & 1 & 2 & 1 & 2 & 2 & 2 & 1 & 1 & 1 & 0.77 \\
\hline 7 & Bener (2004) & 2 & 2 & 1 & 2 & 1 & 2 & 2 & 2 & 1 & 2 & 1 & 0.82 \\
\hline 8 & Bergquist-Ullman (1977) & 2 & 2 & 2 & 2 & 1 & 1 & 2 & 0 & 0 & 1 & 1 & 0.64 \\
\hline 9 & Bos (2007) & 2 & 2 & 2 & 2 & 2 & 2 & 2 & 2 & 2 & 1 & 1 & 0.91 \\
\hline 10 & Chandraskan (2003) & 2 & 2 & 2 & 2 & 1 & 2 & 2 & 2 & 2 & 2 & 1 & 0.91 \\
\hline 11 & da Silva(2006) & 2 & 2 & 2 & 2 & 2 & 2 & 2 & 2 & 2 & 1 & 0 & 0.86 \\
\hline 12 & Duquette (1997) & 2 & 1 & 2 & 1 & 1 & 1 & 1 & 0 & 0 & 1 & 1 & 0.50 \\
\hline 13 & Engels (1996) & 2 & 2 & 2 & 2 & 2 & 2 & 2 & 2 & 2 & 2 & 1 & 0.95 \\
\hline 14 & Hallman (2014) & 2 & 2 & 2 & 2 & 2 & 2 & 2 & 2 & 0 & 2 & 2 & 0.91 \\
\hline 15 & Harkness (2003) & 2 & 2 & 2 & 2 & 2 & 2 & 2 & 2 & 2 & 2 & 1 & 0.95 \\
\hline 16 & Hill (2009) & 2 & 2 & 2 & 2 & 1 & 0 & 2 & 2 & 1 & 2 & 1 & 0.77 \\
\hline 17 & Hou (2006) & 2 & 2 & 2 & 2 & 1 & 2 & 2 & 2 & 2 & 2 & 2 & 0.95 \\
\hline 18 & Jellad (2013) & 2 & 2 & 1 & 2 & 1 & 2 & 1 & 0 & 0 & 1 & 2 & 0.64 \\
\hline 19 & Jones (2007) & 2 & 2 & 2 & 1 & 2 & 2 & 2 & 2 & 2 & 2 & 1 & 0.91 \\
\hline 20 & Kaneda (2001) & 1 & 2 & 2 & 2 & 0 & 2 & 1 & 2 & 0 & 2 & 1 & 0.68 \\
\hline 21 & Karahan (2009) & 2 & 2 & 2 & 2 & 2 & 2 & 2 & 2 & 2 & 1 & 2 & 0.95 \\
\hline 22 & Kulcu (2010) & 2 & 2 & 2 & 2 & 2 & 2 & 1 & 0 & 0 & 1 & 2 & 0.73 \\
\hline 23 & Lehto (1991) & 2 & 2 & 2 & 1 & 1 & 1 & 1 & 1 & 2 & 1 & 1 & 0.68 \\
\hline 24 & Leino (1999) & 2 & 2 & 2 & 1 & 1 & 1 & 0 & 0 & 0 & 2 & 0 & 0.50 \\
\hline 25 & Leroux (2005) & 2 & 2 & 2 & 2 & 2 & 2 & 2 & 2 & 2 & 1 & 2 & 0.95 \\
\hline 26 & Levangie (1999) & 2 & 2 & 2 & 1 & 1 & 1 & 2 & 2 & 1 & 2 & 0 & 0.73 \\
\hline 27 & Li (2012) & 2 & 2 & 1 & 2 & 1 & 2 & 2 & 2 & 2 & 2 & 2 & 0.91 \\
\hline 28 & Mehrdad (2012) & 2 & 2 & 2 & 2 & 1 & 1 & 2 & 2 & 2 & 1 & 2 & 0.86 \\
\hline 29 & Messing (2001) & 2 & 2 & 0 & 2 & 2 & 1 & 0 & 0 & 0 & 2 & 1 & 0.55 \\
\hline 30 & Messing (2008) & 2 & 2 & 2 & 2 & 2 & 2 & 2 & 1 & 2 & 2 & 2 & 0.95 \\
\hline 31 & Mohseni-Banpei (2011) & 2 & 2 & 2 & 1 & 1 & 1 & 1 & 1 & 1 & 1 & 2 & 0.68 \\
\hline 32 & Nahit (2001) & 2 & 2 & 2 & 2 & 2 & 2 & 1 & 2 & 2 & 2 & 1 & 0.91 \\
\hline 33 & Phajan (2014) & 2 & 2 & 2 & 2 & 1 & 2 & 2 & 2 & 2 & 2 & 0 & 0.86 \\
\hline 34 & Pope (2003) & 2 & 2 & 2 & 2 & 2 & 2 & 2 & 2 & 2 & 2 & 0 & 0.91 \\
\hline 35 & Rodigari (2012) & 2 & 2 & 1 & 2 & 1 & 1 & 2 & 2 & 2 & 1 & 2 & 0.82 \\
\hline 36 & Samad (2010) & 2 & 2 & 2 & 2 & 1 & 1 & 1 & 2 & 0 & 1 & 1 & 0.68 \\
\hline 37 & Sanya (2005) & 2 & 2 & 1 & 2 & 0 & 2 & 1 & 1 & 0 & 1 & 1 & 0.59 \\
\hline 38 & Schierhout (1995) & 2 & 2 & 2 & 2 & 1 & 2 & 2 & 2 & 2 & 2 & 0 & 0.86 \\
\hline 40 & Smith (2002) & 2 & 2 & 2 & 2 & 2 & 1 & 2 & 2 & 2 & 1 & 0 & 0.82 \\
\hline 41 & Sterud (2013) & 2 & 2 & 2 & 2 & 2 & 2 & 2 & 2 & 2 & 2 & 2 & 1.00 \\
\hline 42 & Svensson (1989) & 2 & 2 & 2 & 2 & 1 & 0 & 1 & 1 & 0 & 1 & 2 & 0.64 \\
\hline 43 & Svensson (1983) & 2 & 2 & 2 & 2 & 1 & 1 & 1 & 1 & 0 & 1 & 1 & 0.64 \\
\hline 44 & Tissot (2009) & 2 & 2 & 2 & 2 & 2 & 2 & 1 & 1 & 0 & 1 & 2 & 0.77 \\
\hline 45 & Tomita (2010) & 2 & 2 & 2 & 2 & 1 & 1 & 2 & 2 & 1 & 2 & 1 & 0.82 \\
\hline 46 & Trinkoff (2003) & 2 & 2 & 2 & 1 & 2 & 2 & 2 & 2 & 1 & 1 & 0 & 0.77 \\
\hline 39 & Vahdati (2014) & 2 & 2 & 2 & 2 & 1 & 2 & 2 & 0 & 0 & 1 & 2 & 0.73 \\
\hline 47 & Xiao (2013) & 2 & 2 & 2 & 2 & 2 & 2 & 2 & 2 & 2 & 2 & 2 & 1.00 \\
\hline 48 & Xu (1997) & 2 & 2 & 2 & 1 & 2 & 2 & 2 & 1 & 2 & 1 & 1 & 0.82 \\
\hline 49 & Yip (2004) & 2 & 2 & 2 & 2 & 1 & 1 & 1 & 1 & 0 & 1 & 0 & 0.59 \\
\hline 50 & Yue (2012) & 2 & 2 & 2 & 2 & 2 & 2 & 2 & 2 & 2 & 2 & 1 & 0.95 \\
\hline
\end{tabular}




\begin{tabular}{|c|c|c|c|c|c|c|c|c|c|}
\hline $\begin{array}{l}\text { First author } \\
\text { (Year) }\end{array}$ & $\begin{array}{l}\text { Study } \\
\text { name }\end{array}$ & $\begin{array}{l}\text { Study design } \\
\text { (and } \\
\text { potentially } \\
\text { follow-up } \\
\text { period) }\end{array}$ & $\begin{array}{l}\text { Sample description } \\
\text { (n, \% Female, Age, } \\
\text { Country, } \\
\text { occupation, } \\
\text { relevant } \\
\text { inclusion/exclusion } \\
\text { criteria) }\end{array}$ & Confounders & Exposure & $\begin{array}{l}\text { Health Outcome (baseline } \\
\text { prevalence and if applicable } \\
\text { incidence during follow-up) }\end{array}$ & Exposure parameters & $\begin{array}{l}\text { Exposure-outcome } \\
\text { estimates }\end{array}$ & Quality \\
\hline $\begin{array}{l}\text { Abd Rahman } \\
\text { (2010) [20] }\end{array}$ & - & Case-control & $\begin{array}{l}\mathrm{n}=12 \\
\text { Female: - } \\
\text { Age: - } \\
\text { Country: Malaysia } \\
\text { Occupation: Car } \\
\text { tyre service workers }\end{array}$ & - & $\begin{array}{l}\text { Self-reported } \\
\text { prolonged } \\
\text { occupational standing } \\
\text { (yes/no) }\end{array}$ & 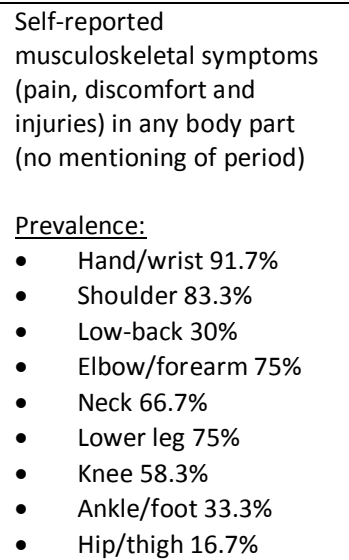 & & $\begin{array}{l}66.7 \% \text { prolonged } \\
\text { standing source of } \\
\text { injury/uncomfortable }\end{array}$ & 0.23 \\
\hline $\begin{array}{l}\text { Andersen } \\
\text { (2007) [17] }\end{array}$ & - & $\begin{array}{l}\text { Prospective (24 } \\
\text { month follow- } \\
\text { up) }\end{array}$ & $\begin{array}{l}\text { n=1498 } \\
\text { Female: - } \\
\text { Age: - } \\
\text { Country: Denmark } \\
\text { Occupation: Mixed } \\
\text { occupations (i.e., } \\
\text { administrative, } \\
\text { skilled, unskilled, } \\
\text { nurses, nurse } \\
\text { assistant, kitchen, } \\
\text { cleaning and } \\
\text { technical) } \\
\text { Exclusion: No } \\
\text { severe pain in any } \\
\text { region at baseline }\end{array}$ & $\begin{array}{ll}1 . & \begin{array}{l}\text { Partially } \\
\text { adjusted } \\
\text { model: sex, }\end{array} \\
& \text { age, } \\
\text { occupational } & \text { group } \\
\text { 2. } & \text { Fully adjusted } \\
& \text { model: for all } \\
& \text { other factors } \\
\text { (physical and } \\
\text { mental work } \\
\text { demands) }\end{array}$ & $\begin{array}{l}\text { Self-reported } \\
\text { occupational standing } \\
\text { (duration) }\end{array}$ & $\begin{array}{l}\text { Self-reported pain in the last } \\
12 \text { months in: } \\
\text { - Neck/shoulder } \\
\text { - } \quad \text { Elbow/forearm/hand } \\
\text { - } \quad \text { Hip/knee/foot } \\
\text { - } \quad \text { Any area } \\
7 \text { categories (not at all to } \\
\text { very much), with the most } \\
\text { severe categories used to } \\
\text { operationalise outcome } \\
\text { variable. } \\
\text { Prevalence: } 0 \% \\
\text { Follow-up incidence: } \\
\quad \text { Neck/shoulder: } 11.5 \% \\
\text { Elbow/forearm/hand: } \\
6.4 \% \\
\text { - Low-back: } 10.6 \% \\
\text { - Hip/knee/foot: } 9.3 \%\end{array}$ & $\begin{array}{ll}\text { - } & \begin{array}{l}\text { Not standing }>30 \\
\text { mins/hr }(n=1384)\end{array} \\
\text { - } & \begin{array}{l}\text { Standing }>30 \\
\text { mins/hr }(n=114)\end{array}\end{array}$ & $\begin{array}{l}\text { Reference } \\
\text { Neck/shoulder: } \\
\text { HR: } 1.8\left[\begin{array}{ll}1.2 & 2.9\end{array}\right]^{1} \\
\text { Elbow/forearm: } \\
\text { HR: } 2.0\left[\begin{array}{ll}1.1 & 3.7\end{array}\right]^{1} \\
\text { Low-back } \\
\text { HR: } 2.1\left[\begin{array}{ll}1.3 & 3.3\end{array}\right]^{1} \\
\text { HR: } 1.9\left[\begin{array}{ll}1.2 & 3.0\end{array}\right]^{2} \\
\text { Hip/knee/foot } \\
\text { HR: } 1.7\left[\begin{array}{ll}1.0 & 2.9\end{array}\right]^{1} \\
\text { Any region: } \\
\text { HR: } 1.7\left[\begin{array}{ll}1.1 & 2.3\end{array}\right]^{1} \\
\text { HR: } 1.6\left[\begin{array}{ll}1.2 & 2.3\end{array}\right]^{2}\end{array}$ & 0.86 \\
\hline
\end{tabular}




\begin{tabular}{|c|c|c|c|c|c|c|c|c|}
\hline & & & & & - Any region: $23.6 \%$ & & & \\
\hline $\begin{array}{l}\text { Babiolakis } \\
\text { (2015) [21] }\end{array}$ & Cross-sectional & $\begin{array}{l}\mathrm{n}=27 \\
\text { Female - } 100 \% \\
\text { Age: } 44.1 \text { (14.7) } \\
\text { years (recently } \\
\text { injured); } 44.9 \text { (14.3) } \\
\text { years (not recently } \\
\text { injured) } \\
\text { Country: Canada } \\
\text { Occupation: Nurses }\end{array}$ & - & $\begin{array}{l}\text { Objectively assessed } \\
\text { (through } \\
\text { accelerometer- } \\
\text { inclinometer) } \\
\text { occupational standing } \\
\text { (total duration and } \\
\text { bout duration) }\end{array}$ & 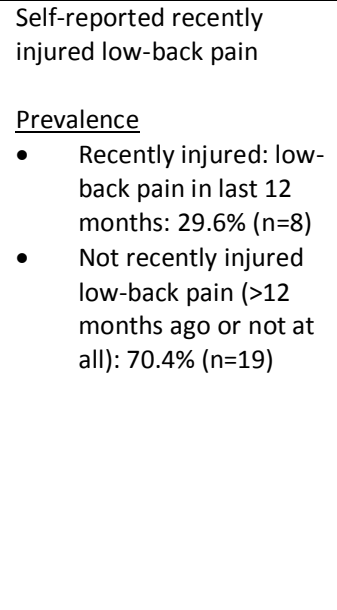 & & $\begin{array}{l}\text { Standing time } \\
\text { Recently injured: } \\
208.9(111.0) \text { minutes } \\
\text { Not recently injured: } \\
317.8(114.0) \\
\text { minutes. Significant } \\
\text { between group } \\
\text { difference } p=0.033 \\
\\
\text { Standing bout } \\
\text { Recently injured: } 19.3 \\
(18.6) \text { minutes) } \\
\text { Not recently injured: } \\
60.2(71.3) \text { minutes. } \\
\text { Significant between } \\
\text { group difference } \\
p=0.035\end{array}$ & 0.82 \\
\hline $\begin{array}{l}\text { Barghout } \\
\text { (2011) [22] }\end{array}$ & Cross-sectional & $\begin{array}{l}\mathrm{n}=200 \\
\text { Female: } 43 \% \\
\text { Age: } 40.6 \text { years } \\
\text { (males); } 40.1 \text { years } \\
\text { (females) } \\
\text { Country: Jordan } \\
\text { Occupation: } \\
\text { Dentists }\end{array}$ & & $\begin{array}{l}\text { Self-reported } \\
\text { occupational standing } \\
\text { (yes/no) }\end{array}$ & 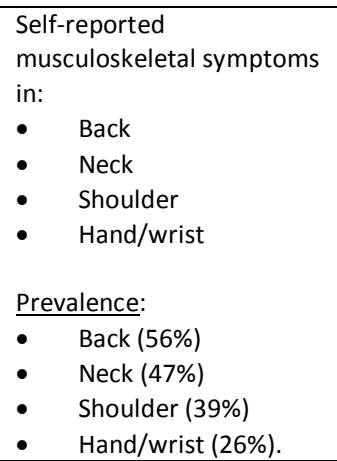 & Standing only $(n=10)$ & $\begin{array}{ll}\text { - } & \text { Hand/wrist pain } \\
& \mathrm{n}=6(60 \%) \\
\text { - } & \text { Neck pain } \mathrm{n}=5 \\
& (50 \%) \\
\text { - } & \text { Shoulder pain } \\
& \mathrm{n}=5(50 \%) \\
\text { - } & \text { Back pain } \mathrm{n}=5 \\
& (50 \%) \\
\end{array}$ & 0.68 \\
\hline $\begin{array}{l}\text { Bejia (2005) } \\
{[23]}\end{array}$ & Cross-sectional & $\begin{array}{l}\mathrm{n}=350 \\
\text { Female: } 49 \% \\
\text { Age: } 37.0(7.8) \\
\text { years } \\
\text { Country: Tunisia } \\
\text { Occupation: } \\
\text { Hospital workers }\end{array}$ & - & $\begin{array}{l}\text { Self-reported } \\
\text { occupational standing } \\
\text { (no details provided) }\end{array}$ & $\begin{array}{l}\text { Self-reported common low- } \\
\text { back pain (acute and } \\
\text { chronic low-back pain) } \\
\text { during the last } 12 \text { months. } \\
\text { Prevalence: } 50 \%\end{array}$ & Standing $\mathrm{n}=174(50 \%)$ & $\begin{array}{ll}- & \text { Low-back pain } \\
& (\mathrm{n}=176 ; 49.4 \%) \\
-\quad & \text { No low-back } \\
& \text { pain ( } \mathrm{n}=174 ; \\
& 50 \%) \\
& \\
& \end{array}$ & 0.82 \\
\hline Bener (2013) & Cross-sectional & $\mathrm{n}=1290$ & 1. Univariate & Self-reported & Self-reported low-back pain & & Low-back pain & 0.77 \\
\hline
\end{tabular}




\begin{tabular}{|c|c|c|c|c|c|c|c|c|c|}
\hline [25] & & $\begin{array}{l}\text { Female: } 41.7 \% \\
\begin{array}{l}\text { Age: } \\
-\quad<35 \text { years } \\
\quad(n=313) \\
\quad 35-44 \text { years } \\
\quad(n=483) \\
\quad 45-54 \text { years } \\
(n=805) \\
\quad \quad 255 \text { years } \\
(n=579)\end{array} \\
\text { Country: Qatar } \\
\text { Occupation: People } \\
\text { visiting } 12 \text { health } \\
\text { centres; mixed } \\
\text { occupations - not } \\
\text { working, sedentary } \\
\text { and professional, } \\
\text { clerk, business, } \\
\text { army/police }\end{array}$ & 2. & $\begin{array}{l}\text { Multivariate } \\
\text { model: } \\
\text { factors not } \\
\text { described }\end{array}$ & $\begin{array}{l}\text { prolonged standing } \\
\text { (not clear whether it is } \\
\text { just about } \\
\text { occupational standing) }\end{array}$ & $\begin{array}{l}>1 \text { day in the last } 6 \text { months. } \\
\text { Prevalence: } 59.2 \%\end{array}$ & & $\begin{array}{l}\text { exacerbated by } \\
\text { prolonged standing: } \\
\text { Males 221/595 } \\
(41.2 \%)^{1} \\
\text { Females 182/695 } \\
(29.5 \%)^{1} \\
\text { Multivariable } \\
\text { analysis' - no } \\
\text { significant } \\
\text { contribution }\end{array}$ & \\
\hline $\begin{array}{l}\text { Bener (2004) } \\
{[24]}\end{array}$ & Cross-sectional & $\begin{array}{l}\mathrm{n}=1103 \\
\text { Females: } 46.9 \% \\
\text { Age: } 34.9 \text { (13.4) } \\
\text { (males) years; } 33.5 \\
\text { (11.8) (females) } \\
\text { years } \\
\text { Country: UAE } \\
\text { Occupation: People } \\
\text { visiting health } \\
\text { centres. Mixed } \\
\text { occupations (i.e., } \\
\text { sedentary, manual } \\
\text { work, professional, } \\
\text { student, housewife) }\end{array}$ & 1. & $\begin{array}{l}\text { Multivariate } \\
\text { model: BMI, } \\
\text { weakness in } \\
\text { leg, heavy } \\
\text { lifting, regular } \\
\text { exercise, } \\
\text { smoking }\end{array}$ & $\begin{array}{l}\text { Self-reported } \\
\text { prolonged standing } \\
\text { (not clear whether it is } \\
\text { just about } \\
\text { occupational standing) }\end{array}$ & $\begin{array}{l}\text { Self-reported low-back pain } \\
\text { ( } 1 \text { day in last } 6 \text { months) } \\
\frac{\text { Prevalence: } 64.6 \%[60.7}{68.5]}\end{array}$ & $\begin{array}{l}\text { - Not prolonged } \\
\text { standing } \\
\text { - Prolonged standing }\end{array}$ & $\begin{array}{l}\text { Low-back pain } \\
\text { exacerbated by } \\
\text { prolonged standing: } \\
\text { Males } \\
\text { 136/333 (50.8\%) } \\
\text { Females } \\
\text { 272/380 (71.6\%) } \\
\text { Reference } \\
\text { RR:6.22 [4.01 9.67 }]^{1}\end{array}$ & 0.82 \\
\hline $\begin{array}{l}\text { Bergquist- } \\
\text { Ullman } \\
\text { (1977) [26] }\end{array}$ & Cross-sectional & $\begin{array}{l}\mathrm{n}=217 \\
\text { Females: } 13 \% \\
\text { Age: } 34.5 \text { (median) }\end{array}$ & - & & $\begin{array}{l}\text { Self-reported } \\
\text { occupational standing } \\
\text { - } \quad \geq 4 \mathrm{hrs} / \text { day } \\
\text { - } \quad 2-4 \mathrm{hrs} / \text { day }\end{array}$ & $\begin{array}{l}\text { Self-reported low-back pain } \\
\text { (acute or subacute pain, } \\
\text { duration }<3 \text { months with a } \\
\text { pain free year before the } \\
\text { onset of the current }\end{array}$ & $\begin{array}{ll}\text { - } & \text { Standing } \geq 4 \mathrm{hr} / \text { day } \\
\text { - } & \text { Standing 2-4 } \\
& \text { hrs/day }\end{array}$ & $\begin{array}{l}135 \text { workers } \\
39 \text { workers }\end{array}$ & 0.64 \\
\hline
\end{tabular}




\begin{tabular}{|c|c|c|c|c|c|c|c|c|c|}
\hline & & & $\begin{array}{l}\text { years } \\
\text { Country: Sweden } \\
\text { Occupation: Manual } \\
\text { and office workers } \\
\text { from automotive } \\
\text { workshop }\end{array}$ & & - $\quad<2$ hrs/day & $\begin{array}{l}\text { episode) } \\
\text { Prevalence: } 100 \%\end{array}$ & - $\quad$ Standing $<2 \mathrm{hrs} /$ day & 43 workers & \\
\hline $\begin{array}{l}\text { Bos (2007) } \\
{[27]}\end{array}$ & - & Cross-sectional & $\begin{array}{l}\mathrm{n}=3169 \\
\text { Females: } 82 \% \\
\text { Age: } 38(10) \text { years } \\
\text { Country: the } \\
\text { Netherlands } \\
\text { Occupation: Nurses } \\
\text { and x-ray } \\
\text { technicians }\end{array}$ & $\begin{array}{ll}\text { 1. Univariate } \\
\text { 2. } & \text { Multivariate } \\
\text { model: } \\
\text { physical and } \\
\text { psychosocial } \\
\text { risk factors, } \\
\text { gender, age } \\
\text { body mass, } \\
\text { height, } \\
\text { duration of } \\
\text { employment, } \\
\text { working } \\
\text { hours/ week } \\
\text { and } \\
\text { managerial } \\
\text { function. } \\
\end{array}$ & $\begin{array}{l}\text { Self-reported } \\
\text { occupational standing } \\
\text { (standing often at } \\
\text { work) }\end{array}$ & $\begin{array}{l}\text { Self-reported low-back or } \\
\text { neck/shoulder pain in last } \\
12 \text { months) } \\
\text { Prevalence: } \\
\quad \text { Low-back pain: } 75.9 \% \\
\text { - Neck/shoulder pain: } \\
59.8 \%\end{array}$ & $\begin{array}{l}\text { - } \quad \text { No standing often } \\
\text { - } \quad \text { Standing often }\end{array}$ & $\begin{array}{l}\text { Reference } \\
\text { Low-back pain: } \\
\text { Non-specialised } \\
\text { nurses }(n=1977) \\
\text { OR:1.33 [0.95 1.86] }{ }^{2} \\
\text { Operation room } \\
\text { nurses ( } n=381) \\
\text { OR:1.09 [0.49 2.38 }]^{2}\end{array}$ & 0.91 \\
\hline $\begin{array}{l}\text { Chandraskan } \\
\text { (2003) [28] }\end{array}$ & - & Cross-sectional & $\begin{array}{l}\mathrm{n}=529 \\
\text { Females: } 100 \% \\
\text { Age: } 31.2(7.4) \\
\text { years } \\
\text { Country: Malaysian } \\
\text { Occupation: Factory } \\
\text { workers }\end{array}$ & $\begin{array}{ll}\text { 1. } & \text { Univariate } \\
\text { 2. } & \text { Multivariate } \\
\text { model: work } \\
\text { factors and } \\
\text { body site }\end{array}$ & $\begin{array}{l}\text { Self-reported } \\
\text { occupational standing } \\
\text { (duration) with } \\
\text { options: } \\
\text { - } \quad \text { No standing } \\
\text { - } \quad<2 \mathrm{hrs} / \text { day } \\
\text { - } \quad 2-4 \mathrm{hrs} / \text { day } \\
\text { - } \quad \geq 4 \mathrm{hrs} / \text { day }\end{array}$ & $\begin{array}{ll}\text { Self-reported pain in the last } \\
12 \text { months (Nordic } \\
\text { questionnaire): } \\
\text { - } \quad \text { Neck } \\
\text { - } \quad \text { Shoulder } \\
\text { - } & \text { Arm } \\
\text { - } & \text { Wrist/fingers } \\
\text { - } & \text { Upper leg } \\
\text { - } & \text { Lower leg } \\
\text { - } & \text { Back } \\
\text { Prevalence: } \\
\text { - } & \text { Neck } 29.7 \% \\
\text { - } & \text { Shoulder } 44.8 \% \\
\text { - } & \text { Arm } 29.1 \% \\
\text { - } & \text { Wrist/fingers } 22.1 \% \\
\text { - } & \text { Lower leg } 48.4 \% \\
\text { - } & \text { Upper leg } 38.8 \% \\
\text { - } & \text { Back } 57.8 \%\end{array}$ & $\begin{array}{ll}\text { - } & \begin{array}{l}\text { Standing } \leq 4 \\
\text { hrs/day. }\end{array} \\
\text { - } & \begin{array}{l}\text { Standing }>4 \\
\text { hrs/day. }\end{array}\end{array}$ & $\begin{array}{l}\text { Reference } \\
\text { Neck } \\
\text { OR: } 0.8\left[\begin{array}{ll}0.5 & 1.1\end{array}\right]^{1} \\
\text { Shoulder } \\
\text { OR: } 0.9\left[\begin{array}{ll}0.6 & 1.3\end{array}\right]^{1} \\
\text { Arm } \\
\text { OR: } 1.0\left[\begin{array}{ll}0.7 & 1.4\end{array}\right]^{1} \\
\text { Wrist/fingers } \\
\text { OR: } 1.2\left[\begin{array}{ll}0.8 & 1.9\end{array}\right]^{1} \\
\text { Back } \\
\text { OR: } 1.1\left[\begin{array}{ll}0.7 & 1.5\end{array}\right]^{1} \\
\text { Lower leg } \\
\text { OR: } 4.8\left[\begin{array}{ll}3.3 & 7.1\end{array}\right]^{1} \\
\text { OR: } 3.3\left[\begin{array}{ll}2.1 & 5.3]^{2}\end{array}\right.\end{array}$ & 0.91 \\
\hline
\end{tabular}




\begin{tabular}{|c|c|c|c|c|c|c|c|c|c|c|}
\hline & & & & & & & & & $\begin{array}{l}\text { Upper leg } \\
\text { OR: } 3.1\left[\begin{array}{lll}2.1 & 4.5\end{array}\right]^{1} \\
\text { OR: } 1.8\left[\begin{array}{lll}1 & .1 & 2.9\end{array}\right]^{2}\end{array}$ & \\
\hline $\begin{array}{l}\text { da Silva } \\
\text { (2006) [29] }\end{array}$ & - & Case-control & $\begin{array}{l}\mathrm{n}=881 \\
\text { Females: } 37 \% \\
\text { Age: } 38 \text { years } \\
\text { Country: Brazil } \\
\text { Occupation: Rag- } \\
\text { pickers and } \\
\text { matched controls }\end{array}$ & 1. & $\begin{array}{l}\text { Multivariate } \\
\text { model: all } \\
\text { other physical } \\
\text { work } \\
\text { demands and } \\
\text { demographic } \\
\text { variables (i.e., } \\
\text { age gender, } \\
\text { education } \\
\text { and marital } \\
\text { status) }\end{array}$ & $\begin{array}{l}\text { Self-reported } \\
\text { occupational standing } \\
\text { (duration) on } 4 \text { point } \\
\text { scale- reduced to } 2 \\
\text { categories: } \\
\text { - Never/rarely } \\
\text { - Generally/always }\end{array}$ & $\begin{array}{l}\text { Self-reported pain in neck, } \\
\text { shoulder, elbow, } \\
\text { wrist/hands, low-back, } \\
\text { thighs, lower leg, knees, } \\
\text { ankles (Nordic) in the past } \\
12 \text { months. Pain locations } \\
\text { were combined into: } \\
\text { - Low-back } \\
\text { - } \quad \text { Lower extremity } \\
\text { - Upper extremity. } \\
\text { Prevalence: } \\
\quad \quad \text { Low-back: } 49.2 \% \text { (rag- } \\
\quad \text { pickers); } 49.1 \% \text { (non- } \\
\text { rag-pickers) } \\
\text { Upper extremity: } 35 \% \\
\text { (rag-pickers); } 36.8 \% \\
\text { (non-rag-pickers) }\end{array}$ & $\begin{array}{ll}- & \begin{array}{l}\text { Never/rarely } \\
\text { standing }\end{array} \\
\text { - } & \begin{array}{l}\text { Generally/always } \\
\text { standing }\end{array}\end{array}$ & $\begin{array}{l}\text { Reference } \\
\text { Low-back } \\
\text { PR: } 1.0\left[\begin{array}{ll}0.8 & 1.2\end{array}\right]^{1} \\
\text { Lower extremity } \\
\text { PR: } 1.1\left[\begin{array}{ll}0.9 & 1.4\end{array}\right]^{1} \\
\text { Upper extremity } \\
\text { PR: } 1.2\left[\begin{array}{ll}0.9 & 1.6\end{array}\right]^{1}\end{array}$ & 0.86 \\
\hline $\begin{array}{l}\text { Duquette } \\
\text { (1997) [30] }\end{array}$ & - & Cross-sectional & $\begin{array}{l}\mathrm{n}=176 \\
\text { Females: - } \\
\text { Age: } 36.9(10.4) \\
\text { years } \\
\text { Country: Canada } \\
\text { Occupation: } \\
\text { Assembly workers }\end{array}$ & - & & $\begin{array}{l}\text { Self-reported } \\
\text { occupational standing } \\
\text { (for long time; work } \\
\text { mainly requiring } \\
\text { standing) }\end{array}$ & $\begin{array}{l}\text { Self-reported back } \\
\text { discomfort in the previous } \\
\text { week } \\
\text { Prevalence: } 44.9 \%\end{array}$ & 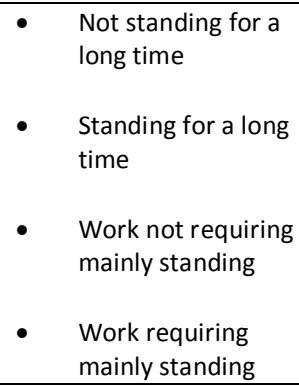 & $\begin{array}{l}\text { Reference } \\
\text { OR: } 2.9 \text { (significant) } \\
\text { Reference } \\
\text { OR: } 1.7 \text { (not } \\
\text { significant) }\end{array}$ & 0.50 \\
\hline $\begin{array}{l}\text { Engels } \\
\text { (1996) [31] }\end{array}$ & - & Case-control & $\begin{array}{l}\mathrm{n}=846 \\
\text { Females: } 89.8 \% \\
\text { Age: } 29.0(8.0) \\
\text { years } \\
\text { Country: the } \\
\text { Netherlands } \\
\text { Occupation: Nurses }\end{array}$ & $\begin{array}{l}1 . \\
2 .\end{array}$ & $\begin{array}{l}\text { Partially } \\
\text { adjusted } \\
\text { model: age, } \\
\text { sex, } \\
\text { employment } \\
\text { duration, } \\
\text { working } \\
\text { hours, having } \\
\text { managerial } \\
\text { tasks } \\
\text { Fully adjusted } \\
\text { model: as } \\
\text { above plus all }\end{array}$ & $\begin{array}{l}\text { Self-reported work } \\
\text { hampered by standing }\end{array}$ & $\begin{array}{l}\text { Self-reported complaints } \\
\text { (Validated Dutch } \\
\text { questionnaire, no } \\
\text { mentioning of period) in: } \\
\text { - Back } \\
\text { - } \quad \text { Arms and neck } \\
\text { - Legs } \\
\text { Prevalence } \\
\text { Back: } 35.9 \% \\
\text { - } \quad \text { Arm or neck: } 30.4 \% \\
\text { Leg: } 15.7 \%\end{array}$ & 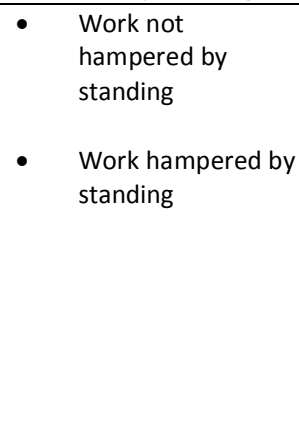 & $\begin{array}{l}\text { Reference } \\
\text { Back: } \\
\text { OR:3.07 [1.88 } 5.01]^{1} \\
\text { Arm and neck } \\
\text { OR:2.75 [1.60 } 4.72]^{1} \\
\text { Leg } \\
\text { OR:4.90 [2.84 } 8.47]^{1} \\
\text { OR:2.49 }\left[\begin{array}{ll}1.17 & 5.24\end{array}\right]^{2}\end{array}$ & 0.95 \\
\hline
\end{tabular}




\begin{tabular}{|c|c|c|c|c|c|c|c|c|c|c|}
\hline & & & & & $\begin{array}{l}\text { work related } \\
\text { factors and } \\
\text { significant } \\
\text { factors from } \\
\text { univariate } \\
\text { analysis }\end{array}$ & & & & & \\
\hline $\begin{array}{l}\text { Hallman } \\
\text { (2014) [32] }\end{array}$ & - & Case-control & $\begin{array}{l}\mathrm{n}=56 \\
\text { Females: } 45 \% \\
\text { Age: cases } 41(10) \\
\text { years; controls } 41 \\
\text { (9) years } \\
\text { Country: Sweden } \\
\text { Occupation: Office } \\
\text { and production } \\
\text { workers }\end{array}$ & - & & $\begin{array}{l}\text { Objectively measured } \\
\text { over } 7 \text { days (using } \\
\text { activPAL'TM } \\
\text { inclinometer) time } \\
\text { spent standing, } \\
\text { occupational and } \\
\text { leisure time. }\end{array}$ & $\begin{array}{l}\text { Self-reported non-traumatic } \\
\text { chronic pain (>6 months), } \\
\text { localized to the } \\
\text { neck/shoulder region (i.e., } \\
\text { primary the neck and/or } \\
\text { trapezius muscles) } \\
\text { Prevalence: } 52 \% \text { ( } 29 \text { cases, } \\
27 \text { controls) }\end{array}$ & & $\begin{array}{l}\text { Neck/shoulder pain } \\
\text { group spent more } \\
\text { time standing (16.4 } \\
\text { (8.0) } \mathrm{min} / \mathrm{hr} \text { at work } \\
\text { and } 14.3(5.1) \mathrm{min} / \mathrm{hr} \\
\text { in leisure time) than } \\
\text { controls (12.2 (8.8) } \\
\mathrm{min} / \mathrm{hr} \text { at work and } \\
11.6(5.0) \mathrm{min} / \mathrm{hr}) \\
\\
\text { Between group } \\
\text { difference: } \mathrm{p}=0.037\end{array}$ & 0.91 \\
\hline $\begin{array}{l}\text { Harkness } \\
\text { (2003) [33] }\end{array}$ & - & $\begin{array}{l}\text { Prospective } \\
\text { cohort with } 12 \\
\text { and } 24 \text { months } \\
\text { follow-up. }\end{array}$ & $\begin{array}{l}\mathrm{n}=1048 \text { (620 } \\
\text { participants without } \\
\text { pain at baseline and } \\
428 \text { participants } \\
\text { without pain after } \\
12 \text { months; } \\
\text { numbers overlap) } \\
\text { Females: } 36 \% \\
\text { Age: } 23 \text { (median) } \\
\text { years } \\
\text { Country: USA } \\
\text { Occupation: Newly } \\
\text { employed workers } \\
\text { from } 12 \text { diverse } \\
\text { occupational groups }\end{array}$ & 1. & $\begin{array}{l}\text { Partially } \\
\text { adjusted } \\
\text { model: age, } \\
\text { gender, } \\
\text { occupational } \\
\text { group; } \\
\text { Fully adjusted } \\
\text { model: as } \\
\text { above and all } \\
\text { other } \\
\text { postures }\end{array}$ & $\begin{array}{l}\text { Self-reported duration } \\
\text { of occupational } \\
\text { standing, classified } \\
\text { into: } \\
\text { - } \quad \text { No standing } \\
\text { - } \quad<15 \mathrm{mins} / \text { day } \\
\text { - } \quad 15 \mathrm{mins}-<2 \\
\text { hrs/day } \\
\text { - } \quad \geq 2 \mathrm{hrs} / \text { day }\end{array}$ & $\begin{array}{l}\text { Self-reported low-back pain } \\
\text { lasting } 24 \text { hours or longer in } \\
\text { the past month. Low-back } \\
\text { pain was assessed at } \\
\text { baseline and during the } 12 \\
\text { and } 24 \text { month follow-up. } \\
\text { Prevalence: } 0 \%\end{array}$ & 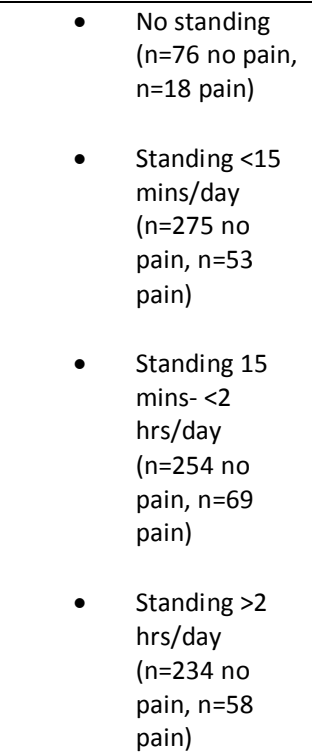 & $\begin{array}{l}\text { Reference } \\
\text { OR: } 1.1\left[\begin{array}{ll}0.6 & 2.1\end{array}\right]^{1} \\
\text { OR: } 1.0\left[\begin{array}{ll}0.5 & 1.9\end{array}\right]^{2} \\
\text { OR: } 1.6\left[\begin{array}{ll}0.8 & 2.9\end{array}\right]^{1} \\
\text { OR: } 1.4\left[\begin{array}{ll}0.7 & 2.7\end{array}\right]^{2} \\
\\
\text { OR: } 1.8\left[\begin{array}{ll}0.9 & 3.4\end{array}\right]^{1} \\
\text { OR: } 1.5\left[\begin{array}{ll}0.8 & 3.0\end{array}\right]^{2}\end{array}$ & 0.95 \\
\hline $\begin{array}{l}\text { Hill (2009) } \\
{[34]}\end{array}$ & - & Cross-sectional & $\begin{array}{l}\mathrm{n}=26 \\
\text { Females: } 100 \% \\
\text { Age: } 36.9(8.68)\end{array}$ & $\begin{array}{l}1 . \\
2 .\end{array}$ & $\begin{array}{l}\text { Univariate } \\
\text { Multivariate } \\
\text { model: } \\
\text { physical size, } \\
\text { job strain, }\end{array}$ & $\begin{array}{l}\text { Self-reported duration } \\
\text { of occupational } \\
\text { standing }\end{array}$ & $\begin{array}{l}\text { Self-reported } \\
\text { musculoskeletal symptoms } \\
\text { during last } 12 \text { months } \\
\text { (Nordic Musculoskeletal } \\
\text { Questionnaire) in: }\end{array}$ & Time spent standing & $\begin{array}{l}\text { Shoulder } \\
\text { OR:1.20 [0.49 } 2.96]^{1} \\
\text { OR:1.01 [0.27 } 3.71]^{2} \\
\text { Low-back }\end{array}$ & 0.77 \\
\hline
\end{tabular}




\begin{tabular}{|c|c|c|c|c|c|c|c|c|c|c|}
\hline & & & $\begin{array}{l}\text { years } \\
\text { Country: USA } \\
\text { Occupation: } \\
\text { Sonographers }\end{array}$ & & $\begin{array}{l}\text { time, } \\
\text { abdominal } \\
\text { girth, } \\
\text { workplace } \\
\text { variability and } \\
\text { moving } \\
\text { during study. }\end{array}$ & & $\begin{array}{ll}\text { - } & \text { Neck } \\
\text { - } & \text { Shoulders } \\
\text { - } & \text { Wrists/hands } \\
\text { - } & \text { Low-back } \\
\text { Prevalence: } \\
\text { - } & \text { Shoulder } 73 \% \\
\text { - } & \text { Low-back } 69 \% \\
\text { - } & \text { Wrist/hand 54\% } \\
\text { - } & \text { Neck } 50 \%\end{array}$ & & 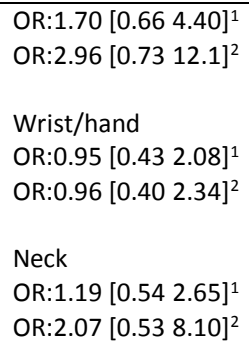 & \\
\hline $\begin{array}{l}\text { Hou (2006) } \\
{[35]}\end{array}$ & - & Cross-sectional & $\begin{array}{l}\mathrm{n}=3950 \\
\text { Females: } 100 \% \\
\text { Age: majority } \\
\text { between } 25 \text { and } 34 \\
\text { years } \\
\text { Country: Taiwan } \\
\text { Occupation: Nurses }\end{array}$ & 1. & $\begin{array}{l}\text { Multivariate } \\
\text { model: age, } \\
\text { tenure and } \\
\text { weekly work } \\
\text { hours. }\end{array}$ & $\begin{array}{l}\text { Self-reported } \\
\text { occupational standing } \\
\text { (duration) }\end{array}$ & $\begin{array}{l}\text { Self-reported } \\
\text { musculoskeletal complaints } \\
\text { since starting current job } \\
\text { (Chinese version Nordic } \\
\text { Questionnaire) in } \\
\text { - } \quad \text { Neck } \\
\text { - } \quad \text { Shoulder } \\
\text { - } \quad \text { Low-back } \\
\text { - } \quad \text { Lower leg } \\
\text { Prevalence: } \\
\text { - } \\
\text { - } \quad \text { Shoulders } 17.2 \% \\
\text { - } \quad \text { Low-back } 32.9 \% \\
\text { - } \quad \text { Lower legs } 22.3 \%\end{array}$ & $\begin{array}{ll}\text { - } & \text { Standing }<4 \mathrm{hrs} / \text { day } \\
\text { - } & \text { Standing } 4-6 \\
\text { hrs } / \text { day } \\
\text { - } & \text { Standing }>6 \mathrm{hrs} / \text { day }\end{array}$ & $\begin{array}{l}\text { Reference } \\
\text { Low-back pain } \\
\text { OR:1.31 [1.10 1.55 }]^{1} \\
\text { Lower leg pain } \\
\text { OR:1.59 [1.30 1.95 }]^{1} \\
\text { Low-back pain } \\
\text { OR:1.51 [1.24 1.85 }]^{1} \\
\text { Lower leg pain } \\
\text { OR:1.73 [1.38 } 1.60]^{1}\end{array}$ & 0.95 \\
\hline $\begin{array}{l}\text { Jellad (2013) } \\
\text { [36] }\end{array}$ & - & Cross-sectional & $\begin{array}{l}\mathrm{n}=433 \\
\text { Females: } 55.9 \% \\
\text { Age: } 33.6 \text { years } \\
\text { Country: Tunisia } \\
\text { Occupation: } \\
\text { Hospital workers }\end{array}$ & - & & $\begin{array}{l}\text { Self-reported } \\
\text { prolonged standing } \\
\text { (frequency of standing; } \\
\text { not clear whether it is } \\
\text { just about } \\
\text { occupational standing) } \\
\text { classified into: } \\
\text { - Rarely: } \\
\quad \text { sometimes or } \\
\text { never } \\
\text { - Frequently: } \\
\text { often, always }\end{array}$ & $\begin{array}{l}\text { Self-reported } \\
\text { musculoskeletal symptoms } \\
\text { in last } 12 \text { months } \\
\text { Prevalence: } 65.4 \%\end{array}$ & $\begin{array}{ll}\text { - } & \text { Rarely standing } \\
\text { - } & \text { Frequently standing }\end{array}$ & $\begin{array}{l}74 \text { symptoms; } \\
25 \text { no symptoms } \\
209 \text { symptoms } \\
125 \text { no symptoms } \\
p=0.016 ;\end{array}$ & 0.64 \\
\hline $\begin{array}{l}\text { Jones (2007) } \\
\text { [37] }\end{array}$ & $\begin{array}{l}\text { The } \\
\text { New } \\
\text { Workers } \\
\text { Study }\end{array}$ & $\begin{array}{l}\text { Prospective (12 } \\
\text { and } 24 \text { month } \\
\text { follow-up). }\end{array}$ & $\begin{array}{l}\mathrm{n}=1198 \text { (671 } \\
\text { without knee pain } \\
\text { at baseline } \\
\text { combined with } 518 \\
\text { participants without } \\
\text { knee pain at } 12 \\
\text { months - } \\
\text { overlapping } \\
\end{array}$ & 1. & $\begin{array}{l}\text { Multivariate } \\
\text { model: age, } \\
\text { gender, } \\
\text { occupational } \\
\text { group; BMI } \\
\text { and physical } \\
\text { activity }\end{array}$ & $\begin{array}{l}\text { Self-reported duration } \\
\text { of occupational } \\
\text { standing, classified } \\
\text { into: } \\
\text { - None } \\
\text { - Standing <15 } \\
\text { mins in the last } \\
\quad \text { work day }\end{array}$ & $\begin{array}{l}\text { Self-reported knee pain } \\
\text { (lasting } \geq 1 \text { day in last } \\
\text { month), assessed at } \\
\text { baseline and during the } 12 \\
\text { and } 24 \text { month follow-up. } \\
\text { Baseline prevalence } 0 \% \text {. }\end{array}$ & $\begin{array}{l}\text { - } \quad \text { No standing } \\
12 \text { months ( } n=61 \\
\text { no pain, } n=4 \text { pain) } \\
24 \text { months }(n=50 \\
\text { no pain, } n=7 \text { pain) } \\
\begin{array}{l}\text { Standing }<15 \\
\text { mins } / \text { day }\end{array} \\
\end{array}$ & $\begin{array}{l}\text { Reference } \\
\text { RR: } 1.3\left[\begin{array}{ll}0.7 & 2.6\end{array}\right]^{1}\end{array}$ & 0.91 \\
\hline
\end{tabular}




\begin{tabular}{|c|c|c|c|c|c|c|c|c|}
\hline & & $\begin{array}{l}\text { numbers) } \\
\text { Females: } 35.8 \% \\
\text { Age: - } \\
\text { Country: England } \\
\text { Occupation: Newly } \\
\text { employed workers } \\
\text { from } 12 \text { diverse } \\
\text { occupational groups }\end{array}$ & & $\begin{array}{ll}- & \text { Standing }>15 \\
\text { mins in the last } \\
\text { work day }\end{array}$ & $\begin{array}{l}\text { Incidence: } 12 \text { months } 8.2 \% \text {; } \\
24 \text { months } 10.2 \%\end{array}$ & $\begin{array}{l}12 \text { months }(\mathrm{n}=171 \\
\text { no pain, } \mathrm{n}=15 \text { pain) } \\
24 \text { months }(\mathrm{n}=157 \\
\text { no pain, } \mathrm{n}=18 \text { pain) } \\
\\
\text { Standing }>15 \\
\text { mins/day } \\
24 \text { month ( } \mathrm{n}=381 \\
\text { no pain, } \mathrm{n}=36 \text { pain) } \\
24 \text { months ( } \mathrm{n}=251 \\
\text { no pain, } \mathrm{n}=27 \text { pain) }\end{array}$ & RR: $1.2\left[\begin{array}{ll}0.6 & 2.3\end{array}\right]^{1}$ & \\
\hline $\begin{array}{l}\text { Kaneda } \\
\text { (2001) [38] }\end{array}$ & Cross-sectional & $\begin{array}{l}\mathrm{n}=19,948 \\
\text { Females:- } \\
\text { Age: } 42.0 \text { (12.3) } \\
\text { years (low-back } \\
\text { pain); } 40.9 \text { (13.62) } \\
\text { years (no low-back } \\
\text { pain) } \\
\text { Country: Japan } \\
\text { Occupation: } \\
\text { Construction } \\
\text { workers }\end{array}$ & $\begin{array}{ll}\text { 1. } & \text { Univariate } \\
\text { model } \\
\text { 2. } \\
\text { Multi-variate } \\
\text { model: All } \\
\text { studied } \\
\text { personal } \\
\text { factors and } \\
\text { physical work } \\
\text { demands. }\end{array}$ & $\begin{array}{l}\text { Self-reported } \\
\text { occupational standing } \\
\text { (posture often } \\
\text { maintain at work) }\end{array}$ & $\begin{array}{l}\text { Self-reported severity of } \\
\text { low-back pain at the time of } \\
\text { the survey, past history of } \\
\text { low-back pain, progression, } \\
\text { severity, relationship to } \\
\text { work, onset. Definition of } \\
\text { cases and controls is } \\
\text { unclear. } \\
\text { Prevalence: } 29.3 \%\end{array}$ & $\begin{array}{ll}- & \begin{array}{l}\text { Sitting often } \\
\text { maintained }\end{array} \\
\text { - } & \begin{array}{l}\text { Standing often } \\
\text { maintained }\end{array} \\
\end{array}$ & $\begin{array}{l}\text { Reference } \\
\text { OR:0.83 }\left[\begin{array}{ll}0.75 & 0.92\end{array}\right]^{1}\end{array}$ & 0.68 \\
\hline $\begin{array}{l}\text { Karahan } \\
\text { (2009) [39] }\end{array}$ & Cross-sectional & $\begin{array}{l}\mathrm{n}=1600 \\
\text { Females: } 68.6 \% \\
\text { Age: } 28.02(5.0) \\
\text { years } \\
\text { Country: Turkey } \\
\text { Occupation: Staff } \\
\text { from } 6 \text { different } \\
\text { hospitals }\end{array}$ & $\begin{array}{ll}\text { 1. } & \text { Univariate } \\
\text { 2. Multivariate } \\
\text { model: age, } \\
\text { gender, } \\
\text { occupation, } \\
\text { education } \\
\text { status, years } \\
\text { worked, BMI, } \\
\text { smoking } \\
\text { behaviour, } \\
\text { exercise } \\
\text { patterns, } \\
\text { perceived } \\
\text { stress, lifting, } \\
\text { supporting, } \\
\text { pulling/positi } \\
\text { oning heavy } \\
\text { objects }\end{array}$ & $\begin{array}{l}\text { Self-reported total } \\
\text { duration of } \\
\text { occupational standing } \\
\text { time in a typical day }\end{array}$ & $\begin{array}{l}\text { Self-reported low-back pain } \\
\text { for at least } 1 \text { day during the } \\
\text { last } 12 \text { months } \\
\text { Prevalence: } 65.8 \%\end{array}$ & 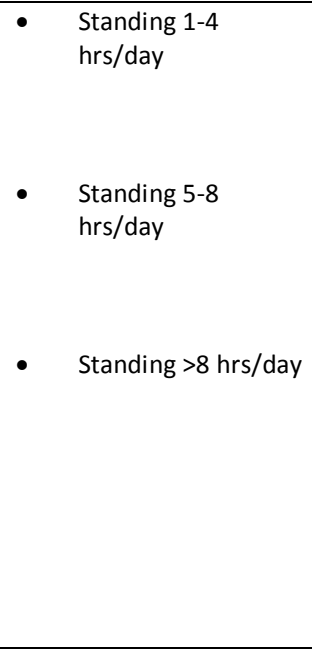 & $\begin{array}{l}\text { 166/301 }(55.1 \%) \text { low- } \\
\text { back pain; } 135 / 301 \\
(44.9 \%) \text { no low-back } \\
\text { pain } \\
498 / 772(64.5 \%) \text { low- } \\
\text { back pain; } 274 / 772 \\
(35.5 \%) \text { no low-back } \\
\text { pain } \\
388 / 527(73.6 \%) \text { low- } \\
\text { back pain; } 139 / 527 \\
(26.4 \%) \text { no low-back } \\
\text { pain } \\
\text { p <0.001 }\end{array}$ & 0.95 \\
\hline
\end{tabular}




\begin{tabular}{|c|c|c|c|c|c|c|c|c|c|}
\hline & & & & & & & & $\begin{array}{l}\text { - standing not } \\
\text { significantly } \\
\text { associated with low- } \\
\text { back pain (ORs not } \\
\text { reported) }\end{array}$ & \\
\hline $\begin{array}{l}\text { Kulcu (2010) } \\
{[40]}\end{array}$ & & Cross-sectional & $\begin{array}{l}\mathrm{n}=206 \\
\text { Females: } 57 \% \\
\text { Age: } 23.5(5.0) \\
\text { years } \\
\text { Country: Turkey } \\
\text { Occupation: } \\
\text { Dentists, students } \\
\text { and nurses without } \\
\text { back/neck } \\
\text { symptoms. }\end{array}$ & & $\begin{array}{l}\text { Self-reported duration } \\
\text { of occupational } \\
\text { standing (mins/day) }\end{array}$ & $\begin{array}{l}\text { Self-reported experience of } \\
\text { low-back and neck pain in } \\
\text { specific positions. Neck } \\
\text { Disability Index (NPDI), } \\
\text { Roland-Morris low-back } \\
\text { pain and Disability } \\
\text { Questionnaire (RMQ) } \\
\text { Prevalence: } \\
\text { - Low-back pain } 61 \% \\
\text { - Neck pain } 34 \%\end{array}$ & $\begin{array}{l}\text { Average duration of work } \\
\text { while standing } 73.4(14.3) \\
\text { mins/day }\end{array}$ & $\begin{array}{l}\text { Correlation between } \\
\text { working while } \\
\text { standing and } \\
\text { RMQ: } r=0.252 \text {, } \\
p<0.001 \\
\text { NPDI: } r=0.334 \text {, } \\
p<0.001 \\
\text { Neck pain (VAS) } \\
r=0.279, p<0.001 \\
\text { Low-back pain (VAS) } \\
r=0.200, p=0.005 \\
\text { Frequency of low- } \\
\text { back pain while } \\
\text { standing: } r=0.310, \\
p<0.001\end{array}$ & 0.73 \\
\hline $\begin{array}{l}\text { Lehto (1991) } \\
\text { [41] }\end{array}$ & - & Cross-sectional & $\begin{array}{l}\mathrm{n}=131 \\
\text { Females: } 68 \% \\
\text { Age: } 46 \text { [33-65] } \\
\text { years } \\
\text { Country: Finland } \\
\text { Occupation: } \\
\text { Dentists }\end{array}$ & $\begin{array}{l}\text { 1. } \begin{array}{l}\text { Multivariate } \\
\text { model: age, } \\
\text { gender. }\end{array} \\
\end{array}$ & $\begin{array}{l}\text { Self-reported } \\
\text { occupational standing }\end{array}$ & 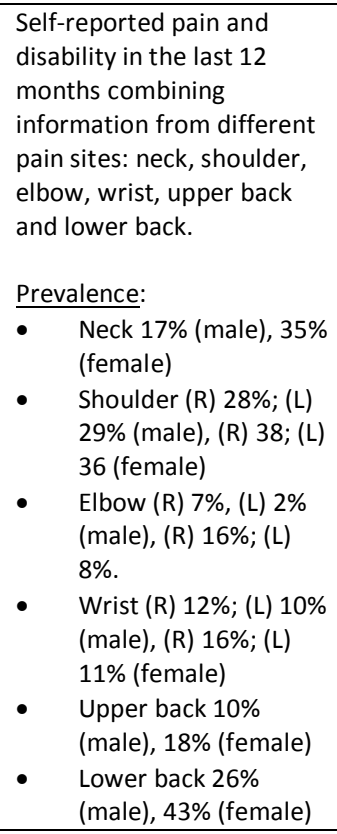 & $\begin{array}{ll}\text { - } & \text { Working in sitting } \\
\text { posture }\end{array}$ & $\begin{array}{l}\text { 58\% pain and } \\
\text { disability } \\
41 \% \text { pain and } \\
\text { disability } \\
\text { No statistically } \\
\text { significant differences }\end{array}$ & 0.68 \\
\hline Leino (1999) & - & Cross-sectional & $n=85$ & - & Self-reported & Self-reported discomfort & Standing & $33.7 \%$ & 0.50 \\
\hline
\end{tabular}




\begin{tabular}{|c|c|c|c|c|c|c|c|c|c|}
\hline [42] & & & $\begin{array}{l}\text { Females: } 95.3 \% \\
\text { Age: } 36 \text { years (small } \\
\text { salons); } 29 \text { years } \\
\text { (large salons) } \\
\text { Country: Finland } \\
\text { Occupation: } \\
\text { Hairdressers }\end{array}$ & & occupational standing & $\begin{array}{l}\text { from work factors } \\
\text { Prevalence: - }\end{array}$ & & $\begin{array}{l}\text { No effect } \\
55.4 \% \\
\text { Some discomfort } \\
9.6 \% \\
\text { A lot of discomfort } \\
1.2 \% \text { Cause a disease }\end{array}$ & \\
\hline $\begin{array}{l}\text { Leroux } \\
\text { (2005) [43] }\end{array}$ & $\begin{array}{l}\text { Quebec } \\
\text { Health } \\
\text { Study }\end{array}$ & Cross-sectional & $\begin{array}{l}\mathrm{n}=9496 \\
\text { Females: } 42 \% \\
\text { Age: } 18-65 \text { years } \\
\text { Country: Canada } \\
\text { Occupation: All } \\
\text { people living in } \\
\text { private households } \\
\text { with a paid } \\
\text { occupation }\end{array}$ & $\begin{array}{l}\text { Stratified by } \\
\text { gender. } \\
\text { 1. Multivariate } \\
\text { model: age, } \\
\text { psychological } \\
\text { distress, } \\
\text { smoking, } \\
\text { traumatic } \\
\text { events and } \\
\text { work factors; } \\
\text { and variables } \\
\text { significantly } \\
\text { associated } \\
\text { with } \\
\text { musculoskele } \\
\text { tal pain }\end{array}$ & $\begin{array}{l}\text { Self-reported } \\
\text { occupational standing } \\
\text { (standing/sitting most } \\
\text { of the time at work) }\end{array}$ & 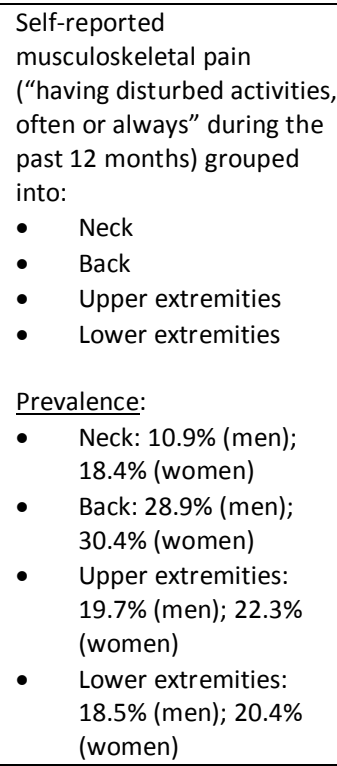 & $\begin{array}{l}\text { - } \begin{array}{l}\text { Sitting most of the } \\
\text { time at work } \\
\text { - Standing most of } \\
\text { the time at work }\end{array} \\
\end{array}$ & $\begin{array}{l}\text { Reference } \\
\text { Lower extremities } \\
\text { Men } \\
\text { OR: } 2.4\left[\begin{array}{ll}1.8 & 3.1\end{array}\right]^{1} \\
\text { Women } \\
\text { OR: } 2.9\left[\begin{array}{ll}2.1 & 3.9\end{array}\right]^{1}\end{array}$ & 0.95 \\
\hline $\begin{array}{l}\text { Levangie } \\
\text { (1999) [44] }\end{array}$ & - & Case-control & $\begin{array}{l}\mathrm{n}=283 \\
\text { Female: } 60 \% \\
\text { (cases), } 61 \% \\
\text { (controls) } \\
\text { Age: } 35.2 \text { years } \\
\text { (cases), } 35.5 \text { years } \\
\text { (controls) } \\
\text { Country: USA }\end{array}$ & - & $\begin{array}{l}\text { Self-reported standing } \\
\text { (not clear whether it is } \\
\text { just about } \\
\text { occupational standing; } \\
\text { duration) }\end{array}$ & $\begin{array}{l}\text { Self-reported low-back pain } \\
\text { with cases (seeking } \\
\text { treatment of low-back pain, } \\
\leq 1 \text { year in duration) and } \\
\text { controls (patients treated by } \\
\text { a physical therapist for an } \\
\text { upper extremity problem } \\
\text { that was not obviously neck } \\
\text { or back-related) } \\
\text { Prevalence: } 52.1 \%(150 \\
\text { cases, } 138 \text { controls) }\end{array}$ & 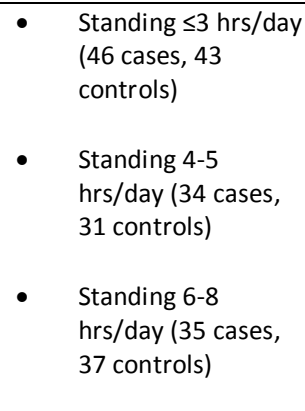 & $\begin{array}{l}\text { Reference } \\
\text { OR:1.03 [0.54 1.94] } \\
\text { OR:0.88 [0.47 1.65] }\end{array}$ & 0.73 \\
\hline
\end{tabular}




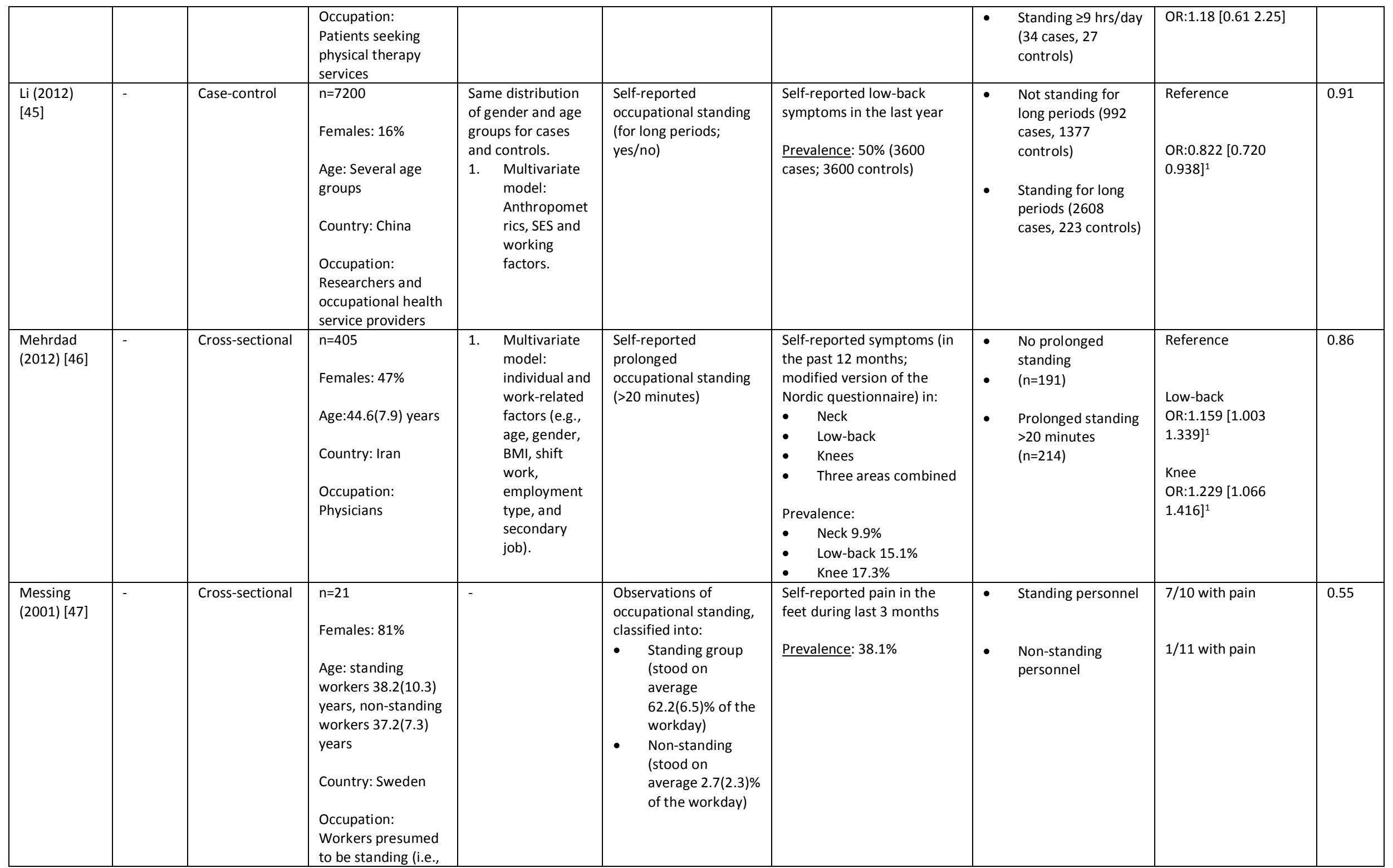




\begin{tabular}{|c|c|c|c|c|c|c|c|c|c|}
\hline & & & $\begin{array}{l}\text { works from } \\
\text { department store } \\
\text { and a restaurant) } \\
\text { and non-standing } \\
\text { workers (i.e., } \\
\text { research personnel) }\end{array}$ & & & & & & \\
\hline $\begin{array}{l}\text { Messing } \\
\text { (2008) [48] }\end{array}$ & $\begin{array}{l}\text { Quebec } \\
\text { Health } \\
\text { and } \\
\text { Social } \\
\text { Study }\end{array}$ & Cross-sectional & $\begin{array}{l}\mathrm{n}=7757 \\
\text { Females:41\% } \\
\text { Age: } 18-65 \text { years. } \\
\text { Country: Canada } \\
\text { Occupation: } \\
\text { General population }\end{array}$ & $\begin{array}{l}\text { Stratified by } \\
\text { gender. } \\
\text { 1. Multivariate } \\
\text { model: age, } \\
\text { BMI, job } \\
\text { strain and } \\
\text { other physical } \\
\text { work } \\
\text { demands. }\end{array}$ & $\begin{array}{l}\text { Self-reported } \\
\text { occupational standing. } \\
\text { Participants who } \\
\text { reported on standing } \\
\text { most of the timey were } \\
\text { questioned on the } \\
\text { nature of their } \\
\text { standing postures. }\end{array}$ & $\begin{array}{l}\text { Self-reported (adapted } \\
\text { Nordic questionnaire) } \\
\text { significant pain in a lower } \\
\text { extremities during the past } \\
12 \text { months, interfering with } \\
\text { usual activities in the } \\
\text { following areas: } \\
\text { - } \quad \text { Lower legs/calves } \\
\text { - } \quad \text { Ankles / feet } \\
\text { - } \quad \text { Knees } \\
\text { - } \quad \text { Hips or thighs } \\
\text { Prevalence } \\
-\quad \text { Lower legs/calves } \\
\text { - } \quad \text { Ankles / feet } 9.4 \% \\
\text { - Knees } 8.6 \% \\
\text { - Hips or thighs 5.0\% }\end{array}$ & $\begin{array}{l}\text { - } \begin{array}{l}\text { Sitting with the } \\
\text { possibility of } \\
\text { getting up at will } \\
\text { - Standing with the } \\
\text { possibility of sitting } \\
\text { down at will }\end{array} \\
\\
\text { Standing in a } \\
\text { fixed or } \\
\text { relatively } \\
\text { fixed position }\end{array}$ & 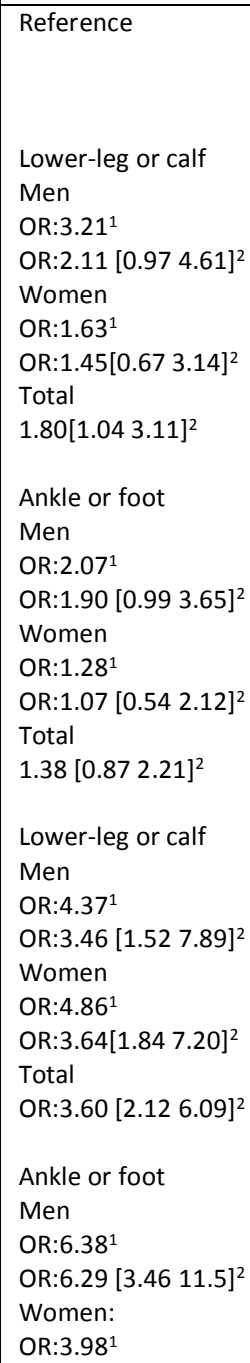 & 0.95 \\
\hline
\end{tabular}




\begin{tabular}{|c|c|c|c|c|c|c|c|c|c|}
\hline & & & & & & & $\begin{array}{l}\text { - Usual posture } \\
\text { sitting } \\
\text { - Usual posture } \\
\text { standing }\end{array}$ & $\begin{array}{l}\text { OR:2.78 [1.49 } 5.21]^{2} \\
\text { Total } \\
\text { OR:3.95 [2.56 6.10 }]^{2} \\
\text { Reference } \\
\\
\text { Lower-leg or calf pain } \\
\text { Men } \\
\text { OR:2.851 } \\
\text { Women } \\
\text { 3.72 }{ }^{1} \\
\text { Ankle or foot pain } \\
\text { Men } \\
\text { OR:2.81 } \\
\text { Women } \\
\text { OR:3.61 }\end{array}$ & \\
\hline $\begin{array}{l}\text { Mohseni- } \\
\text { Banpei } \\
\text { (2011) [49] }\end{array}$ & - & Cross-sectional & $\begin{array}{l}\mathrm{n}=223 \\
\text { Females: } 51.6 \% \\
\text { Age: } 42.66(7.51) \\
\text { years. } \\
\text { Country: Iran } \\
\text { Occupation: } \\
\text { Surgeons }\end{array}$ & - & $\begin{array}{l}\text { Self-reported } \\
\text { occupational } \\
\text { prolonged standing }\end{array}$ & $\begin{array}{l}\text { Self-reported low-back pain } \\
\text { (visual analog scale with } 0 \\
\mathrm{~mm} \text { indicating no pain and } \\
100 \mathrm{~mm} \text { indicating } \\
\text { unbearable pain). Definition } \\
\text { of cases and controls is not } \\
\text { specified. } \\
\text { Prevalence: } \\
\text { - } \quad \text { Currently } 39.9 \% \\
\text { - Last month } 50.2 \% \\
\text { - } \quad \text { Six month } 62.3 \% \\
\text { - } \text { Annual } 71.7 \% \\
\text { - } \quad \text { Lifetime } 84.8 \%\end{array}$ & & $\begin{array}{l}\text { Prolonged standing, } \\
\text { was the most } \\
\text { significant } \\
\text { aggravating factors: } \\
(85.2 \%)\end{array}$ & 0.68 \\
\hline $\begin{array}{l}\text { Nahit (2001) } \\
\text { [50] }\end{array}$ & - & Cross-sectional & $\begin{array}{l}\mathrm{n}=1081 \\
\text { Females: } 32 \% \\
\text { Age: } 23 \text { (median) } \\
\text { years } \\
\text { Country: UK } \\
\text { Occupation: Range } \\
\text { of occupations and } \\
\text { industries with }\end{array}$ & $\begin{array}{l}\text { Multivariate } \\
\text { model: age } \\
\text { and gender. }\end{array}$ & $\begin{array}{l}\text { Self-reported } \\
\text { occupational standing } \\
\text { (duration), classified } \\
\text { into } \\
\text { - } \quad<15 \text { minutes/day } \\
\text { - } \quad 15 \text { minutes to }<2 \\
\quad \text { hrs/day } \\
\text { - } \quad 2 \text { hrs to }<4 \\
\quad \text { hrs/day } \\
\text { - } \quad \geq 4 \text { hrs/day. }\end{array}$ & $\begin{array}{l}\text { Self-reported pain } \\
\text { experienced in the past } \\
\text { month lasting longer than } \\
24 \text { hours, in the following } \\
\text { areas: } \\
\text { - } \quad \text { Low-back } \\
\text { - Shoulder } \\
\text { - Wrist/forearm } \\
\text { - Knee } \\
\text { Prevalence: }\end{array}$ & $\begin{array}{ll}\text { - } & \text { Not standing } \geq 4 \\
\text { hrs/day ( } n=880, \\
n=205 \text { with pain) } \\
\\
\text { - Standing } \geq 4 \text { hrs/day } \\
\text { ( } \mathrm{n}=192, \mathrm{n}=55 \text { with } \\
\text { pain) } \\
\end{array}$ & $\begin{array}{l}\text { Reference } \\
\text { Low-back } \\
\text { OR:1.4 [0.97 2.0 }]^{1}\end{array}$ & 0.91 \\
\hline
\end{tabular}




\begin{tabular}{|c|c|c|c|c|c|c|c|c|c|}
\hline & & & $\begin{array}{l}\text { presumed high } \\
\text { prevalence of } \\
\text { musculoskeletal } \\
\text { symptoms. }\end{array}$ & & & $\begin{array}{ll} & \text { Low-back } 24.1 \% \\
- & \text { Shoulder } 20.4 \% \\
\text { - } & \text { Wrist/forearm } 8.6 \% \\
\text { - } & \text { Knee } 20.5 \%\end{array}$ & & & \\
\hline $\begin{array}{l}\text { Phajan } \\
\text { (2014) [51] }\end{array}$ & - & Cross-sectional & $\begin{array}{l}\mathrm{n}=540 \\
\text { Females: } 57.59 \% \\
\text { Age: } 44.75(7.67) \\
\text { years } \\
\text { Country: Thailand } \\
\text { Occupation: } \\
\text { Sugarcane farmers }\end{array}$ & - & $\begin{array}{l}\text { Self-reported duration } \\
\text { of standing (not clear } \\
\text { whether it is just about } \\
\text { occupational standing) }\end{array}$ & $\begin{array}{l}\text { Self-reported } \\
\text { musculoskeletal pain } \\
\text { (Nordic questionnaire) in } \\
\text { the last } 12 \text { months in any of } \\
\text { the following regions neck, } \\
\text { shoulder, elbow, wrist, } \\
\text { upper back, low-back, hip, } \\
\text { knee, ankle, and foot. } \\
\text { Prevalence: } 88.70 \%\end{array}$ & 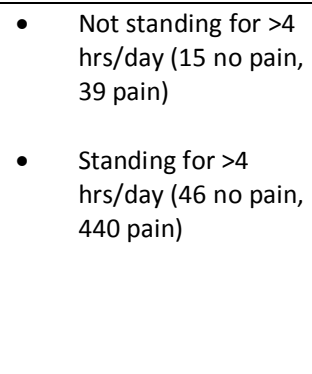 & $\begin{array}{l}\text { Reference } \\
\text { OR: } 3.67 \text { [1.88 7.17] }\end{array}$ & 0.86 \\
\hline $\begin{array}{l}\text { Pope (2003) } \\
\text { [52] }\end{array}$ & - & Case-control & $\begin{array}{l}\mathrm{n}=3354 \\
\begin{array}{l}\text { Females: } 63,6 \% \\
\text { (cases), } 49,4 \% \\
\text { (controls) }\end{array} \\
\text { Age: }>18 \text { years } \\
\text { Country: UK } \\
\text { Occupation: } \\
\text { General population } \\
\text { of participants } \\
\text { recruited through } \\
\text { general practices }\end{array}$ & 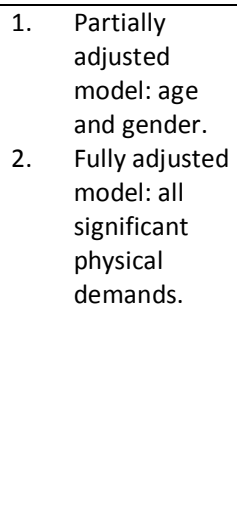 & $\begin{array}{l}\text { Self-reported history of } \\
\text { occupational standing } \\
\text { (in years). }\end{array}$ & $\begin{array}{l}\text { Self-reported hip pain in the } \\
\text { past month lasting at least } \\
24 \text { hours } \\
\text { Prevalence: } 10.5 \%\end{array}$ & 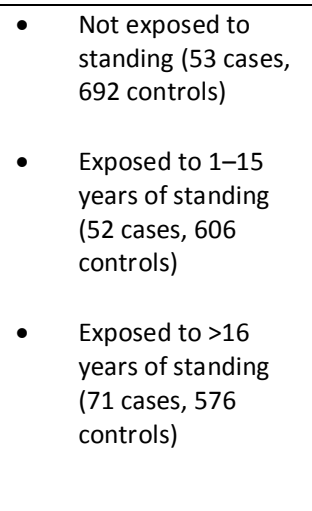 & $\begin{array}{l}\text { Reference } \\
\text { OR: } 1.19\left[\begin{array}{ll}0.80 & 1.78\end{array}\right]^{1} \\
\text { OR:1.46 [1.00 2.14 }{ }^{1}\end{array}$ & 0.91 \\
\hline $\begin{array}{l}\text { Rodigari } \\
\text { (2012) [53] }\end{array}$ & - & Cross-sectional & $\begin{array}{l}\mathrm{n}=100 \\
\text { Females: } 26 \% \\
\text { Age: } 40.1(10.85) \\
\text { years } \\
\text { Country: Italy } \\
\text { Occupation: Surgery } \\
\text { unit workers. }\end{array}$ & $\begin{array}{l}\text { Multivariate } \\
\text { model: body } \\
\text { measurement } \\
\text { s, working } \\
\text { activity, } \\
\text { fatigue and } \\
\text { pain, sporting } \\
\text { activities and } \\
\text { awareness of } \\
\text { guidelines. }\end{array}$ & $\begin{array}{l}\text { Self-reported } \\
\text { occupational standing } \\
\text { (mainly working in } \\
\text { sitting, standing or } \\
\text { alternating posture) }\end{array}$ & $\begin{array}{l}\text { Self-reported onset of pain } \\
\text { (visual-analog scale) in any } \\
\text { of the following areas: } \\
\text { shoulder, elbow, hands, } \\
\text { cervical spine, vertebral } \\
\text { column, lumbar spine, hips, } \\
\text { knees and feet. Definition of } \\
\text { cases and controls is } \\
\text { unclear. } \\
\text { Prevalence: } 70 \%\end{array}$ & $\begin{array}{ll}\text { - } & \text { Standing } \\
\text { - } & \text { Sitting } \\
\text { - } & \text { Alternate }\end{array}$ & $\begin{array}{l}\text { Pain: } 53(71.6 \%) \\
\text { No pain: } 21(28.4 \%) \\
\text { Pain: } 12(70.6 \%) \\
\text { No pain: } 5(29.3 \%) \\
\text { Pain: } 5(62.5 \%) \\
\text { No pain: } 3(37.5 \%) \\
\text { P=0.865 }\end{array}$ & 0.82 \\
\hline $\begin{array}{l}\text { Samad } \\
\text { (2010) [54] }\end{array}$ & - & Cross-sectional & $\begin{array}{l}\mathrm{n}=272 \\
\text { Females: } 67 \%\end{array}$ & - & $\begin{array}{l}\text { Self-reported } \\
\text { prolonged standing } \\
\text { contributing to pain } \\
\text { (not clear whether it is }\end{array}$ & $\begin{array}{l}\text { Self-reported low-back pain. } \\
\text { (Nordic questionnaire) in } \\
\text { the previous } 12 \text { months. }\end{array}$ & & $\begin{array}{l}\text { Prolonged standing } \\
\text { contributed in } 26 \\
(23.4 \%) \text { participants } \\
\text { to low-back pain. }\end{array}$ & 0.68 \\
\hline
\end{tabular}




\begin{tabular}{|c|c|c|c|c|c|c|c|c|c|}
\hline & & & $\begin{array}{l}\text { Age: } 34.74(7.7) \\
\text { years } \\
\text { Country: Malaysia } \\
\text { Occupation: } \\
\text { Teachers }\end{array}$ & & $\begin{array}{l}\text { just about } \\
\text { occupational standing) }\end{array}$ & Prevalence: $40.4 \%$ & & & \\
\hline $\begin{array}{l}\text { Sanya (2005) } \\
\text { [55] }\end{array}$ & - & Cross-sectional & $\begin{array}{l}\mathrm{n}=604 \\
\text { Females: } 14.7 \% \\
\text { Age: } 20-60 \text { years } \\
\text { Country: Nigeria } \\
\text { Occupation: Nine } \\
\text { different industries } \\
\text { (e.g., } \\
\text { manufacturing, } \\
\text { food and drink } \\
\text { processing } \\
\text { industries) }\end{array}$ & - & $\begin{array}{l}\text { Self-reported } \\
\text { occupational } \\
\text { prolonged standing }\end{array}$ & $\begin{array}{l}\text { Self-reported low-back pain. } \\
\text { Definition of cases and } \\
\text { controls is unclear. } \\
\text { Prevalence: } \\
\quad \text { Point } 59.7 \% \\
\text { - } 12 \text {-month } 59.5 \%\end{array}$ & & $\begin{array}{l}62.4 \% \text { of low-back } \\
\text { pain was caused by } \\
\text { prolonged standing } \\
(\mathrm{p}=0.61)\end{array}$ & 0.59 \\
\hline $\begin{array}{l}\text { Schierhout } \\
\text { (1995) [56] }\end{array}$ & - & Cross-sectional & $\begin{array}{l}\mathrm{n}=401 \\
\text { Females: } 62 \% \\
\text { Age: - } \\
\text { Country: South- } \\
\text { Africa } \\
\text { Occupation: Factory } \\
\text { workers }\end{array}$ & $\begin{array}{ll}\text { 1. } & \text { Univariate: } \\
& \text { stratified for } \\
& \text { gender. } \\
\text { 2. } & \text { Multivariate } \\
& \text { model: } \\
\text { gender, age, } \\
\text { height, all } \\
\text { work } \\
\text { demands }\end{array}$ & $\begin{array}{l}\text { Observations of } \\
\text { occupational standing }\end{array}$ & $\begin{array}{l}\text { Self-reported } \\
\text { musculoskeletal pain in any } \\
\text { site (symptoms that have } \\
\text { been developed since } \\
\text { working in the current job). } \\
\text { Authors distinguish } \\
\text { between acute and chronic } \\
\text { pain (the definition and } \\
\text { outcome used for analysis is } \\
\text { unclear). Analyses were } \\
\text { performed on: } \\
\text { - Neck/shoulder } \\
\text { - Low-back and } \\
\text { Prevalence: - }\end{array}$ & $\begin{array}{l}\text { Seated work } \\
\\
\text { Standing work } \\
(\mathrm{n}=225)\end{array}$ & $\begin{array}{l}\text { Neck/shoulder pain } \\
\text { Men } \\
\text { Prev:0.13 [0.000 } \\
0.294] \\
\text { Women } \\
\text { Prev:0.31 [0.188 } \\
0.432] \\
\text { Back pain } \\
\text { Men } \\
\text { Prev:0.13 [0.000 } \\
0.294] \\
\text { Women } \\
\text { Prev:0.23 [0.118 } \\
0.341] \\
\\
\text { Forearm/wrist/hand } \\
\text { Men } \\
\text { Prev:0.06 [0.015 } \\
0.105] \\
\text { Women } \\
\text { Prev:0.02 [0.009 } \\
0.031]\end{array}$ & 0.86 \\
\hline
\end{tabular}




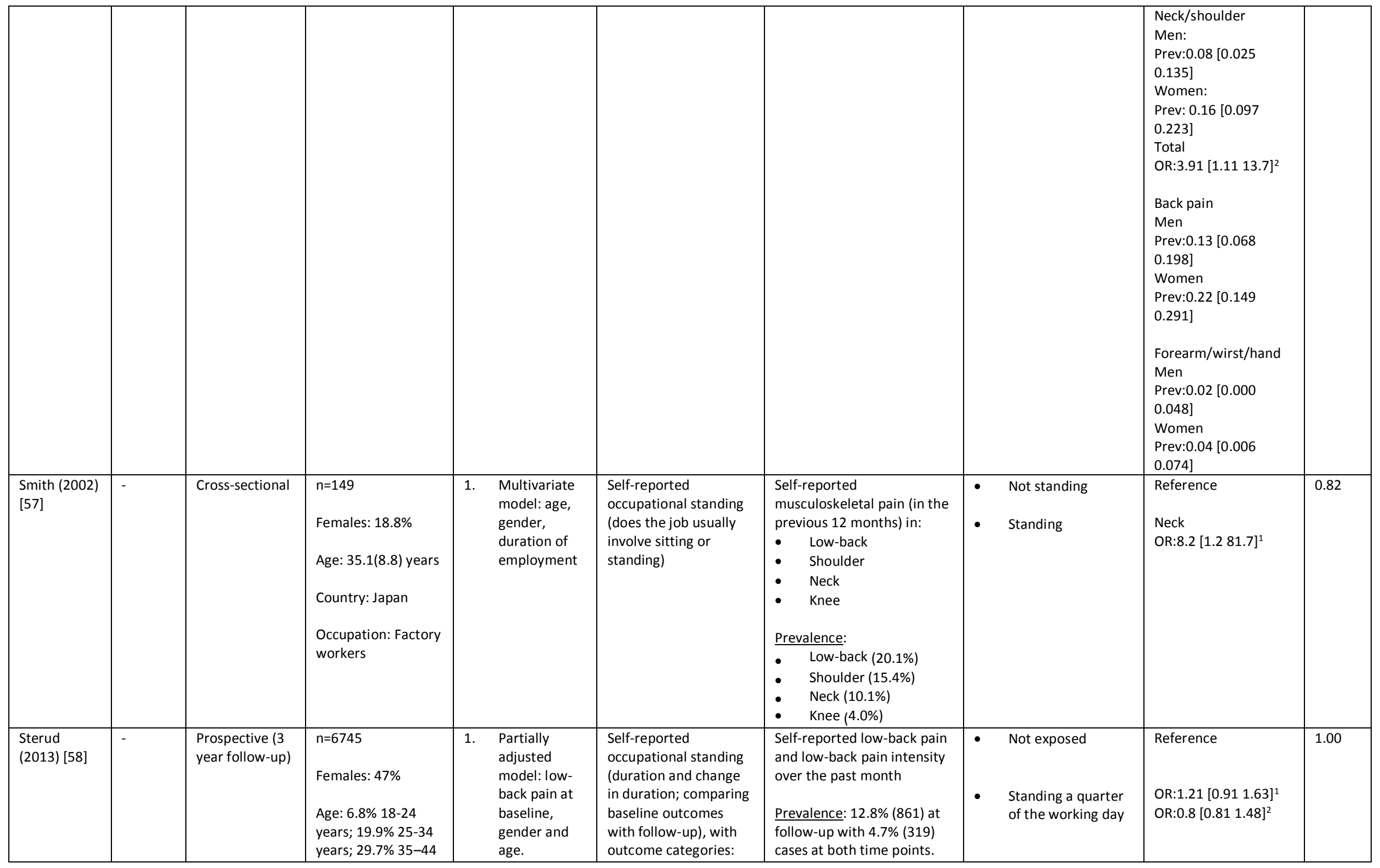




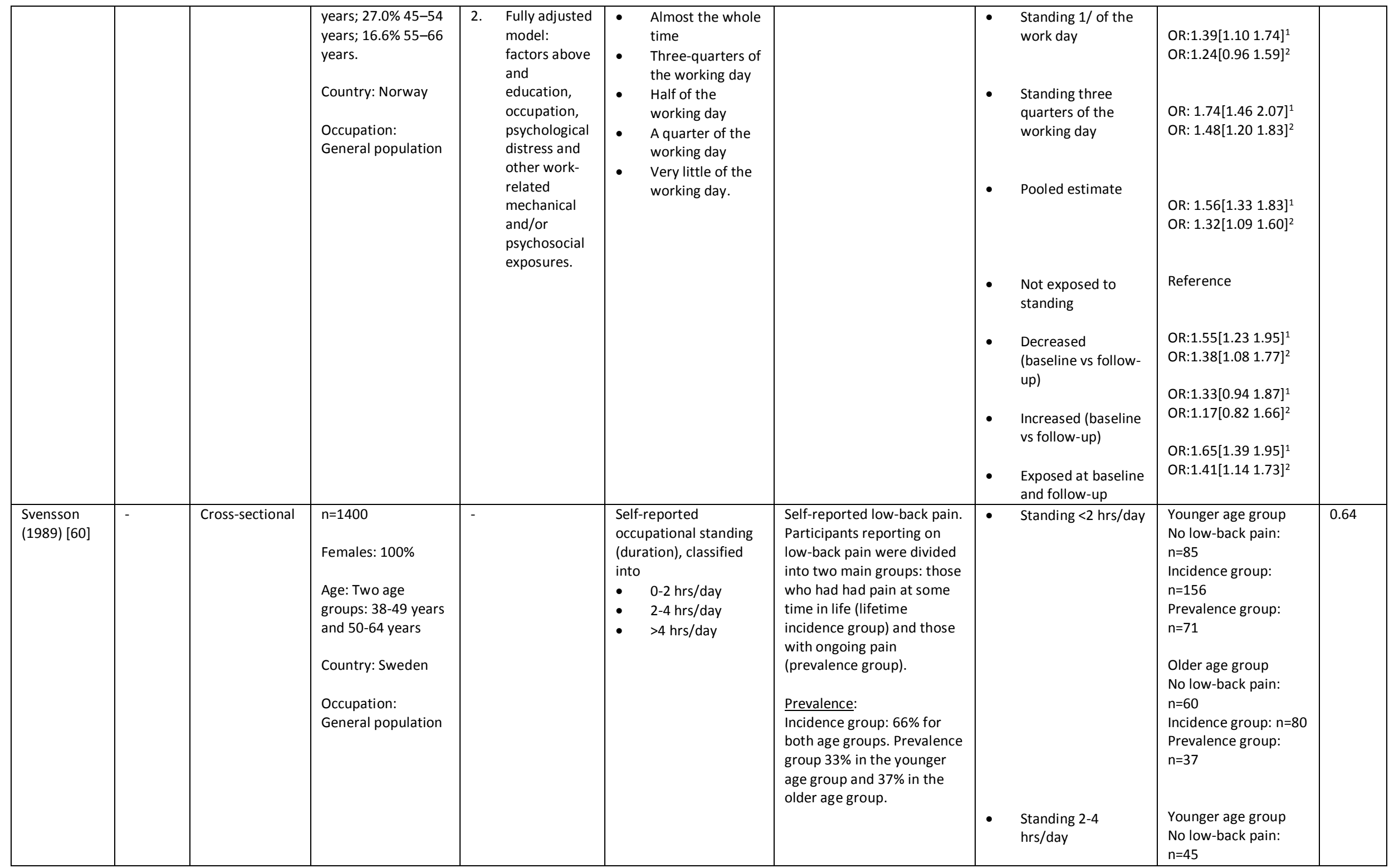




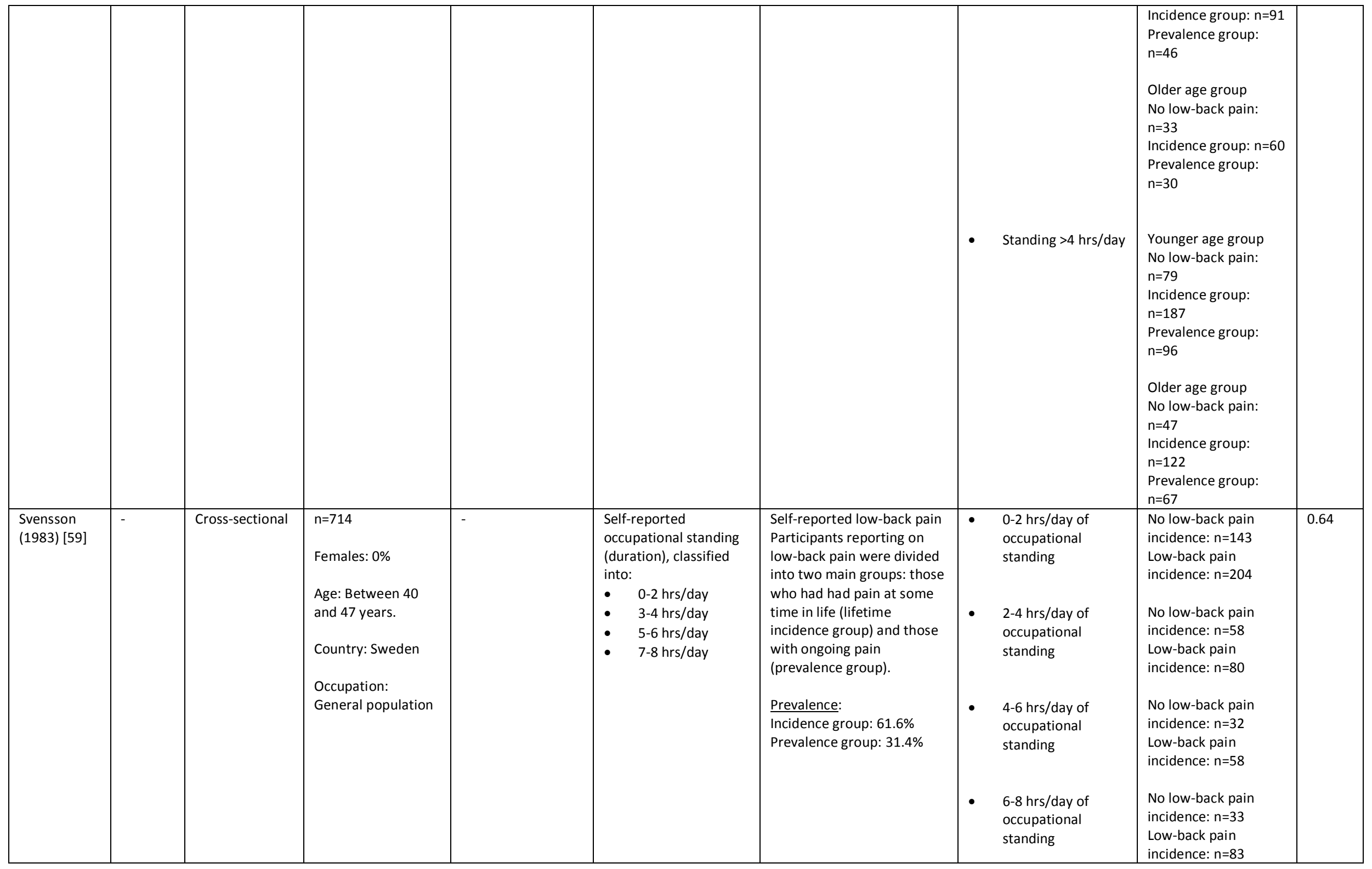




\begin{tabular}{|c|c|c|c|c|c|c|c|c|c|c|}
\hline & & & & & & & & & $P<0.05$ & \\
\hline $\begin{array}{l}\text { Tissot (2009) } \\
\text { [61] }\end{array}$ & $\begin{array}{l}\text { Quebec } \\
\text { Social } \\
\text { and } \\
\text { Health } \\
\text { Survey }\end{array}$ & Cross-sectional & $\begin{array}{l}\mathrm{n}=7730 \\
\text { Females: } 41.6 \% \\
\text { Age: } 18-65 \text { year } \\
\text { Country: Canada } \\
\text { Occupation: } \\
\text { General population }\end{array}$ & - & & $\begin{array}{l}\text { Self-reported } \\
\text { occupational standing. } \\
\text { Those who reported } \\
\text { that they usually stood } \\
\text { at work were } \\
\text { questioned on the } \\
\text { nature of their } \\
\text { standing postures }\end{array}$ & 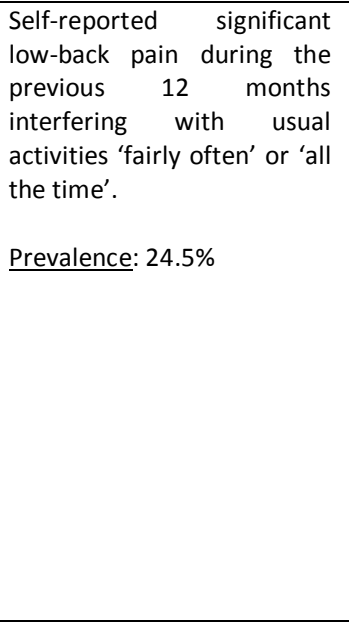 & 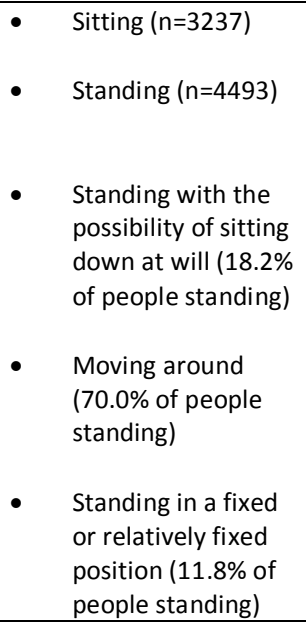 & $\begin{array}{l}\text { Low-back pain: } 28.6 \% \\
\text { Low-back pain: } 30.4 \%\end{array}$ & 0.77 \\
\hline $\begin{array}{l}\text { Tomita } \\
(2010) \text { [62] }\end{array}$ & - & Cross-sectional & $\begin{array}{l}\mathrm{n}=165 \\
\text { Females: } 68.5 \% \\
\text { Age: } 30.3(10.4) \\
\text { years } \\
\text { Country: Thailand } \\
\text { Occupation: } \\
\text { Seafood processing } \\
\text { industry workers }\end{array}$ & & $\begin{array}{l}\text { Multivariate } \\
\text { model: age } \\
\text { and gender }\end{array}$ & $\begin{array}{l}\text { Self-reported } \\
\text { occupational standing } \\
\text { (duration) categorised } \\
\text { into: } \\
\text { - Seldom/never } \\
\text { - Sometimes/most } \\
\quad \text { of the time }\end{array}$ & $\begin{array}{l}\text { Self-reported current low- } \\
\text { back pain } \\
\text { Prevalence: point } 28.5 \%\end{array}$ & $\begin{array}{l}\text { Standing seldom or } \\
\text { never ( } \mathrm{n}=20, \\
\text { prevalence } 10 \%) \\
\\
\quad \text { Standing } \\
\text { sometimes, often } \\
\text { or most of the time } \\
(\mathrm{n}=149, \text { prevalence } \\
30 \%)\end{array}$ & $\begin{array}{l}\text { Reference } \\
\text { OR:3.07 [0.65 14.41 }]^{1}\end{array}$ & 0.82 \\
\hline $\begin{array}{l}\text { Trinkoff } \\
\text { (2003) [63] }\end{array}$ & - & Cross-sectional & $\begin{array}{l}\mathrm{n}=1163 \\
\text { Females: } 94 \% \\
\text { Age: } 45 \text { years } \\
\text { Country: USA } \\
\text { Occupation: Nurses }\end{array}$ & & $\begin{array}{l}\text { Multivariate } \\
\text { model: age }\end{array}$ & $\begin{array}{l}\text { Self-reported } \\
\text { occupational } \\
\text { prolonged standing in } \\
\text { one place/static } \\
\text { position ( }>30 \text { minutes) }\end{array}$ & $\begin{array}{l}\text { Self-reported } \\
\text { musculoskeletal symptoms } \\
\text { in the past year that lasted } \\
>1 \text { week, or occurred at } \\
\text { least monthly in: } \\
\text { - Neck } \\
\text { - Shoulder } \\
\text { - Back } \\
\text { With at least moderate pain } \\
\text { on average (based on a } 5 \text { - } \\
\text { point pain scale). Non- } \\
\text { work-related injuries were } \\
\text { excluded. }\end{array}$ & 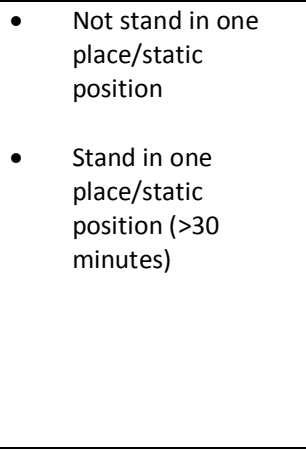 & 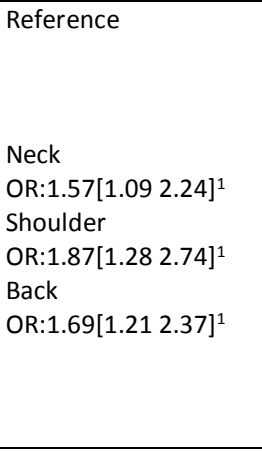 & 0.77 \\
\hline
\end{tabular}




\begin{tabular}{|c|c|c|c|c|c|c|c|c|c|}
\hline & & & & & & Prevalence:- & & & \\
\hline $\begin{array}{l}\text { Vahdati } \\
\text { (2014) [64] }\end{array}$ & - & Cross-sectional & $\begin{array}{l}\mathrm{n}=125 \\
\text { Females: } 41.6 \% \\
\text { Age: } 33.6(4.81) \\
\text { years } \\
\text { Country: Iran } \\
\text { Occupation: } \\
\text { Medical residents }\end{array}$ & - & $\begin{array}{l}\text { Self-reported } \\
\text { occupational standing }\end{array}$ & $\begin{array}{l}\text { Self-reported low-back pain } \\
\text { during the past } 12 \text { months. } \\
\text { Prevalence: } 56.8 \%\end{array}$ & $\begin{array}{l}\text { Not standing for a } \\
\text { long time ( } \mathrm{n}=12,11 \\
\text { with low-back pain) } \\
\text { - } \quad \text { Standing for a long } \\
\text { time ( } \mathrm{n}=113,43 \\
\text { with low-back pain) }\end{array}$ & $\begin{array}{l}\text { Standing for long } \\
\text { periods was } \\
\text { significantly } \\
\text { associated with low- } \\
\text { back pain }\end{array}$ & 0.73 \\
\hline $\begin{array}{l}\text { Xiao (2013) } \\
\text { [65] }\end{array}$ & $\begin{array}{l}\text { MICASA } \\
\text { study }\end{array}$ & Cross-sectional & $\begin{array}{l}\mathrm{n}=759 \\
\text { Females: } 44.7 \% \\
\text { Age: } 37.9 \text { (median) } \\
\text { years } \\
\text { Country: USA } \\
\text { Occupation: Farm } \\
\text { workers }\end{array}$ & $\begin{array}{l}\text { Stratified by } \\
\text { gender. } \\
1 \quad \text { Multivariate } \\
\text { model: age, } \\
\text { smoking } \\
\text { status and } \\
\text { years working } \\
\text { in agriculture. }\end{array}$ & $\begin{array}{l}\text { Self-reported duration } \\
\text { of occupational } \\
\text { standing }\end{array}$ & $\begin{array}{l}\text { Self-reported } \\
\text { musculoskeletal pain over } \\
\text { the last } 12 \text { months at six } \\
\text { sites: } \\
\text { - } \quad \text { Low-back, } \\
\text { - } \quad \text { Knee } \\
\text { - } \quad \text { Neck } \\
\text { - Finger } \\
\text { Those who answered yes } \\
\text { were if the pain lasted for } \\
\geq 6 \text { weeks and chronic pain } \\
\text { at each site was defined as } \\
\text { pain lasting six weeks or } \\
\text { longer } \\
\text { Prevalence: } \\
\text { - } \quad \text { Low-back pain: } 24.5 \% \\
\text { - Knee pain: } 13.7 \% \\
\text { - Hip pain: } 12.1 \% \\
\text { - Neck pain } 8.9 \% \\
\text { - Hand pain } 7.4 \% \\
\quad \text { Finger pain: } 7.1 \%\end{array}$ & 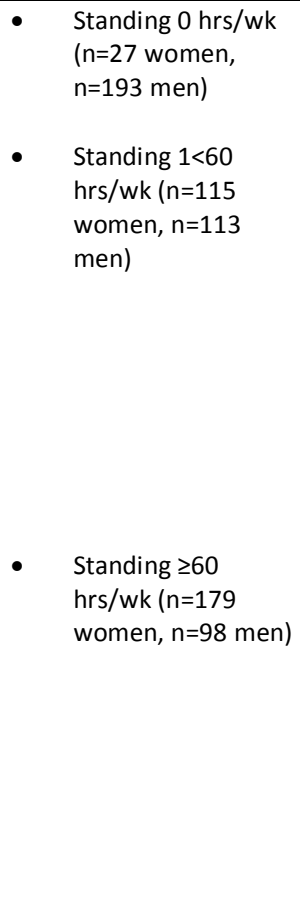 & 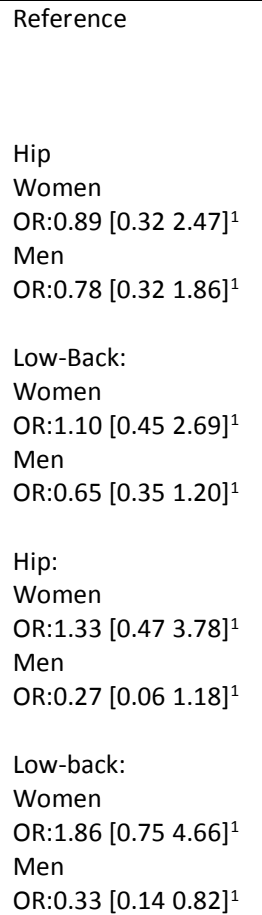 & 1.00 \\
\hline $\begin{array}{l}\mathrm{Xu}(1997) \\
{[66]}\end{array}$ & - & Cross-sectional & $\begin{array}{l}\mathrm{n}=5185 \\
\text { Females: - } \\
\text { Age: - }\end{array}$ & $\begin{array}{ll}1 & \text { Partially } \\
& \text { adjusted } \\
\text { model: sex, } \\
\text { age, } \\
\text { educational, } \\
\text { and }\end{array}$ & $\begin{array}{l}\text { Self-reported } \\
\text { occupational standing } \\
\text { (duration), classified } \\
\text { into: } \\
\text { - All of the time } \\
\text { - } \quad 3 / 4 \text { of the time }\end{array}$ & $\begin{array}{l}\text { Self-reported low-back pain } \\
\text { in the past } 12 \text { months. } \\
\text { Symptoms were defined as } \\
\text { all conditions of pain, ache, } \\
\text { or discomfort localised in } \\
\text { the lower back, regardless }\end{array}$ & $\begin{array}{l}\text { Never or seldom } \\
\text { standing ( } n=1363, \\
499 \text { with pain) } \\
\\
\text { - Standing at least } 1 / 4 \\
\text { of the working time }\end{array}$ & $\begin{array}{l}\text { Reference } \\
\text { OR: } 1.18^{2}, p=0.058 \\
\text { OR: } 1.20\left[\begin{array}{ll}1.04 & 1.38\end{array}\right]^{1}\end{array}$ & 0.82 \\
\hline
\end{tabular}




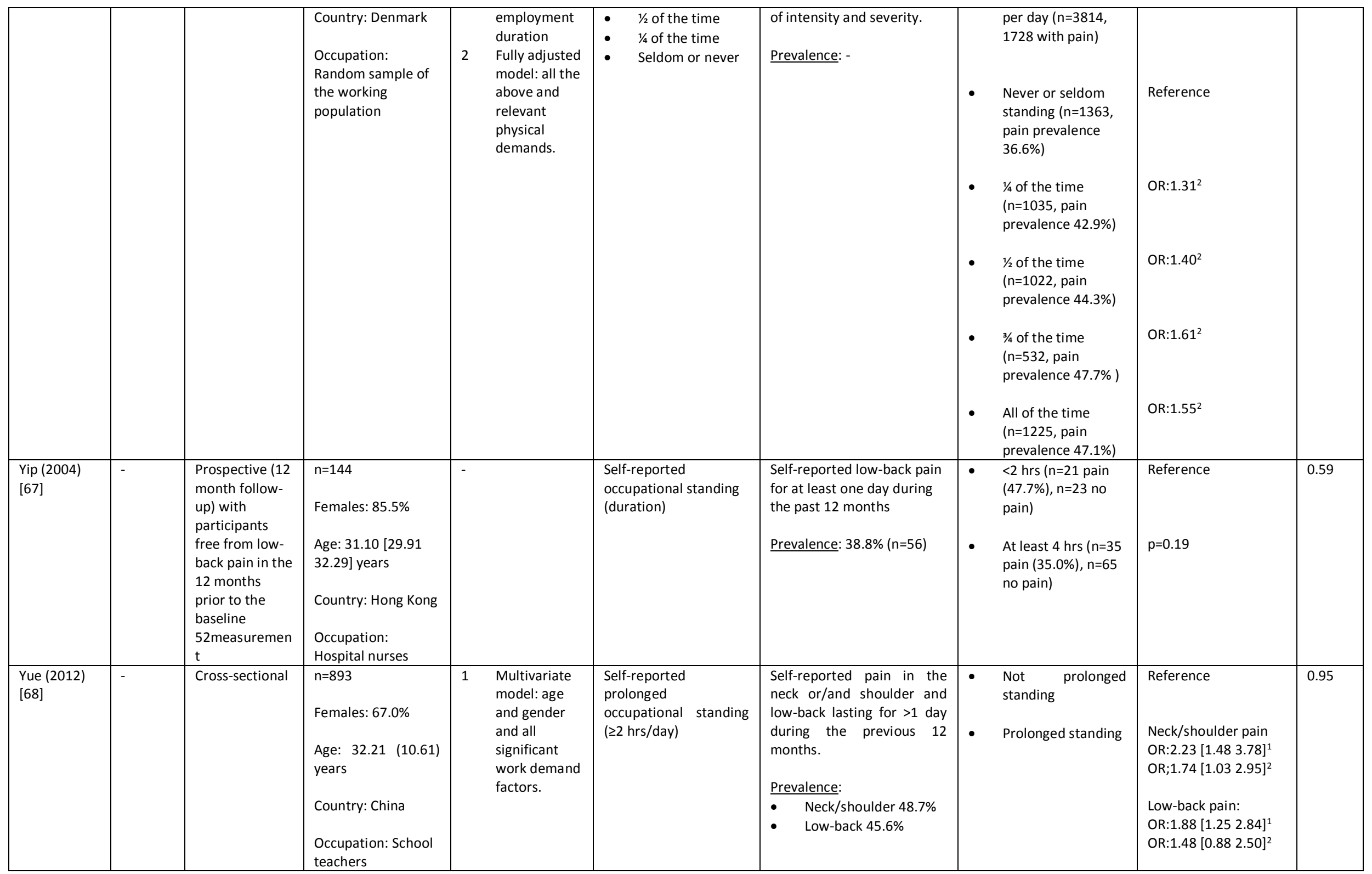


Appendix 10. Forest plot of the comparison not-excessive standing versus excessive standing on the unadjusted association with low-back symptoms. Individual study as well as pooled exposure-outcome associations are presented. Data shown for studies for which a cut-off value to distinguish not excessive standing from excessive standing of 4 hours/workday (upper panel) and 2 hours/workday (lower panel) could be adopted. $\mathrm{SE}=$ standard error; $\mathrm{Cl}$ = confidence interval; IV = inverse variance.

Odds Ratio

Odds Ratio

$\begin{array}{llll}\text { Study or Subgroup } & \log [\text { Odds Ratio] } & \text { SE Weight } \quad \text { IV, Random, } 95 \% \mathrm{Cl} & \text { IV, Random, } 95 \% \mathrm{Cl}\end{array}$

\begin{tabular}{lrlll}
\hline 2.1 .12 hours & & & & \\
Harkness 2003 & 0.09 & 0.179 & $4.3 \%$ & $1.09[0.77,1.55]$ \\
Kaneda 2001 & -0.186 & 0.052 & $5.6 \%$ & $0.83[0.75,0.92]$ \\
Li 2012 & 0.488 & 0.051 & $5.6 \%$ & $1.63[1.47,1.80]$ \\
Mehrdad 2012 & 0.538 & 0.288 & $3.0 \%$ & $1.71[0.97,3.01]$ \\
Sterud 2013 & 0.664 & 0.079 & $5.4 \%$ & $1.94[1.66,2.27]$ \\
Svensson 1983 & 0.231 & 0.157 & $4.5 \%$ & $1.26[0.93,1.71]$ \\
Svensson 1989 & 0.326 & 0.135 & $4.8 \%$ & $1.39[1.06,1.81]$ \\
Tissot 2009 & 0.273 & 0.054 & $5.6 \%$ & $1.31[1.18,1.46]$ \\
Tomita 2010 & 1.359 & 0.766 & $0.8 \%$ & $3.89[0.87,17.47]$ \\
Vahdati 2014 & -2.885 & 1.062 & $0.4 \%$ & $0.06[0.01,0.45]$ \\
Xu 1997 & 0.361 & 0.065 & $5.5 \%$ & $1.43[1.26,1.63]$ \\
Subtotal (95\% Cl) & & & $45.6 \%$ & $1.34[1.08,1.65]$ \\
\hline
\end{tabular}

Subtotal $(95 \% \mathrm{Cl})$

$45.6 \%-1.34[1.08,1.65]$

Heterogeneity: $\operatorname{Tau}^{2}=0.09 ; \mathrm{Chi}^{2}=134.96, \mathrm{df}=10(\mathrm{P} \leq 0.00001) ; \mathrm{I}^{2}=93 \%$

Test for overall effect: $Z=2.67(P=0.008)$

2.1.2 4 hours

Chandraskan 2003

Kaneda 2001

Karahan 2009

Levangie 1999

Li 2012

Mehrdad 2012

Nahit 2001

Sterud 2013

Svensson 1983

Svensson 1989

Tissot 2009

Tomita 2010

Vahdati 2014

Yip 2004

Subtotal $(95 \% \mathrm{Cl})$

$\begin{array}{rrr}0.095 & 0.194 & 4.1 \% \\ -0.186 & 0.052 & 5.6 \% \\ 0.557 & 0.13 & 4.9 \% \\ 0.013 & 0.255 & 3.4 \% \\ 0.488 & 0.051 & 5.6 \% \\ 0.538 & 0.288 & 3.0 \% \\ 0.279 & 0.178 & 4.3 \% \\ 0.648 & 0.075 & 5.4 \% \\ 0.429 & 0.176 & 4.3 \% \\ 0.346 & 0.135 & 4.8 \% \\ 0.273 & 0.054 & 5.6 \% \\ 1.359 & 0.766 & 0.8 \% \\ -2.885 & 1.062 & 0.4 \% \\ -0.528 & 0.367 & 2.3 \% \\ & & 54.4 \%\end{array}$

$1.10[0.75,1.61]$

$0.83[0.75,0.92]$

$1.75[1.35,2.25]$

$1.01[0.61,1.67]$

$1.63[1.47,1.80]$

$1.71[0.97,3.01]$

$1.32[0.93,1.87]$

$1.91[1.65,2.21]$

$1.54[1.09,2.17]$

$1.41[1.08,1.84]$

$1.31[1.18,1.46]$

$3.89[0.87,17.47]$

$0.06[0.01,0.45]$

$0.59[0.29,1.21]$

$1.31[1.06,1.61]$

Heterogeneity: $\operatorname{Tau}^{2}=0.11 ; \mathrm{Ch}^{2}=144.85, \mathrm{df}=13(\mathrm{P}<0.00001) ;\left.\right|^{2}=91 \%$

Test for overall effect: $Z=2.55$ ( $P=0.01$ )

Total $(95 \% \mathrm{Cl})$

$100.0 \% \quad 1.32[1.15,1.52]$

Heterogeneity: $\operatorname{Tau}^{2}=0.09 ; \mathrm{Chi}^{2}=279.82, \mathrm{df}=24(\mathrm{P}<0.00001) ; \mathrm{I}^{2}=91 \%$

Test for overall effect: $Z=3.90$ ( $P<0.0001$ )

Test for subqroup differences: $\mathrm{Chi}^{2}=0.02, \mathrm{df}=1(\mathrm{P}=0.88), \mathrm{I}^{2}=0 \%$

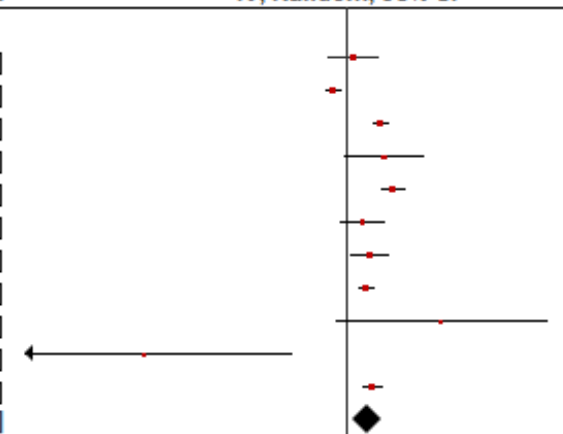


Appendix 11. Forest plot of the comparison not-excessive standing versus excessive standing (adopting a 4 hours/workday cut-off value when possible) on the association with low-back symptoms. Individual study as well as pooled exposure-outcome associations are presented. Data for studies for which unadjusted (upper panel) as well as adjusted (lower panel) associations were reported. SE = standard error; $\mathrm{Cl}=$ confidence interval; IV = inverse variance.

Odds Ratio

Odds Ratio

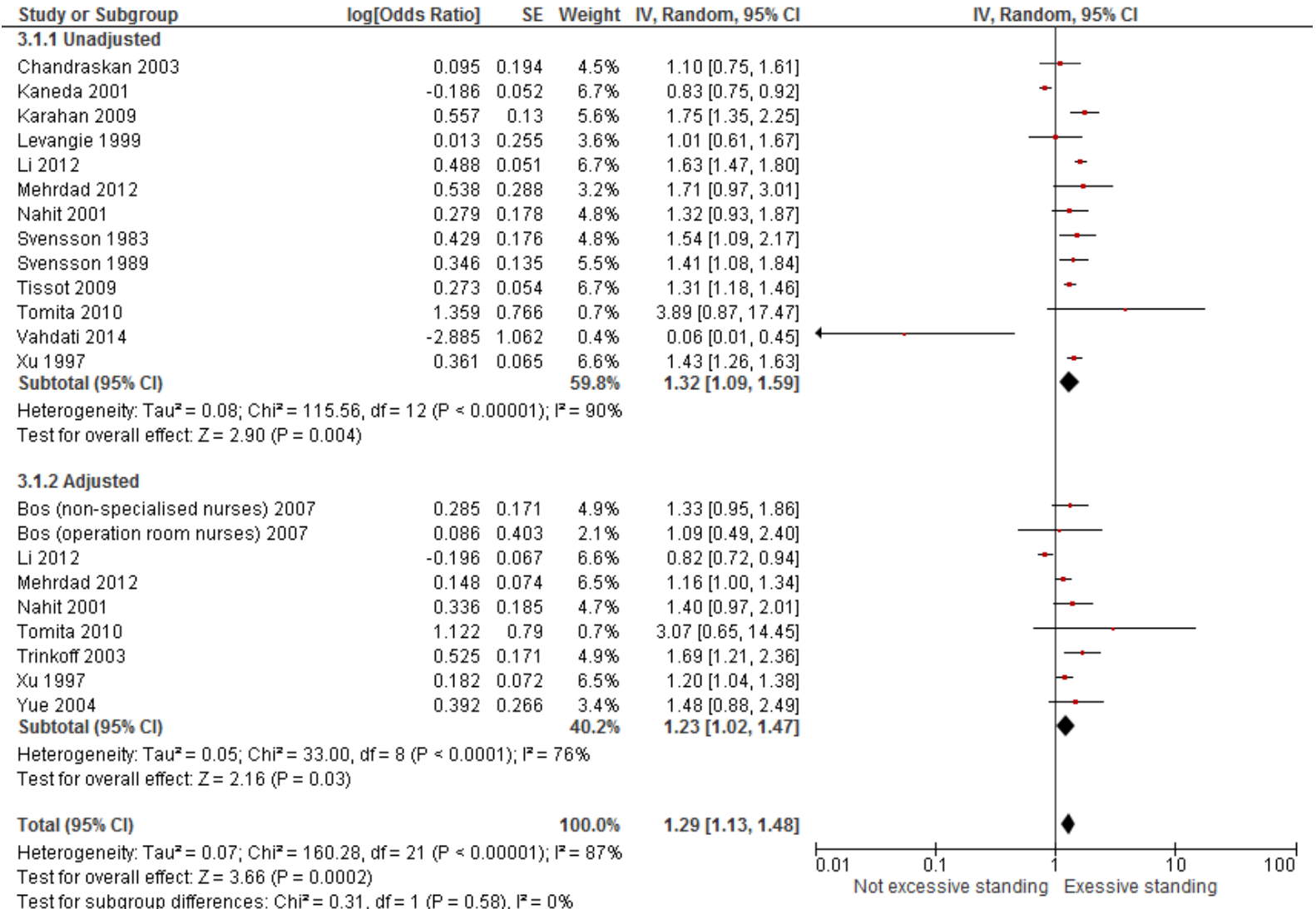


Appendix 12. Forest plot of the comparison not-excessive standing versus excessive standing (adopting a 4 hours/workday cut-off value when possible) on the unadjusted association with low-back symptoms. Individual study as well as pooled exposure-outcome associations are presented. Data for studies that reported on general occupational study samples (upper panel) as well as those which reported on specific occupational study samples (lower panel). $\mathrm{SE}=$ standard error; $\mathrm{Cl}=$ confidence interval; $\mathrm{IV}=$ inverse variance.

Odds Ratio

Odds Ratio

Study or Subgroup $\quad \log [$ Odds Ratio] $\quad$ SE Weight $\quad$ IV, Random, $95 \% \mathrm{Cl} \quad$ IV, Random, $95 \% \mathrm{Cl}$

$\begin{array}{lrrrr}\text { 4.1.1 General study population } & & & & \\ \text { Harkness 2003 } & 0.09 & 0.179 & 6.6 \% & 1.09[0.77,1.55] \\ \text { Nahit 2001 } & 0.279 & 0.178 & 6.7 \% & 1.32[0.93,1.87] \\ \text { Sterud 2013 } & 0.664 & 0.079 & 8.4 \% & 1.94[1.66,2.27] \\ \text { Svensson 1983 } & 0.429 & 0.176 & 6.7 \% & 1.54[1.09,2.17] \\ \text { Svensson 1989 } & 0.346 & 0.135 & 7.5 \% & 1.41[1.08,1.84] \\ \text { Tissot 2009 } & 0.273 & 0.054 & 8.7 \% & 1.31[1.18,1.46] \\ \text { Xu 1997 } & 0.361 & 0.065 & 8.6 \% & 1.43[1.26,1.63] \\ \text { Yip 2004 } & -0.528 & 0.367 & 3.6 \% & 0.59[0.29,1.21] \\ \text { Subtotal (95\% Cl) } & & & 56.8 \% & \mathbf{1 . 4 0}[1.20,1.62]\end{array}$

Heterogeneity: $\operatorname{Tau}^{2}=0.03 ; \mathrm{Chi}^{2}=25.90, \mathrm{df}=7(\mathrm{P}=0.0005) ; \mathrm{I}^{2}=73 \%$

Test for overall effect: $Z=4.35(P<0.0001)$

4.1.2 Specific study populaiton

$\begin{array}{lrrrr}\text { Chandraskan 2003 } & 0.095 & 0.194 & 6.3 \% & 1.10[0.75,1.61] \\ \text { Kaneda 2001 } & -0.186 & 0.052 & 8.8 \% & 0.83[0.75,0.92] \\ \text { Karahan 2009 } & 0.557 & 0.13 & 7.6 \% & 1.75[1.35,2.25] \\ \text { Levangie 1999 } & 0.013 & 0.255 & 5.2 \% & 1.01[0.61,1.67] \\ \text { Li 2012 } & 0.488 & 0.051 & 8.8 \% & 1.63[1.47,1.80] \\ \text { Mehrdad 2012 } & 0.538 & 0.288 & 4.7 \% & 1.71[0.97,3.01] \\ \text { Tomita 2010 } & 1.359 & 0.766 & 1.2 \% & 3.89[0.87,17.47] \\ \text { Vahdati 2014 } & -2.885 & 1.062 & 0.7 \% & 0.06[0.01,0.45] \\ \text { Subtotal (95\% Cl) } & & & 43.2 \% & 1.24[0.86,1.78]\end{array}$

Subtotal $(95 \% \mathrm{Cl})$

$P<0.00001) ;\left.\right|^{2}=93 \%$

Test for overall effect: $Z=1.17(P=0.24)$

Total $(95 \% \mathrm{Cl}) \quad 100.0 \% \quad 1.31[1.10,1.56]$

Heterogeneity: $\operatorname{Tau}^{2}=0.09 ; \mathrm{Chi}^{2}=147.25, \mathrm{df}=15(\mathrm{P}<0.00001) ; \mathrm{I}^{2}=90 \%$

Test for overall effect: $Z=3.00(P=0.003)$

Test for subqroup differences: $\mathrm{Ch}^{2}=0.36, \mathrm{df}=1(\mathrm{P}=0.55), \mathrm{I}^{2}=0 \%$

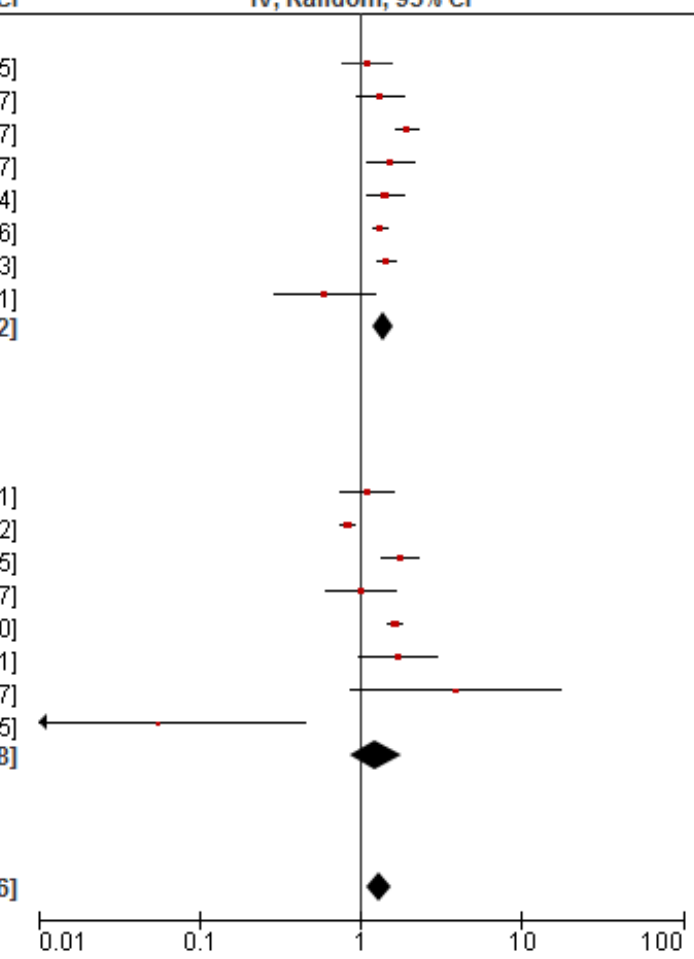

Not excessive standing Exessive standing 
Appendix 13. Forest plot of the comparison not-excessive standing versus excessive standing (adopting a 4 hours/workday cut-off value when possible) on the unadjusted association with low-back symptoms. Individual study as well as pooled exposure-outcome associations are presented. Data shown for studies that were considered of low methodological quality (upper panel) as well as high methodological quality (lower panel). SE = standard error; $\mathrm{Cl}$ = confidence interval; IV = inverse variance.

Odds Ratio

Odds Ratio

Study or Subgroup log[Odds Ratio] SE Weight IV, Random, 95\% Cl $\quad$ IV, Random, $95 \% \mathrm{C}$

$\begin{array}{lrlll}\text { 5.1.1 Low methodological quality } & & & \\ \text { Kaneda 2001 } & -0.186 & 0.052 & 8.8 \% & 0.83[0.75,0.92] \\ \text { Levangie 1999 } & 0.013 & 0.255 & 5.2 \% & 1.01[0.61,1.67] \\ \text { Li } 2012 & 0.488 & 0.051 & 8.8 \% & 1.63[1.47,1.80] \\ \text { Svensson } 1983 & 0.429 & 0.176 & 6.7 \% & 1.54[1.09,2.17] \\ \text { Svensson 1989 } & 0.346 & 0.135 & 7.5 \% & 1.41[1.08,1.84] \\ \text { Subtotal (95\% Cl) } & & & 36.9 \% & \mathbf{1 . 2 5}[0.85,1.82]\end{array}$

Heterogeneity: $\mathrm{Tau}^{2}=0.17 ; \mathrm{Chi}^{2}=89.95, \mathrm{df}=4(\mathrm{P}<0.00001) ; \mathrm{I}^{2}=96 \%$

Test for overall effect: $Z=1.14(P=0.25)$

5.1.2 High methodological quality

$\begin{array}{lrrrr}\text { Chandraskan 2003 } & 0.095 & 0.194 & 6.3 \% & 1.10[0.75,1.61] \\ \text { Harkness 2003 } & 0.09 & 0.179 & 6.6 \% & 1.09[0.77,1.55] \\ \text { Karahan 2009 } & 0.557 & 0.13 & 7.6 \% & 1.75[1.35,2.25] \\ \text { Mehrdad 2012 } & 0.538 & 0.288 & 4.7 \% & 1.71[0.97,3.01] \\ \text { Nahit 2001 } & 0.279 & 0.178 & 6.7 \% & 1.32[0.93,1.87] \\ \text { Sterud 2013 } & 0.664 & 0.079 & 8.4 \% & 1.94[1.66,2.27] \\ \text { Tissot 2009 } & 0.273 & 0.054 & 8.7 \% & 1.31[1.18,1.46] \\ \text { Tomita 2010 } & 1.359 & 0.766 & 1.2 \% & 3.89[0.87,17.47] \\ \text { Vahdati 2014 } & -2.885 & 1.062 & 0.7 \% & 0.06[0.01,0.45] \\ \text { Xu 1997 } & 0.361 & 0.065 & 8.6 \% & 1.43[1.26,1.63] \\ \text { Yip 2004 } & -0.528 & 0.367 & 3.6 \% & 0.59[0.29,1.21] \\ \text { Subtotal (95\% Cl) } & & & 63.1 \% & 1.38[1.16,1.64]\end{array}$

Heterogeneity: Tau $^{2}=0.05 ; \mathrm{Chi}^{2}=41.27, \mathrm{df}=10(\mathrm{P}<0.0001) ; \mathrm{I}^{2}=76 \%$

Test for overall effect: $Z=3.64(P=0.0003)$

Total $(95 \% \mathrm{Cl})$

$100.0 \% \quad 1.31[1.10,1.56]$

Heterogeneity: Tau $^{2}=0.09 ; \mathrm{Chi}^{2}=147.25, \mathrm{df}=15(\mathrm{P} \leq 0.00001) ; \mathrm{I}^{2}=90 \%$

Test for overall effect: $Z=3.00(P=0.003)$

Test for subqroup differences: $\mathrm{Chi}^{2}=0.22, \mathrm{df}=1(\mathrm{P}=0.64), \mathrm{I}^{2}=0 \%$

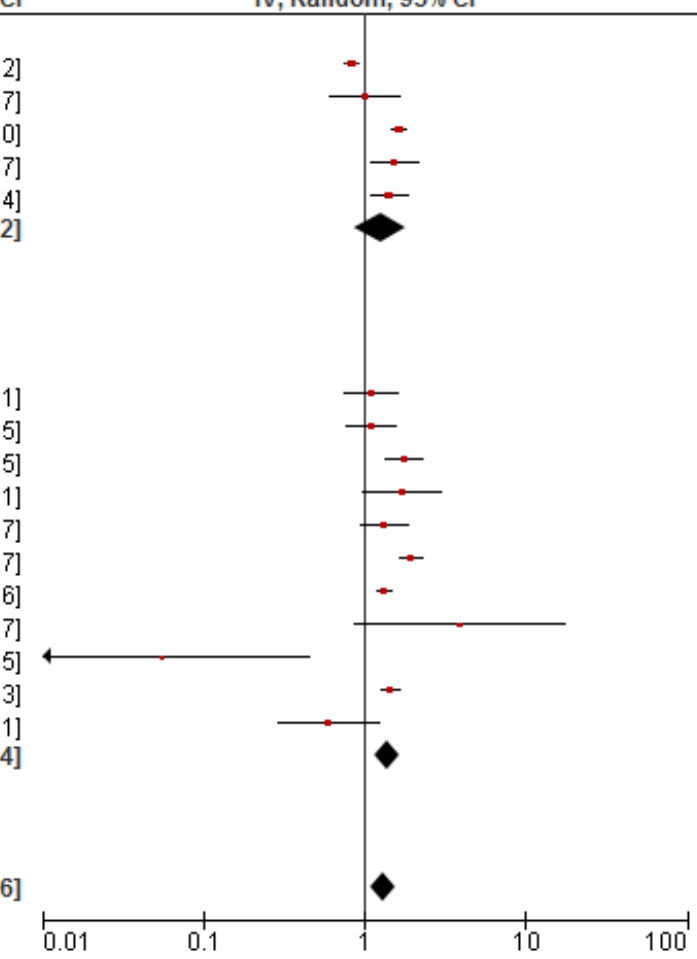

Not excessive standing Exessive standing 
Appendix 14. Funnel plot for the association of excessive standing and low-back symptoms. Dots represent (with circles being crosssectional study designs and diamonds being longitudinal study designs) the individual study estimates while the vertical line depicts the summary effect of the exposure-outcome associations.

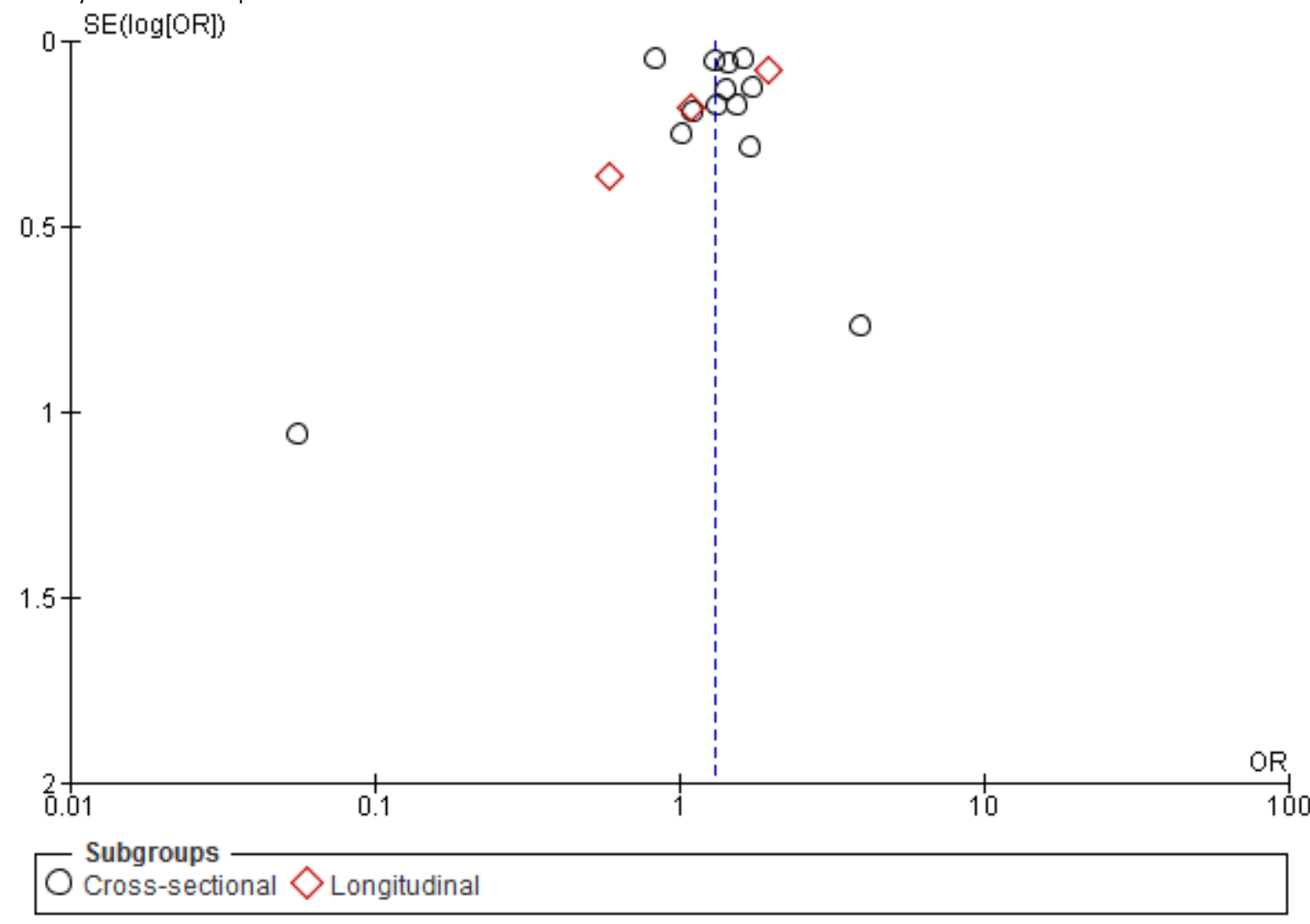




\section{Reference}

1. Safe Work Australia. National hazard exposure worker surveillance: exposure to biomechanical demands, pain and fatigue symptoms and the provision of controlls in Australian workplaces. Canberra, Australia, 2011.

2. Tissot F, Messing K, Stock S. Standing, sitting and associated working conditions in the Quebec population in 1998. Ergonomics 2005;48(3):249-69

3. Munch Nielsen C, Nidhi G, Knudsen LE, Holtermann A. Association of objectively measured occupational walking and standing still with low back pain: a cross-sectional study. Ergonomics 2016;11:1-20

4. Tremblay MS, Colley RC, Saunders TJ, Healy GN, Owen N. Physiological and health implications of a sedentary lifestyle. Appl Physiol Nutr Metab 2010;35(6):725-40

5. Thorp AA, Owen N, Neuhaus M, Dunstan DW. Sedentary behaviors and subsequent health outcomes in adults a systematic review of longitudinal studies, 1996-2011. Am J Prev Med 2011;41(2):207-15

6. Straker L, Coenen P, Dunstan DW, Gilson N, Healy GN. Sedentary work - Evidence on an emergent work health and safety issue. In: Safe Work Australia, ed. Canberra, Australia, 2016.

7. Shrestha N, ljaz S, Kukkonen-Harjula KT, Kumar S, Nwankwo CP. Workplace interventions for reducing sitting at work. Cochrane Database Syst Rev 2015;1:CD010912

8. Buckley JP, Hedge A, Yates T, et al. The sedentary office: a growing case for change towards better health and productivity. Expert statement commissioned by Public Health England and the Active Working Community Interest Company. Br J Sports Med 2015;10.1136/bjsports-2015094618

9. Pronk NP, Katz AS, Lowry M, Payfer JR. Reducing occupational sitting time and improving worker health: the Take-a-Stand Project, 2011. Prev Chronic Dis 2012;9:E154

10. Neuhaus M, Eakin EG, Straker L, et al. Reducing occupational sedentary time: a systematic review and meta-analysis of evidence on activity-permissive workstations. Obes Rev 2014;15(10):822-38

11. Callaghan JP, de Carvalho D, Gallagher K, Karakolis T, Nelson-Wong E. Is standing the solution to sedentary office work? Ergon Des 2015;23(3):20-24

12. Waters TR, Dick RB. Evidence of health risks associated with prolonged standing at work and intervention effectiveness. Rehabil Nurs 2014;40(3):148-65

13. Jawien $A$. The influence of environmental factors in chronic venous insufficiency. Angiology 2003;54 (Suppl 1):S19-S31

14. Beebe-Dimmer JL, Pfeifer JR, Engle JS, Schottenfeld D. The epidemiology of chronic venous insufficiency and varicose veins. Ann Epidemiol 2005;15(3):175-84

15. Bonzini M, Coggon D, Palmer KT. Risk of prematurity, low birthweight and pre-eclampsia in relation to working hours and physical activities: a systematic review. Occup Environ Med 2007;64(4):228-43

16. da Costa BR, Vieira ER. Risk factors for work-related musculoskeletal disorders: A systematic review of recent longitudinal studies. Am J Ind Med 2010;53(3):285-323

17. Andersen JH, Haahr JP, Frost P. Risk factors for more severe regional musculoskeletal symptoms: a two-year prospective study of a general working population. Arthritis Rheum 2007;56(4):1355-64

18. Kmet LM, Lee RC, Cook LS. Standard quality assessment criteria for evaluating primary research papers from a variety of fields In: Alberta Heritage Foundation for Medical Research, ed. Edmonton, Canada, 2004.

19. Griffith LE, Hogg-Johnson S, Cole DC, et al. Low-back pain definitions in occupational studies were categorized for a meta-analysis using Delphi consensus methods. J Clin Epidemiol 2007;60(6):625-33

20. Abd Rahman MN, Aziz FA, Yusuff RM. Survey of body part symptoms among workers in a car tyre service centre. J Hum Ergol 2010;39(1):53-56 
21. Babiolakis CS, Kuk JL, Drake JD. Differences in lumbopelvic control and occupational behaviours in female nurses with and without a recent history of low back pain due to back injury. Ergonomics 2015;58(2):235-45

22. Barghout NH, Al-Habashneh R, AL-Omiri MK. Risk factors and prevalence of musculoskeletal disorders among jordanian dentists. Jordan Med J 2011;45(2):195-204

23. Bejia I, Younes M, Jamila HB, et al. Prevalence and factors associated to low back pain among hospital staff. Joint Bone Spine

2005;72(3):254-59

24. Bener A, Al-Suwaidi J, Al-Jaber K, Al-Marri S, Elbagi IE. Epidemiology of hypertension and its associated risk factors in the Qatari population. J Hum Hypertens 2004;18(7):529-30

25. Bener A, Dafeeah EE, Alnaqbi K, et al. An epidemiologic analysis of low back pain in primary care: a hot humid country and global comparison. J Prim Care Community Health 2013;4(3):220-27

26. Bergquist-Ullman M, Larsson U. Acute low back pain in industry. A controlled prospective study with special reference to therapy and confounding factors. Acta Orthop Scand 1977;170:1117

27. Bos E, Krol B, van der Star L, Groothoff J. Risk factors and musculoskeletal complaints in nonspecialized nurses, IC nurses, operation room nurses, and X-ray technologists. Int Arch Occup Environ Health 2007;80(3):198-206

28. Chandrasakaran A, Chee HL, Rampal KG, Tan GL. The prevalence of musculoskeletal problems and risk factors among women assembly workers in the semiconductor industry. Med J Malaysia 2003;58(5):657-66

29. da Silva MC, Fassa AG, Kriebel D. Musculoskeletal pain in ragpickers in a southern city in Brazil. Am J Ind Med 2006;49(5):327-36

30. Duquette J, Lortie M, Rossignol M. Perception of difficulties for the back related to assembly work: general findings and impact of back health. Appl Ergon 1997;28(5-6):389-96

31. Engels JA, van der Gulden JW, Senden TF, van't Hof B. Work related risk factors for musculoskeletal complaints in the nursing profession: results of a questionnaire survey. Occup Environ Med 1996;53(9):636-41

32. Hallman DM, Ekman AH, Lyskov E. Changes in physical activity and heart rate variability in chronic neck-shoulder pain: monitoring during work and leisure time. Int Arch Occup Environ Health 2014;87(7):735-44

33. Harkness EFM, Macfarlane GJ, Nahit ES, Silman AJ, McBeth J. Risk factors for new-onset low back pain amongst cohorts of newly employed workers. Rheumatology 2003;42(8):959-68

34. Hill JJ, 3rd, Slade MD, Russi MB. Anthropometric measurements, job strain, and prevalence of musculoskeletal symptoms in female medical sonographers. Work 2009;33(2):181-89

35. Hou JY, Shiao JS. Risk factors for musculoskeletal discomfort in nurses. J Nurs Res 2006;14(3):22836

36. Jellad A, Lajili $H$, Boudokhane $S$, Migaou $H$, Maatallah S, Frih ZBS. Musculoskeletal disorders among Tunisian hospital staff: Prevalence and risk factors. The Egyptian Rheumatologist 2013;35(2):59-63

37. Jones GT, Harkness EF, Nahit ES, McBeth J, Silman AJ, Macfarlane GJ. Predicting the onset of knee pain: results from a 2-year prospective study of new workers. Ann Rheum Dis 2007;66(3):400-06

38. Kaneda K, Shirai Y, Miyamoto M. An epidemiological study on occupational low back pain among people who work in construction. J Nippon Med Sch 2001;68(4):310-17

39. Karahan A, Kav S, Abbasoglu A, Dogan N. Low back pain: prevalence and associated risk factors among hospital staff. J Adv Nurs 2009;65(3):516-24

40. Külcü DG, Gülşen G, Altunok TC, Küçükoğlu D, Naderi S. Neck and low back pain among dentistry staff. 2010;25(3):122-29

41. Lehto TU, Helenius HY, Alaranta HT. Musculoskeletal symptoms of dentists assessed by a multidisciplinary approach. Community Dent Oral Epidemiol 1991;19(1):38-44 
42. Leino $T$, Kähkönen $E$, Saarinen $L$, Henriks-Eckerman $M L$, Paakkulainen $H$. Working conditions and health in hairdressing salons. Appl Occup Environ Hyg 1999;14(1):26-33

43. Leroux I, Dionne CE, Bourbonnais R, Brisson C. Prevalence of musculoskeletal pain and associated factors in the Quebec working population. Int Arch Occup Environ Health 2005;78(5):379-86

44. Levangie PK. Association of low back pain with self-reported risk factors among patients seeking physical therapy services. Phys Ther 1999;79(8):757-66

45. Li JY, Wang S, He LH, et al. Risk factors of low back pain among the Chinese occupational population: a case-control study. Biomed Environ Sci 2012;25(4):421-29

46. Mehrdad R, Dennerlein JT, Morshedizadeh M. Musculoskeletal disorders and ergonomic hazards among Iranian physicians. Arch Iran Med 2012;15(6):370-74

47. Messing K, Kilbom A. Standing and very slow walking: foot pain-pressure threshold, subjective pain experience and work activity. Appl Ergon 2001;32(1):81-90

48. Messing K, Tissot F, Stock S. Distal lower-extremity pain and work postures in the Quebec population. Am J Public Health 2008;98(4):705-13

49. Mohseni-Bandpei MA, Ahmad-Shirvani M, Golbabaei N, Behtash H, Shahinfar Z, Fernández-delas-Peñas C. Prevalence and risk factors associated with low back pain in Iranian surgeons. J Manipulative Physiol Ther 2011;34(6):362-70

50. Nahit ES, Macfarlane GJ, Pritchard CM, Cherry NM, Silman AJ. Short term influence of mechanical factors on regional musculoskeletal pain: a study of new workers from 12 occupational groups. Occup Environ Med 2001;58(6):374-81

51. Phajan T, Nilvarangkul K, Settheetham D, Laohasiriwong W. Work-related musculoskeletal disorders among sugarcane farmers in north-eastern Thailand. Asia Pac J Public Health 2014;26(3):320-27

52. Pope DP, Hunt IM, Birrell FN, Silman AJ, Macfarlane GJ. Hip pain onset in relation to cumulative workplace and leisure time mechanical load: a population based case-control study. Ann Rheum Dis 2003;62(4):322-26

53. Rodigari A, Bejor M, Carlisi E, Lisi C, Tinelli C, Toffola ED. Identification of risk factors for fatigue and pain when performing surgical interventions. G Ital Med Lav Ergon 2012;34(4):432-37

54. Samad NIA, Abdullah H, Moin S, Tamrin SBM, Hashim Z. Prevalence of low back pain and its risk factors among school teachers. Am J Appl Sci 2010;7(5):634-39

55. Sanya AO, Ogwumike OO. Low back pain prevalence amongst industrial workers in the private sector in Oyo State, Nigeria. Afr J Med Med Sci 2005;34(3):245-49

56. Schierhout GH, Meyers JE, Bridger RS. Work related musculoskeletal disorders and ergonomic stressors in the South African workforce. Occup Environ Med 1995;52(1):46-50

57. Smith DR, Takeda Y, Mizutani T, Yamagata Z. Musculoskeletal disorders and skin disease among workers in a Japanese CD manufacturing plant. J UOEH 2002;24(4):397-404

58. Sterud T, Tynes T. Work-related psychosocial and mechanical risk factors for low back pain: a 3year follow-up study of the general working population in Norway. Occup Environ Med 2013;70(5):296-302

59. Svensson HO, Andersson GB. Low-back pain in 40- to 47-year-old men: work history and work environment factors. Spine 1983;8(3):272-6

60. Svensson HO, Andersson GB. The relationship of low-back pain, work history, work environment, and stress. A retrospective cross-sectional study of 38 - to 64-year-old women. Spine 1989;14(5):517-22

61. Tissot F, Messing K, Stock S. Studying the relationship between low back pain and working postures among those who stand and those who sit most of the working day. Ergonomics 2009;52(11):1402-18

62. Tomita S, Arphorn S, Muto T, Koetkhlai K, Naing SS, Chaikittiporn C. Prevalence and risk factors of low back pain among Thai and Myanmar migrant seafood processing factory workers in Samut Sakorn Province, Thailand. Ind Health 2010;48(3):283-91

63. Trinkoff AM, Lipscomb JA, Geiger-Brown J, Storr CL, Brady BA. Perceived physical demands and reported musculoskeletal problems in registered nurses. Am J Prev Med 2003;24(3):270-75 
64. Vahdati SS, Khiavi RS, Ghafouri RR, Adimi I. Evaluation of prevalence of low back pain among residents of tabriz university of medical sciences in relation with their position in work. Turk J Emerg Med 2014;14(3):125-29

65. Xiao H, McCurdy SA, Stoecklin-Marois MT, Li CS, Schenker MB. Agricultural work and chronic musculoskeletal pain among Latino farm workers: the MICASA study. Am J Ind Med 2013;56(2):216-25

66. Xu Y, Bach E, Orhede E. Work environment and low back pain: the influence of occupational activities. Occup Environ Med 1997;54(10):741-45

67. Yip VY. New low back pain in nurses: work activities, work stress and sedentary lifestyle. J Adv Nurs 2004;46(4):430-40

68. Yue P, Liu F, Li L. Neck/shoulder pain and low back pain among school teachers in China, prevalence and risk factors. BMC Public Health 2012;12:789

69. Antle DM, Cote JN. Relationships between lower limb and trunk discomfort and vascular, muscular and kinetic outcomes during stationary standing work. Gait Posture 2013;37(4):615-9

70. Gallagher KM, Nelson-Wong E, Callaghan JP. Do individuals who develop transient low back pain exhibit different postural changes than non-pain developers during prolonged standing? Gait Posture 2011;34(4):490-95

71. Gregory DE, Callaghan JP. Prolonged standing as a precursor for the development of low back discomfort: an investigation of possible mechanisms. Gait Posture 2008;28(1):86-92

72. Balasubramanian V, Adalarasu K, Regulapati R. Comparing dynamic and stationary standing postures in an assembly task. Int J Ind Ergonom 2009;39(5):649-54

73. Chester MR, Rys MJ, Konz SA. Leg swelling, comfort and fatigue when sitting, standing, and sit/standing. Int J Ind Ergonom 2002;29(5):289-96

74. Verbunt JA, Seelen HA, Vlaeyen JW, et al. Disuse and deconditioning in chronic low back pain: concepts and hypotheses on contributing mechanisms. Eur J Pain 2003;7(1):9-21

75. Mathiassen SE. Diversity and variation in biomechanical exposure: what is it, and why would we like to know? Appl Ergon 2006;37(4):419-27

76. Kwak L, Proper KI, Hagstromer M, Sjostrom M. The repeatability and validity of questionnaires assessing occupational physical activity--a systematic review. Scand J Work Environ Health 2011;37(1):6-29

77. Coenen P, Kingma I, Boot CRL, Twisk JWR, Bongers PM, van Dieën JH. Cumulative low back load at work as a risk factor of low back pain: a prospective cohort study. J Occup Rehabil 2013;23(1):11-18

78. Coenen P, Mathiassen SE, Kingma I, Boot CR, Bongers PM, Dieën van JH. The effect of the presence and characteristics of an outlying group on exposure-outcome associations. Scand J Work Environ Health 2015;41(1):65-74

79. Hartvigsen J, Bakketeig LS, Leboeuf-Yde C, Engberg M, Lauritzen T. The association between physical workload and low back pain clouded by the "healthy worker" effect: populationbased cross-sectional and 5-year prospective questionnaire study. Spine 2001;26(16):178892 